\title{
Shedding new light on the treatment of basal cell carcinoma
}

Citation for published version (APA):

Kuijpers, D. I. M. (2007). Shedding new light on the treatment of basal cell carcinoma. [Doctoral Thesis, Maastricht University]. Datawyse / Universitaire Pers Maastricht. https://doi.org/10.26481/dis.20070316dk

Document status and date:

Published: 01/01/2007

DOI:

$10.26481 /$ dis.20070316dk

Document Version:

Publisher's PDF, also known as Version of record

\section{Please check the document version of this publication:}

- A submitted manuscript is the version of the article upon submission and before peer-review. There can be important differences between the submitted version and the official published version of record.

People interested in the research are advised to contact the author for the final version of the publication, or visit the DOI to the publisher's website.

- The final author version and the galley proof are versions of the publication after peer review.

- The final published version features the final layout of the paper including the volume, issue and page numbers.

Link to publication

\footnotetext{
General rights rights.

- You may freely distribute the URL identifying the publication in the public portal. please follow below link for the End User Agreement:

www.umlib.nl/taverne-license

Take down policy

If you believe that this document breaches copyright please contact us at:

repository@maastrichtuniversity.nl

providing details and we will investigate your claim.
}

Copyright and moral rights for the publications made accessible in the public portal are retained by the authors and/or other copyright owners and it is a condition of accessing publications that users recognise and abide by the legal requirements associated with these

- Users may download and print one copy of any publication from the public portal for the purpose of private study or research.

- You may not further distribute the material or use it for any profit-making activity or commercial gain

If the publication is distributed under the terms of Article $25 \mathrm{fa}$ of the Dutch Copyright Act, indicated by the "Taverne" license above, 
Shedding new light on the treatment of basal cell carcinoma 
(C) D.I.M. Kuijpers, Maastricht 2007 ISBN 978-90-5278-601-8

Grafische vormgeving:

Brit Zweegers

Druk:

Datawyse Maastricht 


\title{
Shedding new light on the treatment of basal cell carcinoma
}

\author{
Proefschrift \\ ter verkrijging van de graad van doctor \\ aan de universiteit Maastricht \\ op gezag van de rector magnificus, \\ Prof. mr. G.P.M.F. Mols \\ volgens het besluit van het college van Decanen, \\ in het openbaar te verdedigen op
}

16 maart 2007 om 14.00 uur

door

Daniëlle Irene Maria Kuijpers

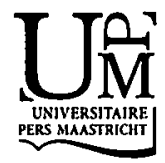




\section{Promotores}

Prof. dr. H.A.M. Neumann

Prof. dr. P.M. Steijlen

\section{Copromotor}

Dr. M.R.T.M. Thissen

\section{Beoordelingscommissie}

Prof. dr. J.J. Manni (voorzitter)

Dr. J.M.A. de Jong

Prof. dr. P.C.M. vd Kerkhof (Radboud University Nijmegen)

Prof. dr. A.M. Wennberg (Göteborg University, Sweden)

This thesis was generously supported by:

3M Pharma, Galderma SA, Waldmann Medische Techniek BV and by: Sara Lee Household \& Bodycare BV, Sigma Tau BV, Bosman medische en orthopedische hulpmiddelen, Eurotec BV, Laservision Instruments BV, Astellas Pharma BV, Bauerfind Benelux BV, BSN Medical BV, Coloplast BV, GlaxoSmithKline BV, Leo Pharma BV, Novartis Pharma BV, Pie Medical Benelux BV, Serono Benelux BV, Vichy Cosmetique Active, Fagron Pharmaceuticals BV, Smith \& Nephew, Wyeth Pharmaceuticals BV 
Aan mijn ouders, Mark en Teun 


\section{Contents}

List of abbreviations

General introduction and aims of the thesis

Chapter 1 Review on treatments of basal cell carcinoma

\section{Chapter 2 The cutting edge}

2.1 Surgical excision versus curettage plus cryosurgery in the treatment of basal cell carcinoma of the head and neck.

2.2 Mohs' micrographic surgery for treatment of basal cell carcinoma of the face; review of the literature and results of a retrospective study.

2.3 Imiquimod $5 \%$ cream and photodynamic therapy as adjuvant treatment after incomplete Mohs' micrographic surgery for large, mixed type basal cell carcinoma.

2.4 Does systemic azithomycin inmprove the survival of a full thickness graft on the nose?

\section{Chapter 3 Photodynamic therapy enlightened}

3.1 Comparison of fractionated photodynamic therapy with surgical excision in the treatment of nodular basal cell carcinoma

3.2 Different pain sensations in photodynamic therapy of 95 nodular basal cell carcinoma. Results from a prospective trial and a review of the literature.

3.3 Similar effectiveness of methyl aminolevulinate and 106 5-aminolevulinate in topical photodynamic therapy for nodular basal cell carcinoma.

\section{Chapter 4 Summary and general discussion}

Bibliography

Samenvatting

Dankwoord 


\section{List of abbreviations}

AK actinic keratosis, solar keratosis

ALA 5-aminolevulinic acid

$\mathrm{BCC}$ basal cell carcinoma

BCNS basal cell nevus syndrome, Gorlin's syndrome, NBCCS

CE curettage and electrodessication

CS cryosurgery, C\&C

C\&C curettage and cryosurgery, CS

$\mathrm{cm}^{2} \quad$ square centimetres

FTG full thickness graft

Gy Gray, absorbed dose of 1 Joule/kilogram

HR hazard ratio

IFN interferon

IL interleukins

$\mathrm{J}$ Joules

kV kilo Volt, applied for photon radiation

$\mathrm{keV} \quad$ kilo electron Volt, applied for electron radiation

MAL methyl ester aminolevulinic acid

MV mega Volt, applied for photon radiation

$\mathrm{MeV}$ mega electron Volt, applied for electron radiation

$\mathrm{mW} \quad$ milliwatt

MMS Mohs' micrographic surgery

$\mathrm{nBCC}$ nodular basal cell carcinoma

NMSC non-melanoma skin cancer

PDD photodynamic diagnosis

PDT photodynamic therapy

PpIX protoporphyrin IX

RCT randomised controlled trial

RT radiotherapy

SBCC superficial basal cell carcinoma

SCC squamous cell carcinoma

SE surgical excision

TLR toll like receptor

TNF tumour necrosis factor

XP Xeroderma Pigmentosa 


\section{General introduction and aims of the thesis}




\section{Incidence}

Basal cell carcinoma (BCC), first described by Jacob in 1827, is the most common malignant neoplasm in humans, and also the most common skin malignancy. ${ }^{1}$

The incidence is rising, but because adequate registration of BCC is lacking in many countries, exact, up-to-date incidence numbers are not available. However, it has been estimated that one in every 5-6 Caucasians in Europe and USA will be affected. ${ }^{2}$ In the south-eastern parts of the Netherlands, where registration does exist, BCC is calculated to account for as much as $17 \%$ of all cancer cases. ${ }^{3}$

In European countries where BCC is registered, incidence rates in the past two decades vary between 45 and 128 per 100000 person-years. ${ }^{3}$ The predicted annual incidence in the Netherlands for 2015, based on trend modelling of observed rates, is approximately 120 first primary BCCs per 100000 person-years, which represents an increase of $78 \%$ in 15 years. ${ }^{4}$ These calculations are probably an underestimation, as the registration only records the tumours that have been confirmed histologically. To illustrate this discrepancy; in the year 2000 approximately 15000 new BCCs were diagnosed in the Netherlands, calculated by extrapolation of figures from the southern part of the Netherlands, which contrasts with $30000 \mathrm{BCCs}$ as calculated from a questionnaire conducted among 293 dermatologists in the Netherlands. ${ }^{4.5}$ Clinical experts in the field of non-melanoma skin cancer (NMSC) in the Netheriands give an estimation of 80000 new BCCs per year around the year 2015. The rise in incidence is observed in both sexes, but is greatest in young female patients with $3.9 \%$ per year vs. $2.4 \%$ per year in males, and proportionally largest on the trunk, due to changing sun exposure habits. ${ }^{3,6,7}$

The incidence rates of $\mathrm{BCC}$ for those younger than 60 years have stabilised in Australia, due to intensive skin cancer prevention programmes. ${ }^{8} \mathrm{It}$ is likely that this trend may also be seen in Europe and the USA in future.

\section{Pathogenesis and risk factors}

The most common factor involved in the pathogenesis of BCC is ultraviolet (UV) light (sunlight, tanning beds, PUVA therapy). Patients with fair skin phenotypes (blue eyes, red or blond hair and easy freckling) are, as expected, particularly predisposed. ${ }^{9.10}$ The protective role of skin pigmentation is well demonstrated in coloured individuals with albinism." UV induced epidermal DNA damage is thought to be the primary carcinogenic event occurring in the development of BCC. ${ }^{12}$ Early as well as cumulative UV radiation plays an important role. UV radiation induces DNA damage because the wavelengths of the UVB radiation correspond with the absorption spectrum of DNA. The skin is able to repair this damage. However, the capacity for DNA repair declines with age and is found to be reduced in young patients with $\mathrm{BCC} .{ }^{13}$

Cumulative lifetime sun exposure is believed to increase the risk for $\mathrm{BCC}$, although other 
studies suggest that intermittent intense sun exposure early in life enhances the risk. 14,15 The use of tanning beds also contributes to the incidence of $\mathrm{BCC} .{ }^{16}$ Epidemiological studies suggest that sun exposure up to age 20 , and in particular experiencing sunburn before age 15, initiates a process of carcinogenesis that is manifested $40-60$ years later. ${ }^{10.17}$ Twenty percent of BCCs however, occur on skin that is not exposed to the sun, suggesting that other aetiological factors play a role. ${ }^{18}$ Besides causing mutations in DNA, UV radiation also contributes to carcinogenesis in general by suppressing the systemic immune responses to mutant cells through the production of suppressive cytokines. ${ }^{19}$

The complex process of carcinogenesis also involves activation of oncogenes or inactivation of tumour suppressor genes which can be regarded as acquired genetic alterations.

The most commonly acquired aberrations in skin cancer are found in the p53 (tumour suppressor) gene. In normal skin, p53 is already detectable after a single UV radiation. When the content of $p 53$ is low, cells will go into cell arrest to allow DNA repair whereas high levels of $\mathrm{p} 53$ will cause cells to progress into apoptosis. Chronic sun exposure is held responsible for the p53 mutations. P53 mutations have been found in half of all BCCs, with a strong association with aggressive BCCs. ${ }^{20}$ Adequate use of sunscreens leads to a lower level of p53 expression. ${ }^{21}$ It is suggested that p53 mutations in BCC are secondary events, occurring after tumour initiation, with an unclear contribution to tumorigenesis. ${ }^{22}$

$\mathrm{Bcl}-2$ is an important oncogene that suppresses programmed cell death (apoptosis). There are high levels of $\mathrm{Bcl}-2$ found in BCCs. This suggests a neoplastic transformation caused by extended cell survival rather than increased cell proliferation. ${ }^{23}$ Also, a dysfunction of the tumour suppressor gene p53 contributes to the development of $B C C$ by losing its normal protective function of driving damaged cells into apoptosis. ${ }^{24}$ In general, the balance between apoptosis and proliferation regulates the growth of a tumour. Proliferation studies suggest that BCC should grow much faster than it actually does, so cell loss plays an important role in the slow growth rate of $\mathrm{BCC} .^{22}$

An increased risk of $\mathrm{BCC}$ and other skin cancers has been found in organ transplant recipients, suggesting a role of immunosuppression in the pathogenesis of $\mathrm{BCC}^{25}$ The increased risk in immune-compromised patients is suggested to be due to impairment of the immune surveillance of oncogenic viruses. However, the impact of a depressed immune system in the development of BCC has been questioned. ${ }^{22}$ The major histocompatibility complex genes play an important role in controlling immune responses. HLA-DR is one of the classes of these genes. The involvement of HLA genes in the development of skin cancer was suggested as the presence of HLA-DR7 and a decrease of HLA-DR4 was found to be associated with $\mathrm{BCC}{ }^{26}$

An association has been suggested between infection with oncogenic types of human papilloma virus (HPV) and the development of BCC by promoting cell division with a reduction in DNA repair and an abrogation of UV-induced apoptosis. ${ }^{27}$ 
A causal relation has not yet been established. ${ }^{28}$ Additional risk factors that have been recognised are exposure to arsenic, coal tar derivates, nitrogen mustard, non-diagnostic $X$-ray treatment and chronic graft versus host disease. ${ }^{29-31}$ Furthermore, BCC may arise in scars, vaccination sites, ulcers, burn sites, sites of chronic inflammation and in nevus sebaceous. ${ }^{32-35}$

Finally, patients who have had one BCC, have a significantly higher risk of developing new primary lesions. ${ }^{36}$

\section{Genetic predisposition}

Several genodermatoses are known to have an enhanced risk of developing BCC. These are Basal Cell Nevus syndrome (BCNS), Xeroderma Pigmentosa (XP), Rasmussen syndrome, Rombo syndrome, Bazex syndrome, albinism and Darier's disease. ${ }^{37} \mathrm{XP}$ has served as a model to investigate whether the lack of ability to repair UV light-induced DNA damage in these patients which can lead to the development of NMSC at early age, is also valid for non-XP patients.

Studies in patients with BCNS suggest that a mutation in a tumour suppressor gene, the 'patched gene' (PTCH), may play a role in the development of BCCs in these patients. PTCH may also play a role in the pathogenesis in patients with sporadic BCCs. ${ }^{38}$ This gene is located on the long arm of chromosome 9 (9q22). Its allelic loss or inactivation is associated with both sporadic and $\mathrm{BCNS}$ associated $\mathrm{BCC}$. The $\mathrm{PTCH}$ gene product is part of a receptor for a protein called Sonic Hedgehog, which is part of a complex regulatory network that is essential for hair follicle morphogenesis. It is suggested that the development in the context of PTCH mutations may represent uncontrolled hair follicle morphogenesis. ${ }^{22}$

In addition to mutations in PTCH, which occur in $30-40 \%$ of sporadic BCCs, $20 \%$ of BCCs show mutations in 'smoothened' (SMOH), which is another protein in the hedgehog signalling pathway. ${ }^{39,40}$

Proteins that mediate detoxification processes in the skin may also be involved in susceptibility for BCC. For example, glutathione S-transferase (GST) enzymes are part of the cells' defence mechanism against harmful endogenous and environmental chemicals. GST disposes potential mutagens formed after UV-induced oxidative stress in the skin. Another gene involved in the detoxification of photosensitising agents is the one encoding cytochrome P450 (CYP2D6). Polymorphisms of GST as well as of CYP2D6 have been correlated with increased risk of developing $\mathrm{BCC} .{ }^{22}$ 


\section{Histogenesis}

Many theories have been proposed as to the site and cell origin of the $\mathrm{BCC}$. These have included basal cells of the epidermis, basal keratinocytes of the infundibular and outer root sheath of the hair follicle, interfollicular basal keratinocytes, dormant primordial epithelial stem cells, pluripotent epithelial cells in the basal layer that persist throughout life, cells of the pilosebaceous unit and cells of other appendageal structures. ${ }^{41-44}$ The diversity of opinions may in part be explained by the histological subtype of BCC studied. Clonality analysis concludes that BCCs are monoclonal neoplastic growths of epithelial cells, embedded in a connective tissue stroma that is at least in part of polyclonal origin. ${ }^{45}$ An observation suggesting that uncommitted stem cells are the most likely cells of origin is that sunlight exposure in childhood may contribute to tumours many decades later. The ultimate source of stem cells is the bulge region of the outer root sheath of the hair follicle. ${ }^{41}$ Histologically, BCC may show a strong resemblance with hair follicles and resembles trichoepithelioma, which is a benign hair follicle tumour. The hair follicle hypothesis is further supported by the fact that BCC seldom occurs on non-hairy skin. Supporters of this theory could debate whether $\mathrm{BCC}$ is a misnomer and whether it would be more appropriate to use the name 'hair follicle tumour' or 'hair follicle stem cell tumour'.

\section{Clinical and histopathological characteristics}

A typical $\mathrm{BCC}$ has morphological features that make the $\mathrm{BCC}$ recognisable to the trained clinician by close inspection combined with dermoscopy. $\mathrm{BCC}$ has several clinical manifestations, corresponding with different histological subtypes. The most common presentation is the pearly papule or nodule with teleangiectasia and sometimes, central ulceration, known as nodular BCC ( $\mathrm{BBCC}$ ). An erythematous, slightly scaling, well-demarcated patch with a thread-like pearly border is known as superficial BCC (SBCC). The morpheic, or sclerosing subtype has a scar-like appearance with ill-defined borders, resembling scleroderma. BCCs may occasionally be pigmented. Depending on the degree of pigmentation, BCC may resemble seborrheic keratosis or even malignant melanoma. The diagnosis and tumour type can be assessed by taking a representative punch biopsy of 3-4 $\mathrm{mm}$ for histopathological examination. $5,36,46$

The clinical differential diagnosis of $B C C$ is shown in Table 1. Illustrations of the clinical and histological features of these main subtypes are shown in Figures 1 and 2. 
Non-pigmented melanocytic nevus

Squamous cell carcinoma

Pyogenic granuloma

Malignant melanoma

Seborrheic keratosis

Merkel cell carcinoma

Actinic keratosis

Bowen's disease

Psoriasis

Seborrheic dermatitis

Table 1 Clinical differential diagnosis of BCC

Histologically, BCC typically consists of masses of neoplastic cells with well-defined peripheral contours. These masses of basaloid cells can confluent to large fields ( $\mathrm{nBCC}$ ), multiple small fields (micronodular BCC) or lie superficially with connection to the epidermis (SBCC) within a slightly myxoid stroma. Peripheral pallisading is a typical feature, as are retraction clefts that are the result of histopathological preparation. Morpheic BCCs are composed of irregular small, elongated tumour nests infiltrating in the surrounding tissue. Peripheral pallisading and retraction clefts are less common in this tumour type, and the tumour stroma shows sclerosis with thick collagen bundles (Figure 2).

Several histopathological subtypes can be differentiated. Wade and Ackerman classified 26 histological variations in BCC. ${ }^{47}$ Rippey has since proposed a simplified classification system that is easier to use in clinical practice, and is more uniform and reproducible. ${ }^{48}$ The most common type of BCC is the nodular subtype (incidence $45 \%-60 \%$ ). Pigmented BCCs often display a nodular growth. The second-most common type is the superficial subtype, with an incidence of $15 \%-35 \%$. More aggressive types are the infiltrative subtype $(10-20 \%)$, the morphea subtype (approximately $9 \%$ ), micro-nodular subtype (15\%) and adenoid type. ${ }^{48,49}$ A classification with direct clinical implications could be to classify as nodular, superficial, and infiltrative BCC. ${ }^{50}$ However, as the term infiltrative is a misnomer, it is preferred to use the term aggressive. ${ }^{51} \mathrm{BCC}$ can show more than one histological subtype, the so-called mixed type BCC. Frequencies up to $76 \%$ have been described recently in excised specimens. ${ }^{52,53}$

The aggressively growing BCCs are more frequently noted in patients under the age of 35 years than in those older than this. ${ }^{54}$ When looking at the anatomical distribution of BCCs it is found that more than two-thirds of all tumours occur on the head and neck. ${ }^{55}$ Whereas 
superficial BCCs occur mainly on the trunk and extremities in more than $60 \%$ of patients, nodular BCCs appear mostly in the head and neck region. Age and sex also appears to influence the incidence of subtypes. The frequency of superficial BCCs is higher in females and they are found more frequently in younger patients than nodular BCCs. ${ }^{56,57}$

BCC grows continuously and has a tendency to follow fascial planes, periosteum, perichondrium and eyelid tarsal plates. Nerve sheaths are also a means of spreading, as are embryonic fusion planes. ${ }^{58-61}$ Tumours arising in these fusion planes and with perineural extension demand special attention, as they may invade to unexpected depths. Examples of these sites are nasolabial folds, the columella of the nose and the pre-auricular area. All risk areas can be drawn as an ' $\mathrm{H}$ ' on the face, the so-called ' $\mathrm{H}$-zone', as illustrated in Figure 3.

The $\mathrm{H}$-zone is important for two reasons. First, it is an area with higher recurrence rates. Second, the area is of great functional and cosmetic importance. The recurrence rate depends on the size of the tumour and is often due to sub clinical spread that should be identified by a thorough histological evaluation of the peripheral and deep margins. The chances of sub-clinical spread is higher for the micro-nodular and morphea subtypes. ${ }^{62.63}$ Higher recurrence rates are found especially in morpheic $\mathrm{BCCs}{ }^{63}$ In a series of primary BCCs reported by Germann et al. $14 \%$ were the morphea subtype, in contrast to $88 \%$ of their recurrent BCCs which were the morphea subtype. ${ }^{64}$

It is important to realise that distinguishing between truly recurrent, residual and adjacent primary tumours is sometimes difficult. ${ }^{65}$ An unsuspected aggressive pathologic growth pattern may not be detected after a superficial biopsy and the BCC may recur if the initial therapy for the diagnosed non-aggressive subtype is inadequate for the aggressive part. ${ }^{52}$
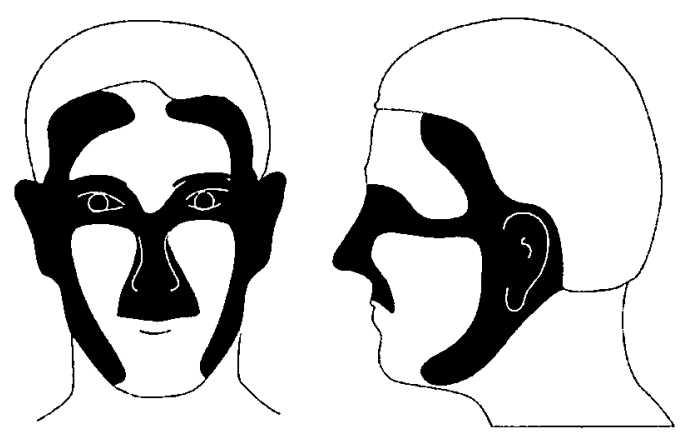

Figure $3 \mathrm{H}$-zone 

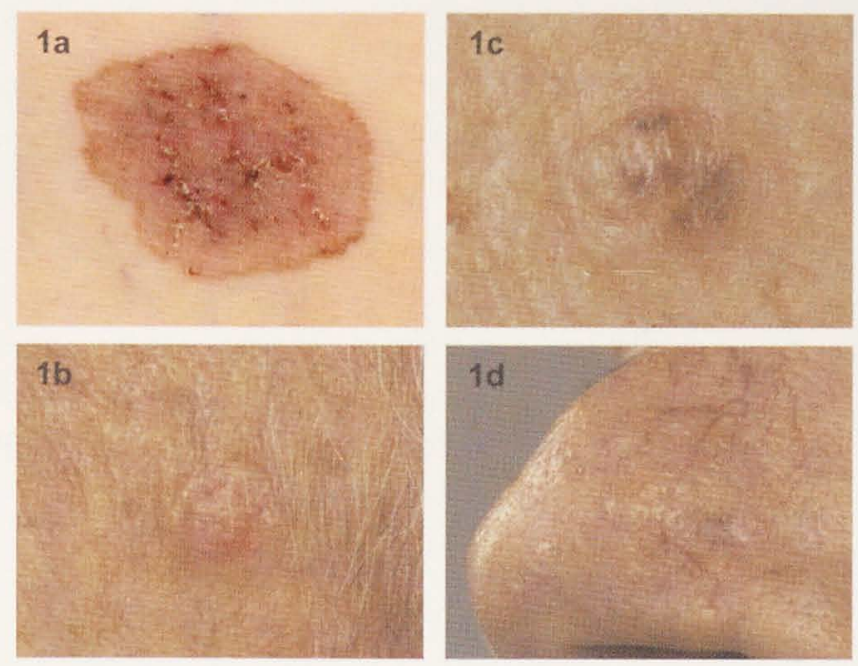

Figure 1

1a. Clinical appearance of $\mathrm{sBCC}$ on the back. $1 \mathrm{~b}$. Clinical appearance of $\mathrm{nBCC}$ on the temple. 1c. Clinical appearance of a pigmented BCC on the forehead. 1d.Clinical appearance of an ill defined aggressive (morphea-type) BCC on the nasal ala.
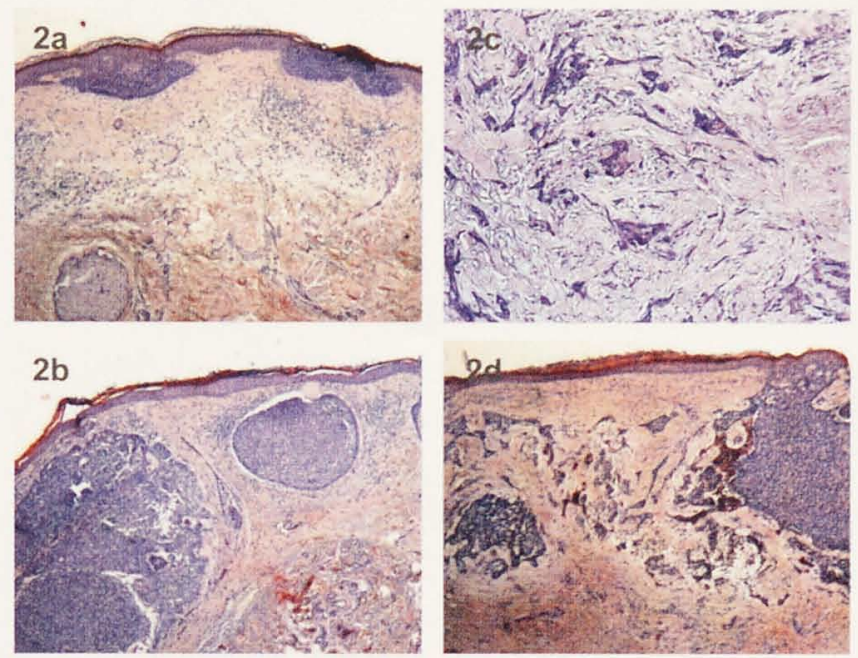

Figure 2

2a. Histological appearance of sBCC. 2b. Histological appearance of nBCC. 2c. Histological appearance of an aggressive (morphea subtype) BCC. 2d. Histological appearance of a mixed-type (nodular and infiltrative) BCC. 


\section{Metastasis}

Clinically, BCC is a slowly growing, but locally invasive and destructive tumour that, if left untreated, may lead to functional and cosmetic impairment, as around $80 \%$ of all BCCs are located in the head and neck area. Metastasis is rare (less than 1\%) and is said to correlate more to the size than to the histological subtype of the original tumour. ${ }^{66}$ Risk factors for the development of metastatic BCC include a history of a persistent tumour, refractory to conventional methods of treatment and previous radiation treatment. Metastases often involve regional lymph nodes, lungs, bone and skin. The most common theory for the low rate of metastasis is that $B C C$ is a stroma-dependent tumour; without stromal tissue there is no continuous growth of the malignant cells. ${ }^{67}$ Death from BCC occurs not only and rarely from metastasis, but also from unlimited tumour growth. ${ }^{68}$

\section{Treatment}

$\mathrm{BCC}$ has large social implications. Patients have to deal with often painful and sometimes disfiguring treatments, fear of recurrences and the advice for long-term follow-up, all of which may lead to a decrease in the quality of life.

The health care costs of the treatment, after care, absence from work and follow-up of $\mathrm{BCC}$ are very high, and it is becoming more and more important to try to keep these costs down without compromising the success of the treatment. ${ }^{50}$ in general, the number of recurrences after treatment (generally around $10 \%$ ) are high, which forces us to aim at high clearance rates, preferably 95 to $98 \%$, in the first treatment. Any subsequent managed recurrent lesion has a further enhanced risk of recurrence, due to a more aggressive growth pattern. ${ }^{69}$

There are many treatment modalities available for $\mathrm{BCC}$ at the present time, including surgical excision (SE), Mohs' micrographic surgery (MMS), cryosurgery (CS), photodynamic therapy (PDT), radiotherapy (RT), curettage-electrodessication (CE) and topical immunotherapy (Imiquimod). In addition, there are less well-established or experimental therapies such as topical 5-fluorouracil, intralesional interferon, systemic retinoids, carbon dioxide laser, pulsed dye laser, cisplatin, cyclopamine, tazarotene, $T 4$ endonuclease and

- ajoene. ${ }^{70-78}$

Considering the high incidence of this tumour as mentioned above, it is surprising how few randomised controlled trials (RCT) comparing treatment options for BCC have taken place. Fortunately, they have increased in number in recent years due to better awareness of the importance of this evidence-based medicine.

Deciding on a particular treatment should be based on tumour-, patient-, as well as doctor dependant factors. In all cases, the best available therapy with the lowest recurrence rates must be aimed for. In addition, functionality, cosmetic results, after-care and possible complications, and the patients' preferences must be evaluated for each patient individu- 
ally. Therapy for BCC is becoming increasingly customised, especially with the growing population of young patients, who are mostly well informed. Cosmetic results are of greater importance, particularly in the head and neck area, to these younger and often female patients. As the health care costs increase, the costs of a treatment should also be taken into consideration.

\section{Aims of the thesis}

One of the main motivations of the research behind this thesis was to contribute to evidence-based medicine by performing a randomised controlled trial (RCT) comparing one of the most established treatments, cryosurgery, to standard surgical excision (Chapter 2.1). Cryosurgery (CS) and surgical excision (SE) have been the most frequently applied therapies during the last decades, with the main advantage of surgery being the histological control afterwards.

Is cryosurgery for non-aggressive type BCC as effective as surgical excision?

In the USA, Mohs' micrographic surgery (MMS) is far more frequently applied than it is in Europe and it is being applied for a broader range of indications. ${ }^{79}$

Since MMS is much more expensive and time-consuming than standard excision, the long-term results of MMS were assessed in one of the largest Mohs' clinics in the Netherlands, in order to further define the indications for MMS (Chapter 2.2).

Is Mohs' micrographic surgery an effective treatment for BCC?

Coinciding with the increasing incidence of $\mathrm{BCC}$, patients tend to develop more tumours at a younger age. This younger group of patients in particular wishes to be well informed about the effectiveness, duration and after-care of all the possible treatments, the possible complications, and the cosmetic results. Electronic web pages increasingly play an important role in providing this information. The treatment of $\mathrm{BCC}$ becomes more and more customised and it is important that the dermatologist is aware of and reacts to this tendency. The application of the more recently established PDT and imiquimod as adjuvant therapy after MMS was tested in order to show that combinations of therapies are possible in certain circumstances (Chapter 2.3). 
May Mohs' micrographic surgery be combined with other, non-invasive, treatment modalities in patients with large, ill-defined BCC with combined subtype?

After performing surgery for $\mathrm{BCC}$, defects can be closed by primary suturing or by using transposition or rotation flaps or skin grafts. As well as the functional result, the cosmetic result and burden for the patient are important. The effect of systemic azithromycin on the survival of full thickness graft on the nose was examined from this viewpoint (Chapter 2.4).

Does systemic azithromycin improve the survival of full thickness grafts on the nose?

Although photodynamic therapy (PDT) is not a novel therapy, it has gained large popularity within dermatology, as well as within other disciplines during the last decade. One of the practical advantages of PDT in general lies in the time saving aspect for the dermatologist. After having built up experience with the treatment, it is possible to have a nurse (practitioner) performing the application of the precursor and performing the illumination, under supervision. The results of PDT in BCC reported in the literature vary due to differences in the execution of the treatment. Several studies show good results, especially for superficial BCC (SBCC). The preliminary results of a randomised controlled trial for the comparison of photodynamic therapy to surgical excision in nodular $\mathrm{BCC}(\mathrm{nBCC})$ which is still ongoing are presented (Chapter 3.1).

Is photodynamic therapy for nBCC equally effective as surgical excision?

PDT is painful and, in practice, this pain can be a limiting factor for the application of PDT in large tumours, certain localisations and in heavily actinic damaged skin. Attempts were made to further investigate the pain in the treatment of $\mathrm{nBCC}$ by quantifying the intensity, assessing the pain characteristics and comparing within localisations and sizes (Chapter 3.3) 
The optimum illumination time, illumination frequency, total light dose, and fluence rate are still under investigation, as well as the different precursors that are being used. The efficacy of PDT based on 5-aminolevulinic acid (ALA) and that of the commercially available methyl ester of aminolevulinic acid (MAL) was assessed in order to compare the two most frequently used substances (Chapter 3.2).

Are photodynamic therapy performed with methylaminolevulinate and PDT performed with aminolevulinate equally effective with regard to efficacy and pain?

A systematic overview of most treatments of $\mathrm{BCC}$, with their working mechanisms, indications and recurrence rates is given as introduction to this thesis (Chapter 1 ). 



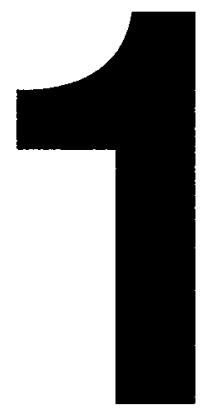

\section{Review on treatments of basal cell carcinoma}

\section{Based on the following publication}

Kuijpers DIM, Thissen MRTM, Neumann HAM. Basal cell carcinoma: treatment options and prognosis, a scientific approach to a common malignancy. Am J Clin Dermatol. 2002;3(4):247-59. 


\section{Basal cell carcinoma: treatment options and prognosis}

\section{Abstract}

The incidence of basal cell carcinoma is rapidly increasing and many treatment modalities are available, both surgical and non-surgical, both traditional and experimental. The treatment modality used, is both tumour and patient dependent. To be able to make a choice for therapy, evidence-based research is necessary. An extensive review of the literature concerning treatment modalities of $\mathrm{BCC}$ revealed that the number of these randomised, controlled long-term follow-up studies is limited.

In this chapter an overview is given of the most important treatment modalities and their effectiveness and prognosis. Based on the results of the available studies, surgical excision, Mohs' micrographic surgery and cryosurgery are the three standard therapies to choose from. Until recently, other treatment modalities were considered second choice. However, there are promising treatment options, such as photodynamic therapy and Imiquimod, of which the long-term results have to be awaited. Special attention is needed for the treatment of the more aggressive subtypes such as the micronodular, infiltrative and morpheic, the larger basal cell carcinomas and the recurrent tumours. 


\section{Introduction}

There are many different and well-accepted treatment modalities used in the management of basal cell carcinomas (BCC). In making a choice for therapy, different factors should be taken into account, both tumour dependent factors such as localisation, size and histological type of tumour, history of previous treatments, as well as patient dependent factors such as age, skin colour and general health of the patient. Besides this, the experience of the dermatologist is of importance. ${ }^{80}$ In the second place, the cosmetic results, aftercare and possible complications, costs, and the recurrence rates for each therapy must be evaluated for each patient individually. Presently, there are many treatment modalities available for treating BCC. The main goal for therapy is complete eradication of the tumour. Recurrences have to be avoided, because they are difficult to treat. A distinction can be made between therapies with post-treatment histological control; these are the surgical treatments, and those without histological control, the non-surgical treatments. We performed an extensive review of literature, with the aid of Pubmed and Medline databases, at which the language of the articles was limited to English, German and Dutch. The most important and widely accepted treatment modalities will be discussed further in this article, together with the results of important studies. First, some general remarks about resection margins and the histo-pathological examination will be made.

\section{Histology and resection margins}

The principal of taking a resection margin of normal appearing skin in the treatment of $B C C$ is based on the assumption that the tumour extends beyond its clinically apparent borders. The degree of clinical invisible extension varies with the histopathological subtypes and is an important factor for final treatment. ${ }^{81}$ The wider the margin, the greater the possibility that the tumour is removed entirely. Resection margins must be taken into account regardless of the treatment modality, micrographic surgery excluded. Breuninger calculated the safety margins in relation to the confidence of entirely removing the tumour in $95 \%$ of the cases (Table 1.1). ${ }^{82}$ Wolf and Zitelli assessed that for tumours less than 2 $\mathrm{cm}$ in diameter, a minimal resection margin of $4 \mathrm{~mm}$ is necessary to eradicate the tumour in more than $95 \%$ of cases. Margins of 3 and $2 \mathrm{~mm}$, result in a complete eradication of respectively $85 \%$ and $75 \% .{ }^{83}$ No differentiation was made between various subtypes, so that for high risk BCCs larger margins would be necessary, even up to $13 \mathrm{~mm}$ for a $20 \mathrm{~mm}$ lesion diameter. ${ }^{84}$ When this is performed consequently, unacceptable large defects occur, especially in the face. For this reason $3 \mathrm{~mm}$ resection margins for small $(<10 \mathrm{~mm})$ and 5 $\mathrm{mm}$ resection margins for larger $(10-20 \mathrm{~mm})$ nodular and superficial BCC in the face and $5 \mathrm{~mm}$ resection margins for nodular and superficial tumours elsewhere on the body are recommended. ${ }^{85}$ The margins should be measured accurately and drawn onto the skin before treatment. 


\begin{tabular}{|l|l|}
\hline Tumour diameter $(\mathrm{mm})$ & Resection margin $(\mathrm{mm})$ \\
2 & 2 \\
4 & 3 \\
6 & 4 \\
8 & 5 \\
10 & 6 \\
12 & 8 \\
14 & 9 \\
16 & 10 \\
18 & 11 \\
20 & 13 \\
\hline
\end{tabular}

Table 1.1 Relationship between clinical tumour diameter and resection margin for SE of $\mathrm{BCC}^{85}$

The surgically excised tumour as well as the biopsy specimen should be fixed in formalin and submitted for histopathological examination. After traditional staining with haematoxylin-eosin, the tumour cells become clearly visible. In difficult cases, were it is doubtful whether the tumour is from epithelial origin or not, immunohistochemical staining with cytokeratin antibodies may give additional information. The possibility of different cytokeratins as histopathological marker (such as cytokeratin 8 ) is being investigated, but the expression in the tumour seems to be highly dependent on the monoclonal antibodies used to detect the cytokeratins. ${ }^{86} \mathrm{Up}$ to now, there is no specific tumour marker for BCC. Staining with $\mathrm{Bcl}-2$ antibodies can differentiate between basal cell carcinoma, squamous cell carcinoma and tricho-epithelioma. ${ }^{87}$

Conventional histopathological examination has an important disadvantage, which is necessary to realise, when interpreting results in cure rates.

Because the pathologist can impossibly examine the entire specimen microscopically, the excised tissue is divided into a number of blocks. Subsequently, these blocks are cut into several vertical sections across their narrowest axis. This technique is called the 'bread loaf' method (Figure 1.1). Abide et al. calculated that with this standard histological examination only $0,01 \%$ of the resection surfaces will be visualised.$^{88}$ It is obvious that the positive predictive value of 'margins contain tumour cells' is nearly $100 \%$. But no conclusions about completeness can be drawn in cases where no tumour cells are found on the section margins. Especially in high-risk tumours different histo-pathological techniques are preferred like the 'flounder-technique' and the Mohs' micrographic surgery, which make in theory, an $100 \%$ projection of all lateral borders and the bottom possible. These techniques will both be discussed further in this article. 


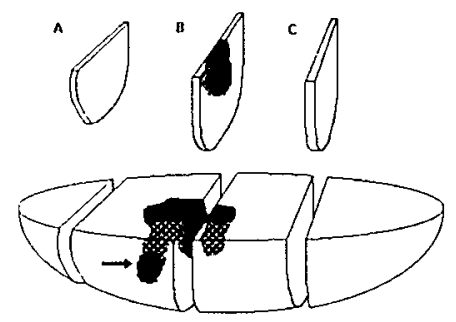

Figure 1.1 Schematic reproduction of the bread-loaf technique, demonstrating the limitation of the technique. Routine histopathological examination of the resection margins after surgical excision of BCC usually will be performed using the bread-loaf technique. At standardised distances, the excision specimen is cut into several vertical sections. In this way, tumour rests in the resection margins can be missed. In the sections $A, B$ and $C$, the cutting edges in the front are free of tumour celis, but in the non-examined parts $(\rightarrow)$ tumour cells are still present

\section{Therapy and techniques Surgical excision}

The scalpel has already been used for centuries to cure diseases. Over the last decades, surgical techniques have been refined and medical practitioners became more experienced. Nowadays, surgical excision (SE) is worldwide the most frequently used technique of treating BCCs. Surgical excision makes it possible to assess the completeness by histopathological examination and is therefore often first choice therapy for BCCs. In dermatology, surgical treatment can generally be performed under local anaesthesia. The tumour is excised down into the subcutaneous fat, or the next involved anatomical layer.

The excised tumour should be marked with a suture for subsequent orientation, or even better, the bottom and cutting edges can be marked with different colours of tissue dyes. ${ }^{84}$ Closure of the defect is performed either primarily, by placing a graft or by secondary healing. Primary closure consists of direct suturing of the different layers of the wound, or when this is not possible because the created defect is too large, and therefore holds too much tension on the wound edges, by creating a flap. The flaps can be divided in advancement, rotation or transposition-flaps. In case of difficult primary closure, a split- or full-thickness graft can be used.

Before closure of the defect, especially in cases where reconstruction is performed with a flap, information about completeness is mandatory. By movement and transposition of the anatomical layers, the localisation of the original defect will be hindered and an eventually following radical re-excision would be very difficult to perform. When there is doubt about the completeness, secondary healing or closure after definitive histopathological result needs to be considered.

The management of incompletely excised $\mathrm{BCC}$ has been discussed in literature. There is evidence suggesting that total removal of the tumour is not necessary to effect cure and 
that up to two-thirds of the incompletely excised BCCs that are not re-treated do not recur. $^{89.90}$ However, re-excision specimens may show no residual tumour, but with the knowledge that only less than $1 \%$ of the tissue is examined, this gives no guarantee for complete treatment. And, in case of residual tumour cells at the deep margin, a false negative result of the re-excision can occur, when this is performed too superficially.

Several other studies have strongly recommended the immediate re-treatment of the tumour, especially when the surgical defect has been repaired using skin flaps or grafts. ${ }^{91}$ Re-excision with MMS is probably the treatment of choice when the BCC has tumour cells on the deep margin, and is of an aggressive type. ${ }^{36,92.93}$

There are numerous reports to be found in literature concerning the effectiveness of various treatment modalities, including SE. There are only few studies with a standard follow-up period of five years, and even less prospective, randomised studies. ${ }^{94.95}$ Studies reporting recurrence rates based on follow-up shorter than five years give an understatement of the true recurrence rate. Rowe et al. ${ }^{96}$ calculated that the average five years recurrence rate is more than two times that of short-term follow-up studies (two years). Independent of the modality of treatment, recurrences after treatment of primary $\mathrm{BCC}$, one third of the recurrences occur in the first year, $50 \%$ within the first two years and only two-third within three years follow-up. Eighteen percent recurrences occurs between the fifth and the tenth year of the follow-up. Other retrospective analysis resulted in comparable figures. ${ }^{97}$ Cumulative five years recurrences rates for SE of primary BCCs vary from $3.2 \%$ to $8.0 \% .64 .79,94,98,99$ For recurrent $\mathrm{BCCs}$, the re-recurrence rate is found to be $6.7-11.6 \%{ }^{79.94}$ Randomised controlled trials comparing SE to another treatment modality result in recurrence rates ranging from $2.9 \%$ after 30 months follow-up to $1 / 174$ after four years. ${ }^{100.101}$

In general, cosmetic outcome is excellent following SE. ${ }^{94}$ In comparison to cryosurgery, SE as a treatment for primary uncomplicated BCCs, results in a significantly better cosmetic outcome. ${ }^{102}$

\section{Micrographic surgery}

Micrographic surgery refers to the serial excision of skin cancer and precise mapping of the operation field followed by histopathological examination. It is a precise method of removing only malignant tissue by examining all cutting edges. The following important micrographic techniques have been developed during the years and will be discussed below: Mohs' micrographic surgery (MMS), the flounder technique and the Munich technique. 


\section{Mohs' micrographic surgery}

In the 1930s, Dr. Frederic Mohs developed a technique for chemical in vivo fixation of cutaneous tissue, followed by serial excision and microscopic control. He fixated the tumour in vivo with zinc chloride paste, permitting it to be excised carefully. The disadvantage of this 'chemosurgery' was that only one single application followed by one single Mohs' cut could be performed, so it could have taken days to completion. Also the fixation in vivo was very painful for the patient. This technique of fixed-tissue MMS has gradually been replaced by that of fresh-tissue MMS. ${ }^{58,103}$ In this technique with fresh-frozen sections, the chemical fixative was no longer used and patients could be treated in one session. Today, more than $95 \%$ of MMS is performed with the fresh-tissue technique. However, for certain tumours, the fixed tissue technique remains valuable, for example BCC invading bone, meninges or brain. ${ }^{5 B}$

The procedure begins with a precise drawing of the tumour on a map to ensure correct positioning during the operation, followed by careful assessment and marking of the clinical borders of the tumour. After these preparations, the tumour is often debulked by curette or scalpel. This is not counted as the first layer of tumour removal. Then the curetted wound, including a $2 \mathrm{~mm}$ margin of epidermal layer is excised at an angle of approximately $45^{\circ}$. The excised tissue is cut into small parts and the cutting edges are coloured to allow correct orientation of the removed tissue. Subsequently the excision specimens are frozen in the cryostat and at the same time the cut surfaces are carefully flattened by pressure. In this way it is possible to cut the whole resection margin (both deeper and epidermal layer) into horizontal sections. Now $100 \%$ of the section edges can be evaluated, in contrast to only a small part which is visualised with the conventional histological examination. ${ }^{88} \mathrm{Ac}-$ curate mapping of the numbered specimens is performed on the anatomical picture.

Following the histo-pathological examination of the first cut, sequential cutting rounds will be performed until all resection margins are free of tumour cells. After the tumour has been totally removed, the final defect will be closed either primarily or by secondary healing, as described before.

As MMS offers a high cure rate and maximal preservation of normal tissue, it therefore is a valuable treatment modality for BCCs in the $\mathrm{H}$-zone. ${ }^{58}$ Also for recurrent $\mathrm{BCC}$, MMS has been advised as first line treatment. ${ }^{104,105}$

The indications for MMS in the treatment of BBCs are listed in Table 1.2. 


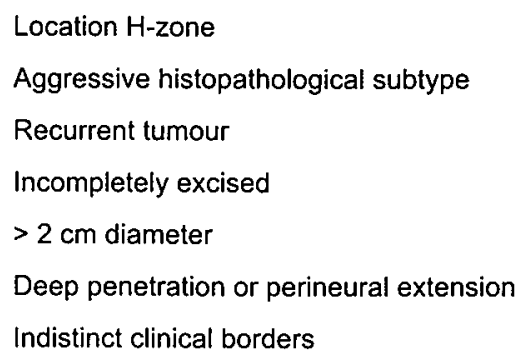

Table 1.2 Indications for MMS as first-line treatment for BCC.

Recurrence rates range from 1 to $6.5 \%$ for primary $B C C$, and 2 to $10 \%$ for recurrent $B C C$ in several retrospective studies over a 3 to 5 year time frame. ${ }^{58,65,79,105,106}$ One study reported a five-year cure rate of $97.1 \%$ in a series of 929 BCCs of the external ear. ${ }^{107}$ Furthermore, one large series of $1414 \mathrm{BCCs}$ on the eyelid showed a five-year cure rate of $99.4 \%$ for primary tumours and respectively $92.4 \%$ for recurrent eyelid tumours. ${ }^{108}$

There are only a limited number of prospective, randomised studies evaluating MMS. Julian and Bowers found within a five years follow-up period an overall recurrence rate of $3.8 \%$ in 200 high risk basal cell carcinomas, $1.7 \%$ for primary tumours and $4.8 \%$ for previously treated tumors. ${ }^{104}$ Smeets et al. calculated a recurrence rate of $1.9 \%$ after MMS for primary BCC in comparison to $2.9 \%$ after surgical excision after a follow-up of 30 months in a large series. ${ }^{100}$

Besides all the advantages of Mohs' surgery, there are a number of relative disadvantages. MMS is time consuming and labour intensive. It might be more expensive than non-surgical modalities like cryotherapy and curettage-electrodessication, because of the need for special laboratory processing and microscopic examination. There are only a limited number of qualified Mohs' surgeons. A cost analysis by Cook and Zitelli however showed that MMS is as cost effective as traditional surgical excision, taken into account the 10-fold higher number of recurrences with SE that also need to be re-treated. ${ }^{109}$ Other studies found significant higher costs for MMS and concluded that at present, it does not seem cost-effective to introduce MMS on a large scale for BCC. ${ }^{110}$

\section{Flounder technique}

Also the flounder technique, as described by Breuninger, offers a three dimensional histological projection. ${ }^{82}$ With this method, the margins of the tumour are folded down on a single plane after the central tumour body has been excised, before the excised material is processed for routine histopathological examination. The entire vertical margin of the tumour section, including epidermis, and the bottom are displayed histological in a single, continuous section. The flounder technique differs from the Mohs' surgery in the fact that 
the tumour, including a preoperative calculated margin (depending on the tumour diameter) of healthy tissue is removed, instead of saving as much healthy tissue as possible. Then again, the flounder technique may not be as time-consuming as MMS. Despite the good results reported by Breuninger, no other groups have confirmed the value of this technique.

\section{Munich technique}

In Munich, Germany, a micrographic technique has been developed, at which the whole excised tumour is being sectioned, from the deep margin until the epidermis, parallel to the skin surface. ${ }^{11}$ The sections are being cut with intervals of 150-200 $\mu \mathrm{m}$, and therefore might be less sensitive than the other micrographic techniques.

\section{Cryosurgery}

Cryosurgical techniques have already been used since the second half of the nineteenth century. Several cryogenics have been developed like cold saline solution, liquid oxygen, nitrogen, liquid air and carbon dioxide. Liquid nitrogen was found to be the most potent cryogen and was initially applied by the swab method. ${ }^{112}$ In the 1960's, the first closed system apparatus, using cryoprobes with liquid nitrogen, was described by neurosurgeon Cooper. Further development resulted in the open-cone-spray method with neoprene cones as it is still used today. ${ }^{113}$ Thermocouples can be used to control the temperature deep in the tissue, which requires a lot of experience. When no thermocouple is used, the procedure should be standardised. This can be done by standardising the freezing and thawing times of the cryo-cycle.

The basic principal of cryosurgery (CS) is based on the induction of selective necrosis by using cryogenic materials. Each freeze and thaw cycle leads to change in tissue texture and even to destruction. Rapid freezing and long thawing periods cause the most severe injury. Zacarian found $-50^{\circ} \mathrm{C}$ to be the optimal temperature to obtain effective cryonecrosis of malignant tumours. Sensitivity for freezing is different for the different cell types of the skin. Keratinocytes are destroyed at lower temperatures $\left(-20^{\circ} \mathrm{C}\right)$ compared to melanocytes $\left(-4\right.$ to $\left.-7^{\circ} \mathrm{C}\right)$. Cartilage is relative unsusceptible for freezing, which makes CS very suitable for treating smaller BCCs on the eyelids and parts of the ear. ${ }^{14}$ Fortunately, also vital structures as tear ducts and nerves are preserved.

CS is performed under local infiltration anaesthesia with lidocaine $1 \%$ with epinephrine. The added epinephrine leads to vasoconstriction and results in more effective long thawperiod. Prior to the freezing cycles, the tumour is curetted carefully to diminish to tumour mass. When using the open-cone-spray method, the cone is placed over the curetted tumour with a safety margin of $5 \mathrm{~mm}$. Best results are being achieved by a double freezing-thawing cycle, with the thawing time being at least three times the freezing time. 
A single cycle gives better cosmetic results, but more recurrences. Three cycles will enlarge the effectiveness, but will leave cosmetically unacceptable scars. ${ }^{115}$ After treatment, the wound heals in one to several weeks with considerable oedema, during which time dressings need to be changed several times a day. When healed, a persistent hypo-pigmented scar resides. This makes CS cosmetically less attractive to darker skin types.

In literature four prospective studies with a long follow-up (five years) reporting results of CS could be found, three regarding BCCs on the eyelids and one regarding on the nose. The latter demonstrated two recurrences in 50 treated non-morphoeiform basal cell carcinomas. ${ }^{116}$ The studies reporting BCCs on the eyelids varied in cumulative five years recurrence rates from 0 to $16.5 \%$ in BCCs larger than $1 \mathrm{~cm} .{ }^{114,117,118}$

Recurrence rates in randomised controlled trials vary from $4.2 \%$ to $39 \%$ after a two year follow-up. ${ }^{102.119-121}$ Retrospectively, one series of 269 BCCs treated with CS, which were followed for five years, showed a recurrence rate of $7.5 \%$, whereas a short follow-up resulted in a much lower recurrence of $3.7 \% .{ }^{96}$ One other retrospective study reported 5 recurrences in $522 \mathrm{BCCs}$ after five years, without mentioning loss to follow-up. ${ }^{122}$

$\mathrm{CS}$ is time saving, relatively inexpensive and easy to perform in experienced hands. $A$ disadvantage is the lack of histological control for the completeness of the treatment.

Relative contraindications for CS are locations of BCC in the $\mathrm{H}$-zone, hairy scalp and eyebrows for the risk on scarring alopecia, and lower legs and feet for the risk on ulcers. Also tumour related facts as size, aggressive type, recurrence, fixation to bone and deep invasion are relative contraindications. ${ }^{23}$

\section{Curettage-electrodessication}

Another treatment modality used for basal cell carcinomas is curettage, which traditionally has been combined with cautery or electrodessication of the wound bed. The use of warmth already dates back to ancient times, when a heated rock or metal was held on to a wound to stop bleeding. Today, there is a considerable variation in performance of the curettage-electrodessication (CE) technique as there is no standardised method followed by all practitioners. The general principles are well established and consist of curettage of the tumour using curettes in successively smaller sizes. The vulnerable soft tumour tissue is easily being separated from the underlying normal dermis. Subsequently, the wound bed is dessicated (coagulated), with the electrode making direct contact to the tissue.

The heat developed by electrodessication results in the clotting of proteins and destruction of the cells. This cycle is generally repeated two or three times. ${ }^{46}$

The wound produced heals by secondary intention and tends to result in a hypertrophic scar or even keloid. ${ }^{124}$

The cumulative five years recurrence rates in primary BCCs, vary from $3.3 \%$ and $3.7 \%$ for BCCs in low-risk sites to $18.8 \% .^{124.125}$ This high rate was achieved by treating BCCs in 
early days (from 1958 to 1962) when also the large, high risk BCCs were treated by this technique. Increasing diameter of the tumour and high-risk sites were again found to be independent risk factors. The cosmetic outcome is fair to good, and tends to improve with time. As with other non-surgical treatments of BCC, there is a lack of histological control. $C E$ is cheap and fast to perform, but with an uncertain outcome.

\section{Radiotherapy}

Radiotherapy (RT) is one of the longer existing treatment modalities for $B C C$, and its use has declined over the years. As an example, in the UK $8 \%$ of the BCCs are treated with RT. In the Netherlands, dermatologists indicated in $2 \%$ of cases RT as first line treatment. ${ }^{5}$ Many radiation sources are available for radiation treatment of skin cancer. Orthovoltage (100-250 kV) and electron beams (3-16 MeV) are most often applied, while photon beams (4-10 MV) are less often used. In the past contact-radiotherapy was mainly used, but this has been replaced by the ortho-voltage and electron RT, with a decrease of side-effects as a result. the technique chosen is mainly dependent on the size, depth as well as on the anatomical location of the tumour. the energy chosen will determine the penetration depth. However, the efficacy of radiation treatment is dependent on both the total radiation dose delevered, as well as the margins applied. Whereas the fraction dose will mainly influence the cosmetic outcome of treatment as well as the late radiogenic effects. ${ }^{126}$

A recent retrospective study describes $104 \mathrm{BCCs}$ of the head radiated with low energy photons (50-100 kV) with a total tumour dose of approximately 57 Gy prescribed in $3 \mathrm{~Gy}$ fractions, in a schedule of 5 fractions per week. After a median follow-up of 37 months, no recurrences were reported, with good to excellent cosmesis and low acute toxicity at the end of the radiotherapy in $87 \%$ of tumours. ${ }^{127}$

Silverman et al. reviewed the treatment of 862 primary BCCs with RT ( 5 fractions of 680 cGy, margins at least $5 \mathrm{~mm}$ ) and found a five-year recurrence rate of $7.4 \% .9 .5 \%$ of 211 recurrent BCCs re-recurred within five years. ${ }^{128}$ Increasing diameter of the tumour was the only independent risk factor for high recurrence rates. Another retrospective study on 175 $\mathrm{BCCs}$ (both aggressive and non-aggressive) resulted in an estimated five-year recurrence rate of $15.8 \% .^{129}$

Two randomised controlled trials have been performed on RT. In one, RT was compared to $\mathrm{CS}$ in 93 patients with BCC. ${ }^{121}$ Two years after treatment, $4 \%$ of tumours treated with RT and $39 \%$ of those treated with cryotherapy had recurred. In the second randomised controlled trial, radiotherapy was compared to surgical excision in 347 patients. RT resulted in $11 / 173(6.4 \%)$ actuarial recurrences after three-five years, with good cosmetic results. ${ }^{101}$ Bad or unacceptable cosmetic results vary in literature from 10 to $30 \%$. $^{130.132}$ As the late effects of RT on the skin such as hyper- or hypopigmentation, atrophy and teleangiectasia develop with time, the cosmetic outcome declines over the years after treatment. As men- 
tioned above, the cosmetic result from RT is mainly dependant on dose, fractionation size and of course the quality of radiation. ${ }^{133}$ Lower fraction doses in combination with more exposures to achieve an equal total dose improve the cosmetic outcome and seem not to affect the recurrence rate.

Recurrent BCC after RT is known to be difficult to treat by surgical excision due to the difficult clinical delineation of the tumour in the previously treated skin. Even change to more aggressive and infiltrative tumour growth pattern has been noted after RT. ${ }^{134}$ As in other non-surgical treatments, the lack of histo-pathological control for completeness of the radio-therapeutic treatment can be regarded as a disadvantage.

Another disadvantage of RT is that it gives rise to an increased risk for secondary malignancies with an interval of 15-20 years. RT induced malignancies include skin malignancies such as squamous cell carcinoma and BCC. ${ }^{135,136}$

Other disadvantages of RT include the risk for radio-dermatitis, inconvenience for the patient for making several visits to the hospital and high costs, which even exceed those of Mohs' surgery. ${ }^{46}$

\section{Photodynamic therapy}

Photodynamic therapy (PDT) is a minimally invasive two-stage procedure that requires administration of a photosensitising agent followed by illumination of the tumour with visible light. PDT is increasingly being applied in the treatment of skin cancers and other malignancies. ${ }^{137}$ The anti-tumour effects of PDT are complex and result from the combination of direct tumour cell photodamage leading to apoptosis, necrosis and destruction of tumour vasculature and activation of an immune response. ${ }^{138.139}$ These effects are dependent on the (intracellular localisation of the) photosensitiser, the PDT dose and the cell genotype. ${ }^{139.140}$ After light activation of a photosensitiser localised in tumour tissue, reactive oxygen species, in particular singlet oxygen, are generated, which modify cellular functions or induce cell death by necrosis or apoptosis, occur. ${ }^{141}$ Figure 1.2 shows a simplified scheme of the photodynamic principle in which photo-energy is converted into chemically active species, mediated by a photosensitiser.

PDT induces apoptosis via two major pathways: mitochondria-mediated and death-receptor mediated. ${ }^{140}$

The first pathway is triggered by disruption of mitochondrial transmembrane potential and release of cytochrome-c to the cytoplasm. This allows formation of complexes called apoptosomes and activates caspases, which are hydrolytic enzymes. This leads to cell death. ${ }^{140}$ This pathway is strongly influenced by the pro- and anti-apoptotic $\mathrm{Bcl}-2$ family of proteins, although the precise interaction is not known yet. ${ }^{141}$

The second pathway is triggered when photosensitisers target the cell membrane. Cell membrane receptors are polymerised by a complex of Fas, FADD and procaspase-8, 
again eventually leading to cell death.

PDT also causes or enhances an immune response. As PDT locally damages the tumour structure, it enables influx of non-affected immune cells into the treated site, resulting in the development of a systemic anti-tumour response. ${ }^{139}$ A photosensitiser can be administered systemically, and for the treatment of skin cancer topical use is possible, with fewer side-effects. ${ }^{142}$ Also local, intracutaneous administration has been investigated. ${ }^{143,144}$

In dermatology, 5-aminolevulenic acid (ALA) as well as methyl aminolaevulinic acid (MAL) in a cream or an emulsion are used in the treatment of cancerous and pre-cancerous lesions, like BCC, solar keratosis and Bowen's disease. ALA and MAL are not the actual photosensitisers, but exogenously administered precursors that are being converted into the photosensitiser protoporphyrin IX (PpIX), which is a product of the haeme biosynthesis pathway (Figure 1.3). This process takes place in the mitochondria of the cell.

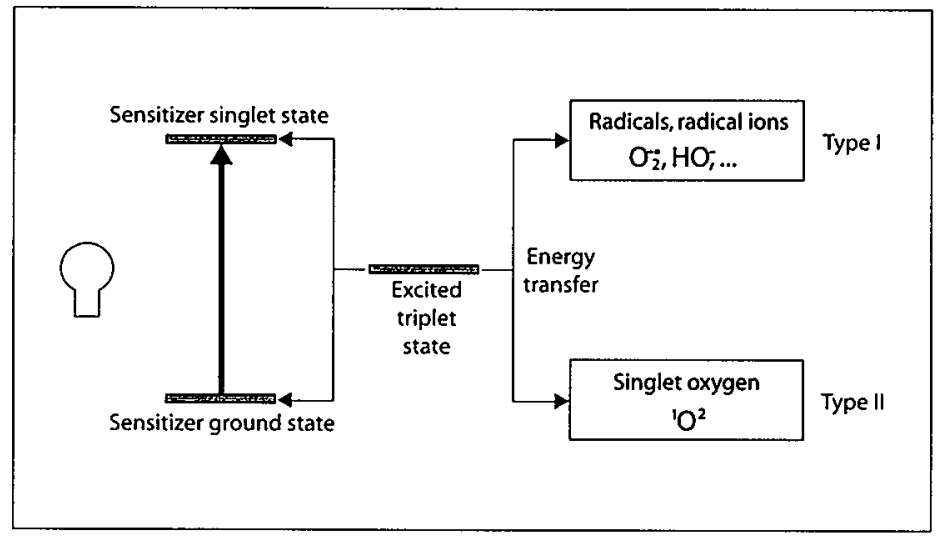

Figure 1.2 Photodynamic principle (from Rigel et al: Cancer of the Skin (c) 2005 Elsevier Inc.) A photosensitiser molecule in the singlet ground state is excited to a higher energy state, e.g. to the singlet excited state by the absorption of a photon. Molecules at the short-lived singlet state either decay back to the ground state through fluorescence emission, or undergo intersystem crossing to the excited triplet state. Molecules at the excited triplet state can undergo 2 types of reaction with surrounding molecules; an electron transfer process (type I photochemical reaction) and an energy transfer process with an oxygen molecule (type II photochemical reaction), both proceeding simultaneously and in competition. The products of the type 1 reactions are free radicals, which can react with oxygen to give various oxidised products that initiate free radical chain reactions. In the type II reaction, molecules at the excited triplet state react with ground-state oxygen to produce excited singlet oxygen $\left({ }^{1} \mathrm{O}_{2}\right.$ ) and return the photosensitiser to the ground state. The ${ }^{1} \mathrm{O}_{2}$ species is highly active in biological systems, interacting with a number of proteins, lipids and other biological molecules. The ${ }^{1} \mathrm{O}_{2}$ species can also effect cell death, which is the most important factor in the PDT-induced tissue damage. 


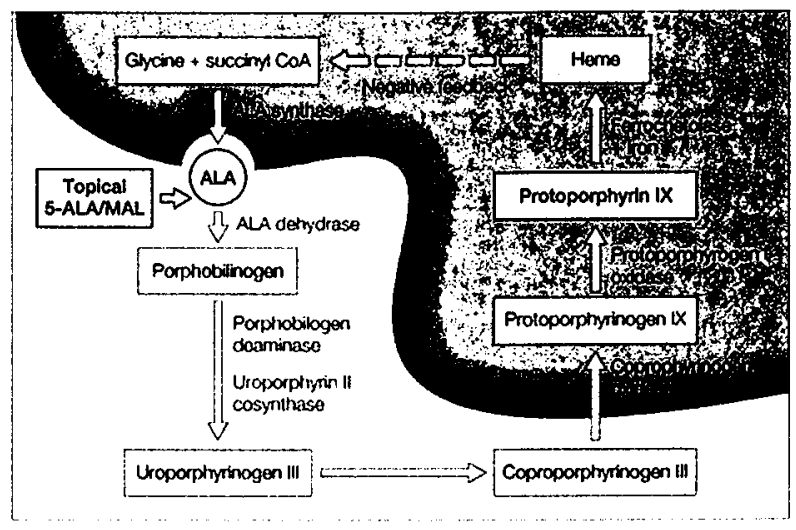

Figure 1.3 Simplified haeme synthesis pathway (from Rigel et al: Cancer of the Skin (c) 2005 Elsevier Inc.) The steps between conversion of 5-ALA via porphobilinogen and uroporphyrinogen II into copro-porphyrinogen III, the second last precursor of protoporphyrin IX, take place in the cytoplasm of the cell. The final two conversions of protoporphyrinogen III into protoporphyrinogen $I X$ and successively into $\mathrm{PplX}$ take place in the cellular mitochondrial matrix.

After application it takes 4-8 hours to synthesize an amount of PplX that is sufficient for a response to PDT. MAL has shown a higher selectivity for tumour cells compared with ALA, which is explained by a difference in cellular uptake due to an increased lipophilic structure in esterified ALA. ${ }^{145}$

In vitro studies suggest that PDT with ALA-esters is more effective than with ALA. ${ }^{146}$ However, in vivo studies show no higher $\mathrm{Pp} / \mathrm{X}$ levels induced after the esters, some authors have even found higher porfyrin levels after ALA application in AK and normal skin. ${ }^{147}$

The porphyrins exhibit a typical absorption spectrum with a high peak at approximately $405 \mathrm{~nm}$, the so-called Soret band. Visible blue light matching this band can be used for the treatment of AK. Several so-called Q-bands also exist, the last having an absorption peak at $635 \mathrm{~nm}$, matching red light (Figure 1.4). Because this red light penetrates deeper into the skin compared to blue or green light, it is recommended for the treatment of BCC. The optimum illumination time, total light dose, fluence rate and illumination frequency are still under investigation. Generally, single irradiations with total light doses between 50 and $150 \mathrm{~J} / \mathrm{cm}^{2}$ and fluence rates between 50 and $100 \mathrm{~mW} / \mathrm{cm}^{2}$ are used.

The thickness of the tumour appears to be the most important determinant of tumour response as superficial $B C C$ appears to respond better than the nodular BCCs. ${ }^{148}$ The response to the treatment probably is more restricted by the limited penetration of the sensitiser, than by the penetration of light. The penetration depth of the porphyrins-fluorescence pattern induced by topical and intra-lesional ALA (unpublished data) is inhomogenous. ${ }^{149}$ In normal skin is found that higher light dose, lower irradiance and fractionated illumination 
can increase the depth of the tissue damage. ${ }^{150}$

To improve the response to PDT of the thicker, nodular BCC, a preceding superficial debulking of the tumour mass can be performed, intracutaneous administration, fractionation of the light dose can be applied or penetration enhancers like dimethylsulfoxide (DMSO), or Ethyldiaminetetraacetate (EDTA) can be used. ${ }^{151-154}$

Illumination of sensitised skin causes a burning sensation, even pain, especially on the face, of which the mechanisms are not fully understood.

After treatment, local erythema and oedema in the treated area are usually seen. Crust formation can occur. In the following days to weeks the skin will heal with an excellent cosmetic result, without atrophy. Seldom, hypo- or hyperpigmentation, which most often is temporary, is noticed. ${ }^{150.152}$

Clinical complete response rates for superficial BCC to topical ALA-PDT of 90 to $100 \%$ are reported. ${ }^{142.150 .155 .156}$ Nodular $B C C s$ result in complete response rates of 10 to $71 \%{ }^{148.150}$ One study with a average of 17 months follow-up showed a response of $95 \% .{ }^{153}$

$B C C$ s larger than $4 \mathrm{~cm}$ in diameter have shown a poor response to ALA-PDT, perhaps due to inhomogeneous and insufficient penetration of ALA or the irradiation ${ }^{142}$ or due to deeper growth. There are only few randomised controlled trials (RCTs) on PDT and in general, a standardised PDT treatment regimen lacks. The large differences in illumination intensity and frequency, illumination devices and the used photosensitisers make the response rates and long-term results of PDT difficult to compare. ${ }^{152}$

Two RCTs regarding PDT resulted in a (histopathologically confirmed) recurrence rate of $25 \%$ and a disease-free rate of $83 \%$, both after one year of follow-up. ${ }^{119,157}$

In addition to PDT, ALA as a photosensitiser can be used for other purposes. The absorption of light energy by a photosensitiser increases the energy of its electron rendering the photosensitiser excited. The excited sensitiser can relax back to its ground state by emitting fluorescence. As the fluorescence of ALA induced porphyrins is highly selective, it offers a useful technique in detecting and delineating skin tumours with clinically undefined borders or even new tumours. This is known as photodynamic diagnosis (PDD). ${ }^{158.159}$ For detecting and delineating epithelial tumours, PDD is routinely used in only few clinics, and is recommended by some as a preoperative procedure and as a tool in follow-up, as PDD is highly sensitive in detecting tumour cells before they are clinically apparent. 


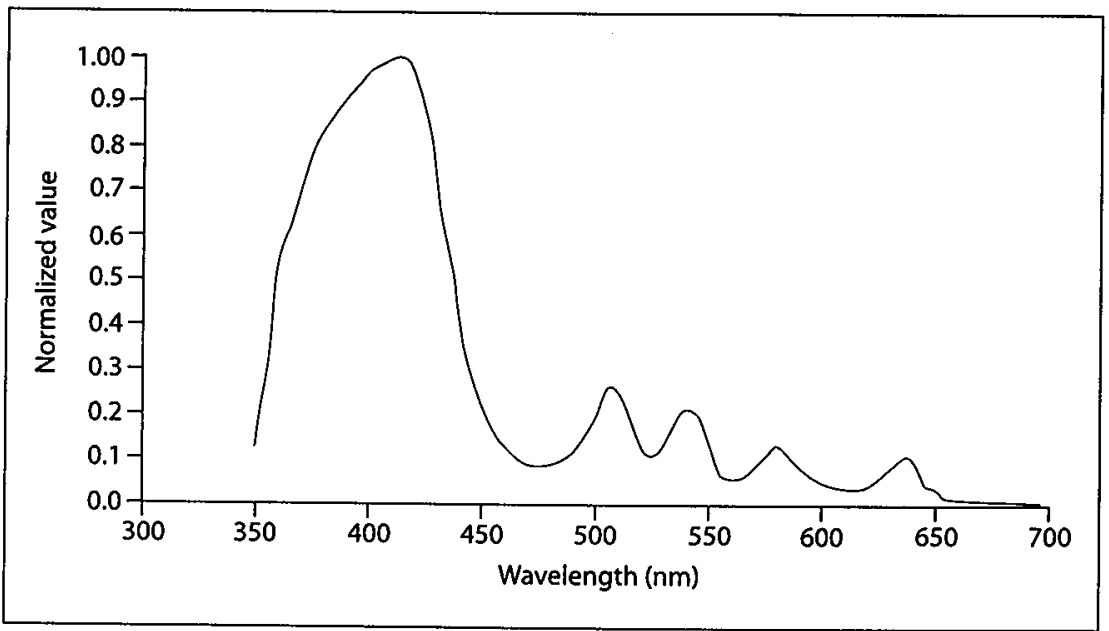

Figure 1.4 Absorption spectrum of PpIX (from Rigel et al: Cancer of the Skin (c) 2005 Elsevier Inc.)

\section{Imiquimod}

A relatively new treatment option for $B C C$ is imiquimod (imidazoquinoline $5 \%$, Aldara $B$ ). The most common indications for topical use of imiquimod are the treatment of external anogenital warts, actinic keratosis (AK) and superficial BCC (SBCC). Recently imiquimod $5 \%$ cream has been approved in the Netherlands for SBCC.

Imiquimod is a topical immune response modifier which has both anti-tumour and antiviral properties due to enhancing the innate and acquired immune system. ${ }^{160}$ As an agonist of tolllike receptor (TLR) 7 imiquimod binds to receptors, such as these TLRs, causing monocyte and macrophage stimulation which produce several cytokines, including interferon (IFN) -a, interleukin (IL) 1,6,8,10,12 and tumour necrosis factor-a. Furthermore, stimulation of natural killer cells, proliferation of $B$ cells and activation and migration of Langerhans cells occur. An indirect effect of imiquimod is the stimulation of T-helper cells to produce cytokines including IFN- $\gamma$ which can stimulate cytotoxic T-cells. These T-cells can kill virus infected cells and tumour cells and are important for establishing the immunological memory. ${ }^{161,162}$

During treatment with imiquimod there is an increase in the apoptosis index. This is based on a decline of anti-apoptotic factors like $\mathrm{Bcl}-2$, as well as an increase in pro-apoptotic stimuli (cytotoxic $T$ lymphocytes, natural cytotoxic $T$ cells/killer cells, granzyme B, Fas, tumour necrosis factor (TNF), Bax).

Several authors have shown that in local treatment of $\mathrm{SBCC}$ with imiquimod the expression of Fas and FasL (Fas ligand) initially does not change. ${ }^{163.164}$ Berman et al. found that local application of imiquimod does lead to an increased expression of FAS on BCC cells, just as intralesional IFN-a treatment does. ${ }^{165}$ Imiquimod increases the susceptibility of BCC cells for Fas and FasL-mediated apoptosis. Imiquimod does not influence the expression of $p 53$. 
Besides stimulation of cellular defence mechanisms and apoptotic processes imiquimod inhibits angiogenesis. This inhibition is explained by induction of inhibiting cytokines, (interferons, IL-10, IL-12) ${ }^{166}$, local up-regulation of endogenous angiogenic inhibitors, local down-regulation of pro-angiogenic factors and stimulation of apoptosis of the endothelial cell.

In two large phase III studies that evaluated administration of imiquimod five $(n=185)$ and seven $(n=179)$ times a week for six weeks versus vehicle $(n=360)$ within a single superficial $\mathrm{BCC}$, clearance rates were significant higher after 12 weeks for the imiquimod treatment groups than for the vehicle groups (respectively $75 \%$ and $73 \%$ versus $2 \%, p<0,001$ ). ${ }^{167}$ Histopathological clearance rates were $82 \%$ and $79 \%$ versus $3 \%$ in the corresponding treatment groups. In a phase II dose-response trial that evaluated 12 weeks of treatment for single superficial BCCs with imiquimod twice daily, once daily, five times weekly or three times weekly for 12 weeks or vehicle, histological clearance rates six weeks post treatment were significantly higher $(p<0.05)$ for all imiquimod dosing regimens compared with vehicle (respectively $100 \%, 87.1 \%, 80.8 \%$ and $51.7 \%$ vs. $18.8 \%$ ). ${ }^{168}$ Two long-term ongoing multicenter studies investigating the sustained efficacy of imiquimod $5 \%$ cream applied respectively five times a week for six weeks and seven times a week for six weeks of single SBCCs showed clinical clearance rates of $80-90 \%$ after one year of treatment. ${ }^{169.170}$ In addition to SBCCs, imiquimod also demonstrated to be effective in nBCCs. ${ }^{171,172}$ Treatment with imiquimod once daily during respectively 6 and 12 weeks resulted in histopathological clearance rates of $71 \%$ to $76 \% .{ }^{171} \mathrm{An}$ open label study in 15 patients with nodular basal cell carcinoma treated with imiquimod $5 \%$ cream three times a week for 12 weeks showed complete histological clearance rates in $100 \% .{ }^{173}$ An other open label study, showed $74 \%$ clinical effectiveness in a heterogeneous group of BCC after a two years follow-up. ${ }^{174} \mathrm{Tu}-$ mour size appeared to be an independent prognostic variable.

Most common side effects of treatment with $5 \%$ imiquimod cream are local skin reactions as erythema, oedema, vesicles and crusting. The incidence seem to increase with increased frequency of application and there seems to be a positive correlation between the severity of these reactions and the efficacy of treatment. ${ }^{167.168}$ The side-effects however, are generally well tolerated.

In addition to monotherapy, imiquimod can also be used in combination with other surgical treatment modalities as MMS, CE and curettage. ${ }^{175-177}$

\section{Other treatment modalities for basal cell carcinoma}

Besides the treatment modalities discussed in this article, there are other therapies including topical 5-fluorouracil, intralesional interferon, carbon dioxide laser surgery (CO2), pulsed dye laser (PDL), cyclopamine, T4 endonuclease, systemic retinoids, topical retinoids and cisplatin. ${ }^{70.71,73-78,178-182}$ Even ajoene has shown a diminishing effect on $B C C$ in vivo 
via down regulating the expression of the apoptosis-suppressing protein $\mathrm{Bcl}-2 .^{72}$ Large randomised trials with long-term follow-up (>five years) resulting in good efficacy are lacking and therefore these treatment modalities should not be regarded as regular treatments. Further research should be pursued. Table 1.3 portrays these modalities with there key references.

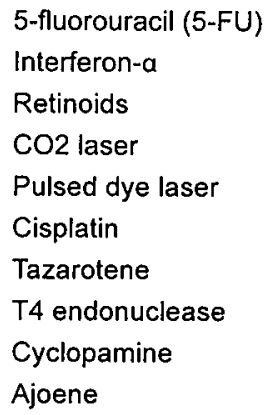

Table 1.3 Other treatment modalities of BCC and their key-references 


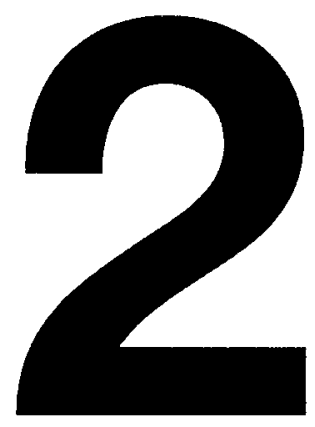

\section{The cutting edge}

\section{Based on the following publications}

Kuijpers DIM, Thissen MRTM, Berretty PJM, Ideler AHLB, Nelemans PJ, Neumann HAM. Surgical excision vs. curettage plus cryosurgery versus in the treatment of basal cell carcinoma in the head and neck area. Results from a randomized controlled trial. In press Dermatol Surg.

Smeets NWJ, Kuijpers DIM, Nelemans PJ, et al. Mohs' micrographic surgery for treatment of basal cell carcinoma of the face--results of a retrospective study and review of the literature. Br J Dermatol 2004;151(1):141-7.

Kuijpers DIM, Smeets NWJ, Krekels GAM, Thissen MRTM. Photodynamic therapy as adjuvant treatment of extensive basal cell carcinoma treated with Mohs' micrographic surgery. Dermatol Surg 2004;30(5):794-8.

Thissen MRTM, Kuijpers DIM, Krekels GAM. Local immune modulator (Imiquimod 5\% cream) as adjuvant treatment after incomplete Mohs' micrographic surgery for large, mixed type basal cell carcinoma. A report of 3 cases. J Drugs in Dermatol. 2006;5:461-4

Kuijpers DIM, Smeets NWJ, Lapière K, Thissen MRTM, Krekels GAM, Neumann HAM. Do systemic antibiotics increase the survival of a full thickness graft on the nose? J Eur Acad Dermatol Venereol 2006;20:1296-1301. 


\section{1}

Surgical excision versus curettage plus cryosurgery in the treatment of basal cell carcinoma of the head and neck. Results from a randomised controlled trial.

Background: Both cryosurgery, with and without prior curettage and surgical excision are common therapeutic strategies for basal cell carcinoma (BCC). However, their efficacy has not yet been compared in a randomised trial.

Objective: To compare the efficacy of curettage plus cryosurgery (C\&C) and surgical excision (SE) in non-aggressive BCC of the head and neck.

Patients \& Methods: A randomised controlled trial was carried out, which included a total of 100 primary BCCs in 88 patients. Tumours were randomly assigned to either $\mathrm{C} \& \mathrm{C}$ $(n=51)$ or $S E(n=49)$. C\&C was performed with a double freeze-thaw cycle after prior curettage of the tumour. SE was performed under standardised conditions with a margin of $3 \mathrm{~mm}$ and with delayed histological examination of the excised tissue.

Results: The median follow-up time was 4.29 years. Recurrences were observed in 13 BCCs, 9 after C\&C (17.6\%) and 4 after SE (8.2\%). The overall five-year recurrence probability was $19.6 \%$ for $C \& C$ and $8.4 \%$ for SE $(p=0.10)$. A Hazard ratio of $2.57(95 \% \mathrm{Cl} 0.79-$ 8.34 ) indicated a putative, but not statistically significant advantage of SE.

Conclusions: These data reflect the outcome of the first randomised controlled trial with long-term follow-up in the treatment of BCC, comparing C\&C with SE. Although no statistically significant difference between the techniques was evident, the observed differences could still be of clinical relevance. Due to the trend towards lower recurrence rates, better cosmetic results and reduced wound healing time, we believe that SE should be preferred to $C \& C$ in the treatment of primary, non-aggressive BCC of the head and neck. 


\section{Introduction}

As the incidence of basal cell carcinoma ( $B C C$ ) is rapidly increasing, the arsenal of treatment modalities is also expanding. When considering the possibilities, different tumourand patient- dependent factors should be taken into account, as well as the experience and skills of the physician. ${ }^{80}$ In all cases, the best available therapy must be applied. Furthermore, functionality, cosmetic results, costs, after-care and possible complications should be evaluated for each patient individually. Therapy for BCC is indreasingly being customised, especially with the growing number of young patients who are mostly well informed. For these younger (and often female) patients, cosmetic results are of greater importance, especially in the head and neck area.

In general, for solid type BCC, surgical excision (SE) is recommended. For aggressive type BCC this is often Mohs' micrographic surgery (MMS). For superficial BCCs, newer therapies such as photodynamic therapy (PDT) en Imiquimod are increasingly being applied with success. ${ }^{157,167}$ To determine the effectiveness of these new, as well as established therapies, evidence-based research are necessary.

Cryosurgery is one of the well-established, older therapies, and is still widely used. The terms cryosurgery and cryotherapy are used interchangeably, with cryosurgery being more accurate in describing the technique in which curettage prior to freezing is often applied in order to define the tumour borders as well as to remove tumour mass. This is also referred to as curettage plus cryosurgery (C\&C) ${ }^{183}$ There is however, no evidence as to whether this curettage affects the efficacy of treatment. ${ }^{184}$ The advantages of cryosurgery lie in the time- and cost-saving aspects. Moreover, cryosurgery is easily performed by experienced hands. Particularly suitable for this treatment are BCCs overlying cartilage in locations such as the outer ear and eyelids. ${ }^{117.118,185,186}$ Cryosurgery is a rather uncontrolled, non selective technique with a major disadvantage being the lack of histopathological evaluation of the tumour margins $A$ search of the literature including a recent review by Kokoska et al, revealed that the number of prospective, comparative studies with a long (5 year) follow-up period concerning this extensively used method is limited and that recurrence rates vary enormously. 95.184

The aim of the present study was to compare C\&C and SE with respect to the efficacy expressed in long-term recurrence rates. In addition, determinants of recurrence in the study population were analysed. 


\section{Materials and Methods}

\section{Study design}

The present study was designed as a randomised, controlled trial (RCT). The primary objective was to compare the efficacy of curettage plus cryosurgery (C\&C) and (SE) in terms of recurrence rates after a 5 year follow-up. Male and female patients older than 18 years of age with previously untreated, primary, histopathologically proven BCCs of the nodular and superficial type were recruited from the outpatient department of dermatology of the Catharina Hospital in Eindhoven, the Netherlands. Patients were recruited in the period between March 1996 and January 1999. Patients were selected according to the following inclusion criteria: superficial or nodular, primary BCC, localised anywhere in the head- and neck area, with a clinical diameter of less than $20 \mathrm{~mm}$. Exclusion criteria comprised: recurrent $\mathrm{BCC}$, histological subtypes other than nodular or superficial, tumours larger than 20 $\mathrm{mm}$ in diameter, patients with contra-indications to either surgery or cryosurgery, and with the presence of 5 or more BCCs. After the patients had given informed consent, the BCCs were randomly assigned to either SE or C\&C. Randomisation was performed centrally by telephone by a person not involved in the trial, using a computer-generated random allocation scheme. Recurring tumours were treated surgically, either by SE or by Mohs' micrographic surgery.

\section{Treatment methods}

\section{Curettage plus Cryosurgery (C\&C)}

Under local infiltration anaesthesia with $1 \%$ lidocaine with epinephrine, curettage was performed using Volkmann's sharp curettes. After curettage, hemostasis was achieved using $65 \%$ feri-ferro-chloride solution.

A liquid nitrogen spray unit (Frigitronics CS-76, Shelton Conn., USA, Figure 2.1) was used to freeze the tissue under an operating pressure of 8 psi. Freezing was carried out in two cycles of 20 seconds each, with a halo thaw time in between of 60 seconds. ${ }^{115.120}$ All tumours were treated with the open cone spray technique using a neoprene cone with a wall thickness of $2 \mathrm{~mm}$. The cone was modelled to a proper shape so as to enclose the tumour and allow a macroscopic free margin of $5 \mathrm{~mm}$ around the tumour. If the tumour and $5 \mathrm{~mm}$ margin was too large to be enclosed by the cone, freezing was performed in fractions. After freezing, the treated area was covered with petrolatum dressings until the entire defect showed re-epithelialisation. In the C\&C group, evaluation of completeness of treatment was only possible clinically. Treated areas with no visible tumour tissue remaining during the first and second wound control visits were considered completely treated. 


\section{Surgical excision (SE)}

Anaesthesia was performed by local injection of $1 \%$ lidocaine with epinephrine. The tumour including a $3 \mathrm{~mm}$ margin, which was carefully drawn onto the skin, was excised. ${ }^{82}$ Closure of the defect was either primary by suturing in different layers, or by transposition or transplantation techniques depending on the localisation and size of the final defect. Routine histological examination of haematoxylin-eosin stained sections of the lateral and deep margins was performed in the first week to determine whether or not the excision was complete, taking into account the shortcomings of this so-called bread loaf technique ${ }^{88}$ In case of positive margins, one or more re-excisions were performed until all margins were free of tumour.

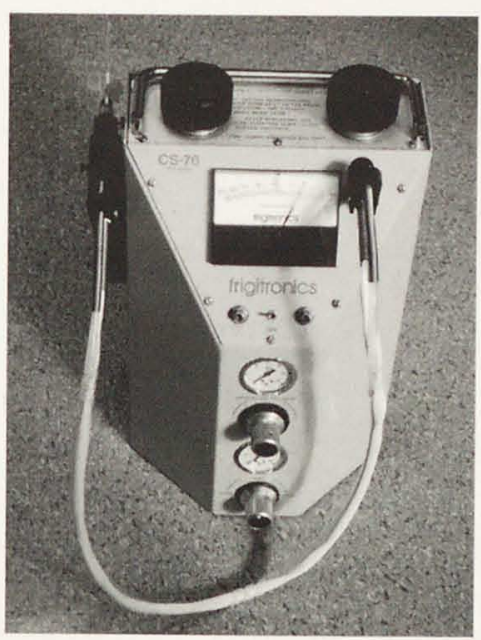

Figure 2.1 Liquid nitrogen spray unit (Frigitronics CS-76, Shelton Conn., USA)

\section{Follow-up}

Follow-up visits took place at day 7 (removal of the stitches, inspection of the cryosurgery wound) and at day 30. According to the recommendations of the Dutch Society of Dermatology and Venereology, all patients were subsequently examined for recurrent tumours at 3, 6, 9, 12, 18 months, and after 2, 3, 4 and five years. If necessary, patients had extra consultations for control of their wound in the first month. A recurrent tumour was defined as a histologically confirmed BCC within $5 \mathrm{~mm}$ from the scar. Due to the large inter-treatment variation in execution, blinding of the patients as well as the physicians was not possible. However, dermatologists other than the treating research physician conducted the oncological follow-up visits, when possible. 


\section{Statistical analysis}

Data were analysed using SPSS-PC for Windows version 12.0. Assuming that an estimated $95 \%$ of lesions would show complete response to SE, and that the response to $\mathrm{C} \& \mathrm{C}$ would be the same in this study population, it was estimated that approximately 50 lesions per group would be required to demonstrate with $95 \%$ confidence and a power of $90 \%$ that C\&C was no more than $15 \%$ inferior to SE. The $15 \%$ difference was agreed upon as clinically relevant by the dermatologists who participated in this study. The analysis was performed according to the intention to treat principle. The five-year cumulative probability of recurrent BCC in both treatment groups was compared using a Kaplan-Meier survival analysis and the log rank test, which computes the weighted difference between the observed and expected number of recurrences at each of the time points. ${ }^{187}$ Patients lost to follow-up or who died during the trial were censored on the date of last examination. A p-value of 0.05 or less was considered statistically significant. A Cox regression model was used to assess the independent effect of treatment modality and other predictors on the recurrence rate. ${ }^{188}$ These independent effects are expressed in terms of hazard ratios (HR) and corresponding $95 \%$ confidence intervals $(95 \% \mathrm{Cl})$.

\section{Results}

\section{Patient and tumour characteristics}

A total of 88 patients with 100 BCCs were treated from June 1996 until March 1999. More men than women were treated $(57 \%, n=50$ vs. $43 \%, n=38)$. The mean age of the patients at the time of treatment was 67 years of age (median 67, range 34-92). Most of the BCCs were located on the forehead or temple $(27 \%, n=27)$, on the nose or para-nasal zone $(22 \%, n=22)$ and on the cheeks/chin region (16\%, $n=16)$ (Figure 2.2). Six patients had two BCCs included, one patient had three and one patient had four tumours. The remaining 92 patients all had one single $B C C$ included. The tumours had a mean size of $5.4 \mathrm{~mm}$ (SD 2.8 , range 3-20) with respect to its longest diameter. Ninety-six tumours had a histological nodular subtype, whereas four tumours were superficial. The clinical characteristics of the BCCs (table 2.1) before treatment were similar in the two groups.
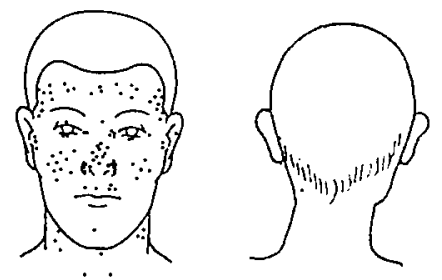

Figure 2.2 Distribution of tumours 


\begin{tabular}{|c|c|c|c|}
\hline & \multicolumn{3}{|c|}{ Number $(\%)$} \\
\hline & $\begin{array}{l}\text { Curettage- } \\
\text { Cryosurgery } \\
(n=51)\end{array}$ & $\begin{array}{l}\text { Surgical } \\
\text { excision } \\
(n=49)\end{array}$ & $\begin{array}{l}\text { All tumours } \\
(n=100)\end{array}$ \\
\hline \multicolumn{4}{|c|}{ Histopathological type } \\
\hline Nodular & 51 & 45 & 96 \\
\hline Superficial & 0 & 4 & 4 \\
\hline \multicolumn{4}{|l|}{ Localisation } \\
\hline Forehead/temple & $15(29)$ & $12(25)$ & $27(27)$ \\
\hline Cheek/chin & $9(18)$ & $7(14)$ & $16(16)$ \\
\hline Nose/perinasal & $11(22)$ & $11(22)$ & $22(22)$ \\
\hline Lips/mouth & $2(4)$ & $3(6)$ & $5(5)$ \\
\hline Periocular & $6(12)$ & $7(14)$ & $13(13)$ \\
\hline Ears/periauricular & $4(8)$ & $3(6)$ & $7(7)$ \\
\hline Neck & $3(6)$ & $5(10)$ & $8(8)$ \\
\hline Chest/back & $1(2)$ & $1(2)$ & $2(2)$ \\
\hline \multicolumn{4}{|c|}{ Size (mean longest diameter) } \\
\hline Mean (SD) & $5.4 \mathrm{~mm}(2.9)$ & $5.3 \mathrm{~mm}(2.6)$ & $5.4 \mathrm{~mm}(3.8)$ \\
\hline $1-5 \mathrm{~mm}$ & $37(73)$ & $37(76)$ & $74(74)$ \\
\hline $6-10 \mathrm{~mm}$ & $13(26)$ & $11(22)$ & $24(24)$ \\
\hline $11-15 \mathrm{~mm}$ & 0 & $1(2)$ & $1(1)$ \\
\hline $16-20 \mathrm{~mm}$ & $1(2)$ & 0 & $1(1)$ \\
\hline
\end{tabular}

Table 2.1 Tumour characteristics

\section{Treatments}

Forty-nine BCCs were treated by SE and 51 by C\&C. All tumours were treated according to the treatment modality to which they were assigned. In the excision group, $46 \mathrm{BCCs}$ were completely treated in the first treatment. Three BCCs were excised incompletely. One patient with one tumour that was still incompletely treated after two excisions was referred to a university hospital for MMS.

None of the tumours treated by C\&C had clinically residual tumour during the first month, therefore such tumours were considered to be treated completely.

\section{Follow-up}

The median follow-up time was 4.29 years (range $0-5$ ), with 85 tumours ( $85 \%$ ) having a follow-up period of five years. Thirteen patients with fifteen tumours were lost during followup due to death by unrelated cause (9), illness (1), or unknown reason (3). The follow-up duration differed between the two groups: in the excision group, 2 tumours (4\%) were lost to follow-up (both due to death) compared to 13 tumours (25\%) in the cryosurgery group. The difference between the two treatment groups seems to be a coincidence as there were a high number of unrelated deaths $(9 / 13,69 \%)$.

The majority of recurrences after $\mathrm{C} \& \mathrm{C}$ occurred within three years. In the fifth year, the number of recurrences did not increase in comparison to that after four years in either of the groups. 


\section{Recurrence rates}

In total, 13 BCCs recurred, 9 of which recurred after C\&C and 4 after SE. If we exclude all patients that were not followed for the whole period of five years, the recurrence rates would be $23.7 \%(9 / 38)$ and $8.5 \%$ (4/47). The overall five-year recurrence probability, calculated with Kaplan-Meier survival analysis, which makes fuller use of all available data, is $19.6 \%$ for $C \& C$ and $8.4 \%$ for SE $(p=0.10)$ (Figure 2.4). In Table 2.2, the cumulative numbers of recurrences per year are demonstrated for both treatment groups.

Using the Cox regression model, without adjustment for prognostic factors, the HR is 2.57 (95\% $\mathrm{Cl} 0.79-8.34$ ), favouring SE. The HR with adjustment for age, gender, size, and localisation indicates that the risk of recurrence after $C \& C$ is more than two times higher than after SE (HR=2.38,95\% Cl: 0.72-7.84). Localisation on and around the nose and a larger size of the primary BCC (>5 mm) are also associated with a two-fold higher risk of recurrence ( $H R=2.68$ and $H R=2.03$, respectively). In Figure 2.3 the anatomical distribution of the recurrent tumours is highlighted. Age of older than 70 years and being female seem to be associated with a somewhat lower risk of recurrence ( $H R=0.82$ and $H R=0.65$, respectively). According to the $95 \% \mathrm{Cl}$, none of these results are statistically significant. The results of this analysis are summarised in Table 2.3.

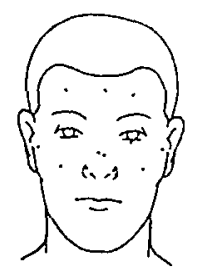

Figure 2.3 Distribution of recurrent tumours

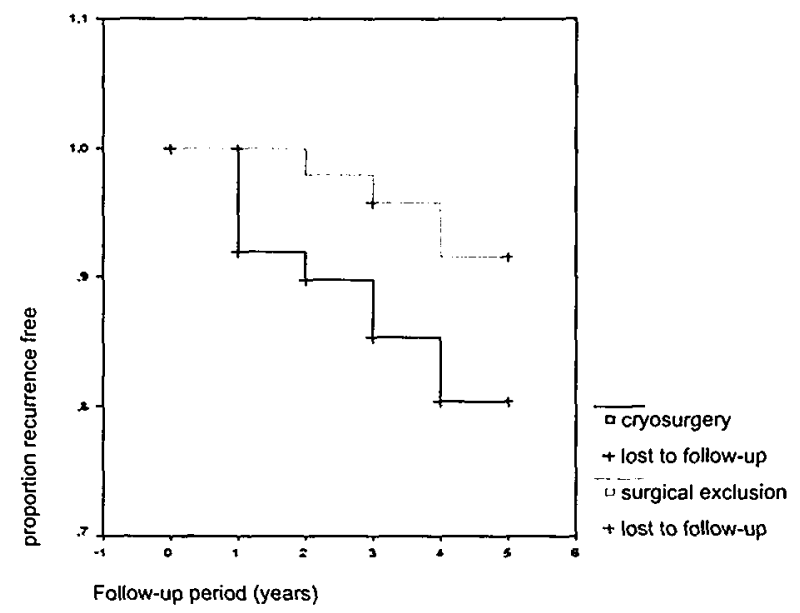

Figure 2.4 Kaplan-Meier survival analysis 


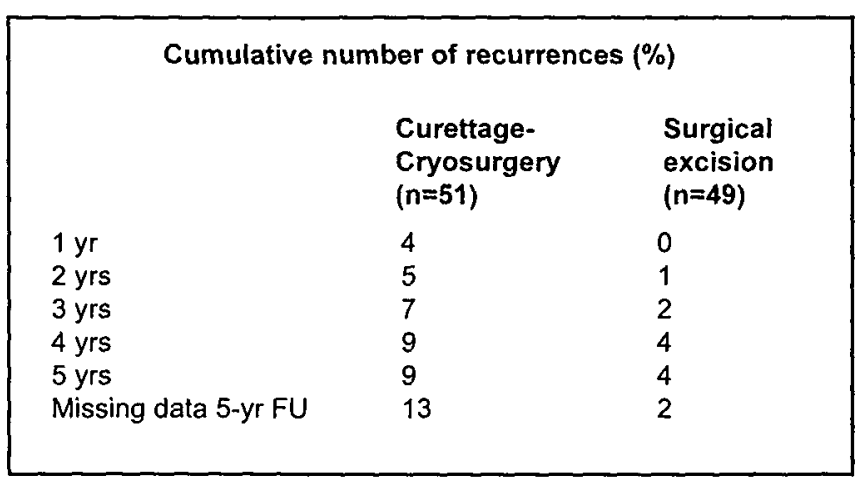

Table 2.2 Cumulative number of recurrences

\begin{tabular}{|c|c|c|}
\hline \multicolumn{3}{|c|}{ Cox regression analysis } \\
\hline & $\begin{array}{l}\text { Adjusted } \\
\text { Hazard ratio }\end{array}$ & $\begin{array}{l}95 \% \text { confidence } \\
\text { intervals }\end{array}$ \\
\hline Treatment modality & & \\
\hline $\begin{array}{l}\text { C\&C vs. SE } \\
\text { Localisation }\end{array}$ & 2.38 & $0.72-7.84$ \\
\hline $\begin{array}{l}\text { Nasal/perinasal vs. non-nasal } \\
\text { Size }\end{array}$ & 2.68 & $0.85-8.46$ \\
\hline $\begin{array}{l}>5 \mathrm{~mm} \text { vs. } \leq 5 \mathrm{~mm} \\
\text { Age }\end{array}$ & 2.03 & $0.63-6.55$ \\
\hline$>70$ yrs vs. $\leq 70$ yrs & 0.82 & $0.27-2.53$ \\
\hline $\begin{array}{l}\text { Sex } \\
\text { Female vs. male }\end{array}$ & 0.65 & $0.19-2.25$ \\
\hline
\end{tabular}

Table 2.3 Prognostic variables

If the hazard ratio is associated with a variable 1 , the variable does not influence probability of recurrence. A hazard ratio larger than 1 indicates that patients with the characteristic have an increased risk of recurrence compared to patients without the characteristic. A hazard ratio lower than 1 indicates a decreased risk of recurrence.

\section{Complications}

As with regular treatment, all side effects and adverse events as a result of treatment were documented, as were the extra visits and necessary measurements.

Secondary wound infection, treated with systemic antibiotics was seen in three cases within the C\&C group (3/51=5.9\%). In the SE group, systemic antibiotics were necessary for secondary wound infection in four patients in the first and second week after treatment. Serious complications like ectropion, nerve damage, or damage to the lacrimal duct were not observed after C\&C or after SE. 


\section{Discussion}

To our knowledge, this is the first randomised controlled trial with a designed 5 year followup in the treatment of the most common skin cancer in the Caucasian population, in which C\&C is compared to SE.

A difference between the recurrence rates of C\&C and SE (19.6\% vs. $8.4 \%)$ was found not to be statistically significant according to our analysis $(p=0.10)$. Most probably, the lack of statistical significance is due to the relatively small sample size, which is the limitation of this study. However, a clinically relevant difference in recurrence rate cannot be excluded. Based on changes in the preferred treatment of BCC, and given the expansion of treatment possibilities, it is unlikely that a future sufficiently large clinical trial would be conducted to arrive at a definitive conclusion. Besides the fact that a clinically relevant higher risk of recurrence exists after $\mathrm{C \& C}$, other arguments for the use of SE in the treatment of $\mathrm{BCCs}$ are discussed here.

Due to the histological control after SE and subsequent re-excision when necessary, the risk of remaining small residual tumour buds declines, leading to a better prognosis. This advantage is absent in all treatment modalities without histological control, as in C\&C.

Cryosurgery is time-saving, relatively inexpensive and easy to perform by experienced hands. A disadvantage is the lack of histological control considering the completeness of this treatment, and the relative long period of wound healing. Contraindication for cryosurgery, as found in literature, is the location, such as on the central part of the face, hairy scalp and eyebrows, which poses an increased risk for scarring alopecia, and the lower legs and feet due to the risk of ulceration. In addition, tumour related characteristics, such as size, aggressive type, recurrency, fixation to bone and deep invasion are considered relative contraindications.

The recurrence rates in this study are comparable with those found in literature. In their review Kokoska et al. identified four randomised, comparative studies regarding cryosurgery, including the one year results of the present study. ${ }^{184}$ Recurrence rates vary from $4.2 \%$ to $39 \%$ after a two year follow-up. ${ }^{102,119-121.184}$ Thirteen non-controlled, prospective studies with recurrence rates varying from $0 \%$ to $21 \%$ were found. Four of these prospective and one retrospective study had a long follow-up (five years). ${ }^{14.116-118.185}$ One article reported retrospectively 5 recurrences in 522 BCCs after five years, without mentioning loss to follow-up. ${ }^{122}$

Regarding surgical excision, only two randomised controlled trials were identified. In one study comparing radiotherapy to excision, 1 out of 174 excised BCCs recurred after four years of follow-up. ${ }^{101,189}$ A large, randomised controlled trial comparing Mohs' micrographic surgery to surgical excision in facial BCC revealed a recurrence rate of $3 \%$ for primary tumours during a 30-month follow-up period. ${ }^{100}$

Specifically for SE of primary BCCs, prospective studies with a long follow-up, make note 
of cumulative five-year recurrence rates varying from $1.7 \%$ to $8.0 \%$. The crude recurrence rates in these studies vary from $1.1 \%$ to $2.9 \% .64 .94 .99 .125$ The recurrence rate for SE found in the present study falls at the upper limit of these rates. We have tried to find explanations for this in tumour localisation since we had a high proportion of tumours located centrally on the face, which lead to a relatively high recurrence rate of $8.4 \%$. This finding is described as well by Silverman et al., and other authors, who found the H-zone, and more specifically, noses and ears to be high-risk sites in the head and neck area. ${ }^{94.190} \mathrm{De}$ terminants of the recurrence of primary, completely treated BCC as found in literature are, besides the above mentioned localisations, aggressive histopathological subtype (morpheic, micronodular, BCC with squamous differentiation, perineural invasion), large size (>20mm), young age, and male gender. ${ }^{61,191.192}$

Studies reporting recurrence rates based on a follow-up shorter than five years can be expected to underestimate the true recurrence rate. Rowe et al. calculated that the average five-years recurrence rate is more than twice that found in short-term (two-year) follow-up studies. ${ }^{96}$ Of the recurrences after treatment of primary BCC (independent of the treatment modality), one third of recurrences occur in the first year, $50 \%$ within the first two years and two-thirds within a three-year follow-up. Eighteen percent occurs between the fifth and the tenth year of follow-up. Other retrospective analyses resulted in comparable figures. ${ }^{97}$

It is therefore possible that the recurrence rates calculated in our study would further increase with time. A longer follow-up would be helpful in detecting these recurrences, but in general, as stated in Dutch guidelines, the proposed follow-up for single BCCs is five years. Lifelong check-ups for patients with a single BCC leads to unwanted and unnecessary health costs and are therefore generally not advised.

Thissen et al. performed a comparison of cosmetic results between cryosurgery and SE after one year by using results of the treated population of the randomised trial described here. ${ }^{102}$ In general, cosmetic results after surgical excision were better than after cryosurgery. The cosmetic results were not re-evaluated after five years because no further improvement was expected after the first year, as the scar is then considered to be inactive and definite. More studies confirm the good to excellent cosmetic outcome following surgical excision and good cosmetic outcome following C\&C. ${ }^{94.119 .193}$

Only a few RCTs concerning treatment of BCC are published and the non-controlled and retrospective studies that have been performed, are difficult to compare because of differences in tumour type, number of patients lost in follow-up, size, previous treatments, execution of treatment, duration of follow-up, statistical analysis, etcetera. In general, more comparative studies with long follow-up regarding therapies for $\mathrm{BCC}$ are needed to determine the best patient-based strategy in the treatment of the most common skin cancer in the Caucasian population. 


\section{Conclusion}

To the best of our knowledge, this is the first RCT comparing C\&C to SE with a five-year follow-up in the treatment of BCC. A clinically important difference between the recurrence rates of SE and C\&C seems to exist, although this difference did not reach statistical significance. Our data show that rather high recurrence rates are obtained after cryosurgery (19.6\%) for primary, non-aggressive BCCs. These recurrence rates are comparable or even higher than those found in literature. The efficacy of surgical excision in this study population is better with a recurrence rate of $8.4 \%$. This recurrence rate is comparable to that found in other studies, but still relatively high. This is possibly due to the high number of tumours localised centrally on the face. As our data are based on 100 tumours, a larger number of samples are necessary to achieve statistical significance, thereby confirming trend towards the superiority of SE. Large size (more than $5 \mathrm{~mm}$ ) and nasal/perinasal localisation seem important prognostic factors for recurrence.

Though C\&C displays some advantages (easy to perform, low initial costs), it is questionable whether these advantages of $C \& C$ outweigh these higher recurrence rates, shorter wound healing and inferior cosmetic results. In our opinion, surgical excision is preferable to curettage plus cryosurgery in the treatment of primary non-aggressive $B C C$ in the head and neck area. 


\section{Mohs' micrographic surgery for treatment of basal cell carcinoma of the face; review of the literature and results of a retrospective study}

Background: The incidence of skin cancer and especially basal cell carcinoma (BCC) has increased the last decennia and is still rising. Many treatment modalities can be used to treat $\mathrm{BCC}$, with surgical excision being most frequently used. Mohs' micrographic surgery (MMS) is an advanced excision technique which is often used to treat BCC in the USA. In Europe it is practised at a lower scale.

Objective: The goal of this article was to evaluate the efficiency of MMS in the treatment of facial BCC.

Methods: In a retrospective study recurrence rates after treatment of facial BCC by MMS were estimated by reviewing the records of all patients with BCCs (720 BCCs) treated by MMS at our department from April 1992 until December 1999.

Results: The five-year recurrence rates estimated from this study were $3.2 \%$ for primary $\mathrm{BCC}$ and $6.7 \%$ for recurrent BCC. Prognostic factors for recurrence are: an aggressive histopathological subtype, more than four Mohs' stages, defect size and recurrent $B C C$. Conclusion:Based on the fact that MMS provides the lowest recurrence rates, it is the treatment of first choice in primary facial $B C C s$ with an aggressive histopathologic subtype and in recurrent $\mathrm{BCCs}$ in the face. 


\section{Introduction}

Basal cell carcinoma (BCC) is the most common cancer in Caucasians, occurring in one out of every five-six persons in a lifetime and the incidence is still rising every year. ${ }^{2,194.195}$ For treatment of BCCs multiple modalities can be used, with surgical excision being most frequently used. ${ }^{196.197}$

Mohs' micrographic surgery (MMS) was first described by Dr. Frederic E. Mohs in $1941 .^{198}$ His fixed tissue technique (chemosurgery) was based on the principle that when zinc chloride was applied to tissue, the tissue was fixed and the tumour could be horizontally shaved off, after which $100 \%$ of the resection margins could be examined microscopically. Areas of cancer found with the microscopic examination were located on maps, drawn on both paper and on the tissue itself. This way only there where residual tumour was located a re-excision was performed. The technique was refined and is nowadays performed under local anaesthesia without fixation of the tumour in vivo. The horizontally cut frozen sections and the graphically mapping remained. Mohs named his technique chemosurgery, because of the chemical interaction with zinc chloride and human tissue. Nowadays it is renamed micrographic surgery, because it combines microscopic control of tumour and graphical registration for indicating the remaining tumour and further surgery. ${ }^{199}$

In the USA, MMS is used for approximately $30 \%$ of all BCCs. ${ }^{200}$ In Europe, MMS was introduced in the early 1980s and is still practised at a lower scale, mostly in (referral) university hospitals. ${ }^{201}$ Because there are less facilities to practice MMS, only a very select group of skin cancers is treated by it. ${ }^{106.201}$ MMS has been introduced in 1992 in our university clinic and during the first years it was the only hospital in the Netherlands were MMS was practised.

The goal of this article was to evaluate the efficiency of MMS in the treatment of BCC of the face. We therefore retrospectively analysed the five-year follow-up results of the cases treated in the University Hospital of Maastricht and compare these with literature results.

\section{Methods}

At the University Hospital of Maastricht, all BCCs treated with MMS from April 1992 until December 1999 were included in the clinical analysis. The analysis was performed in June 2002 so the majority of the patients with a BCC had completed a five-year follow-up period. Inclusion criteria for treatment with MMS in our department in this period were;

- BCC located in the H-zone of the face (Fig 2.5), and/ or,

- BCC of a, biopsy proven, aggressive histopathologic subtype (morphea, micronodular and $\mathrm{BCC}$ with squamous differentiation) located in the face, and/ or,

- large $B C C(>2 \mathrm{~cm}$ ) (of the face), and/ or,

- recurrent BCC (located in the face).

In MMS, the tumour with a small margin of $2 \mathrm{~mm}$ was excised at an angle of approximately 
$45^{\circ}$ as described before. ${ }^{7}$ The specimen was then processed into horizontal frozen sections in such a way that theoretically $100 \%$ of the margins could be viewed. The slides were examined by both a dermopathologist and the Mohs' dermatologist. In case of positive resection margins, a second excision (Mohs' stage) was performed. This procedure was repeated until complete tumour removal was achieved. In most cases, the defect was reconstructed immediately postoperatively. All patients entered an oncological follow-up system for five years at the University Hospital of Maastricht or at their own (referring) dermatologist.

All data were retrospectively collected by reviewing patient files in the University Hospital of Maastricht as well as in referring non-academic clinics in the region. Data were collected on: occurrence of a recurrence, length of follow-up, reasons for not completing follow-up and prognostic factors, such as localisation, size, histological subtype, type of previous therapy, previous BCC, defect size and number of Mohs' stages. A recurrent BCC was defined as a histologically confirmed BCC within $5 \mathrm{~mm}$ from a scar. In approximately 150 patients the follow-up was extended by a questionnaire among general practitioners which inquired about the date of the last control visit and whether a recurrence had occurred.
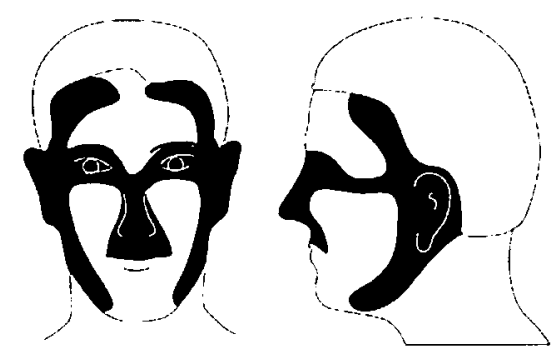

Figure 2.5 The H-zone

\section{Statistical analysis}

Data were analysed using SPSS-PC for Windows. The five-year cumulative probability of recurrent BCC after treatment by MMS was estimated by using a Kaplan-Meier survival analysis. The difference in time until a recurrence after primary and recurrent $B C C$ was tested by the non-parametric Mann-Whitney test. In order to evaluate which factors are prognostic for a recurrent BCC after treatment, patients were classified into groups with or without the specific prognostic factors. Survival functions across groups of patients were compared using the log rank test, which computes the weighted difference between the observed and expected number of recurrences at each of the time points. A p-value of 0.05 or less was considered to indicate statistical significance. 


\section{Results}

A total of 633 patients with 737 BCCs were treated from April 1992 until December 1999. Eleven patients with eleven BCCs were excluded because these BCCs were not located in the head/ neck area. Two patients with in total six BCCs, known with nevoid basal cell carcinoma syndrome (NBCCS), were also excluded. So 620 patients with 720 BCCs remained. More men than women were treated $(55.6 \%, n=345$ vs. $44.4 \%, n=275)$. The mean age of the patients was 65 years (median 67 years, range 22-94). For most patients $(70.2 \%, n=427)$, this BCC was the first skin malignancy. Sixty-six percent $(n=404)$ of the patients was referred from other hospitals.

The majority of the BCCs was located on the nose $(30.3 \%, n=219)$ and on the forehead/ temporal zone $(23.1 \%, n=167$, Fig 2.6). The tumours had a mean size of $15.78 \mathrm{~mm}$ (SD $12.40)$, with $21.5 \%(n=139)$ having a diameter of more than $20 \mathrm{~mm}$. Other tumour characteristics are mentioned in Table 2.4.

Only $24.7 \%$ ( $n=177$ ) of all BCCs was eradicated in one Mohs' stage (Fig 2.7). In 46\% $(n=331)$ of cases two stages were needed to achieve tumour free resection borders. The mean defect size was $28.55 \mathrm{~mm}$, with $32 \%(n=203)$ having a defect size less than $2 \mathrm{~cm}$ and $22 \%(n=136)$ a size more than $4 \mathrm{~cm}$. The procedure took place under local anaesthesia in $85.5 \%(n=616)$ and in $14 \%(n=101)$ general anaesthesia was required. In two cases $(0.4 \%)$, the procedure was started under local anaesthesia and continued under general anaesthesia because of the unexpected extension of the tumour. The median follow-up was 3.57 years (range: $0.0-9.61$ ) with 292 tumours (37\%) having a follow-up period of five years or more. Recurrences occurred in 27 of 720 BCCs $(3.8 \%$, Table 2.5$) ; 11(2.4 \%)$ after treatment of a primary $\mathrm{BCC}$ and $16(6.3 \%)$ after treatment of a recurrent BCC. Two patients had a BCC that recurred twice after treatment with MMS. The five-year cumulative probability of recurrence after treatment by MMS was estimated by using Kaplan-Meier survival analysis (Fig 2.8). The overall five-year recurrence rate is $4.5 \% ; 3.2 \%$ for primary $\mathrm{BCC}$ and $6.7 \%$ for recurrent $\mathrm{BCC}$. The difference between primary and recurrent $\mathrm{BCC}$ is statistically significant $(p=0.0233)$. The seven-year recurrence rate is for primary BCC the same as the five-year recurrence rate. However, the seven-year recurrence rate for a recurrent $\mathrm{BCC}$ is $15.8 \%$, which is much higher than the five-year recurrence rate. The median time for a recurrent $B C C$ to appear after treatment of a primary $B C C$ is 1.92 years (range 0.52-3.41), while after treatment of a recurrent $B C C$ this median time is 2.51 years (range 0.44-6.62). So, a recurrence after treatment of a recurrent $\mathrm{BCC}$ develops later and in a high percentage of cases after five years of follow-up, but this difference is not statistically significant (Mann-Whitney, $p=0.169$ ). Of the 27 recurrences in the total study group, most recurrences date from the early treatment years. Of all BCCs treated in $19926.7 \%$ recurred, in $199311.9 \%$ recurred, in $199412.0 \%$ recurred, in $19956.7 \%$ recurred and in $19962.5 \%$ recurred. There is a significant difference in recurrence rates between the first 
two years (1992-1993) and the last two years (1998-1999, $p=0.0012)$. Comparing survival curves of groups of patients with and without relevant prognostic factors (Table 2.6), we note that non-aggressive BCCs (nodular and superficial) have a significant lower five-year cumulative probability to recur than aggressive type BCCs; $2.9 \%$ versus $5.8 \%(p=0.0150$ ). With an increasing number of Mohs' stages, the five-year cumulative probability of recurrence increases significantly to a probability of $9.0 \%$ in case of four or more Mohs' stages $(p=0.0013)$. If the defect after MMS exceeds $4 \mathrm{~cm}$ in diameter, there is also a significant higher five-year probability of recurrence $(p=0.0027)$ compared with smaller defects. Large BCCs (> 3cm) have a higher five-year probability to recur $(7.8 \%)$ than smaller $(<1 \mathrm{~cm})$ tumours $(3.7 \%)$ but this difference is not statistically significant. We found that the localisation of the BCC, previous therapy or a history of a previous BCC had no prognostic significance.

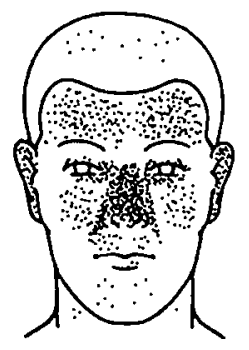

Figure 2.6 Distribution of tumours

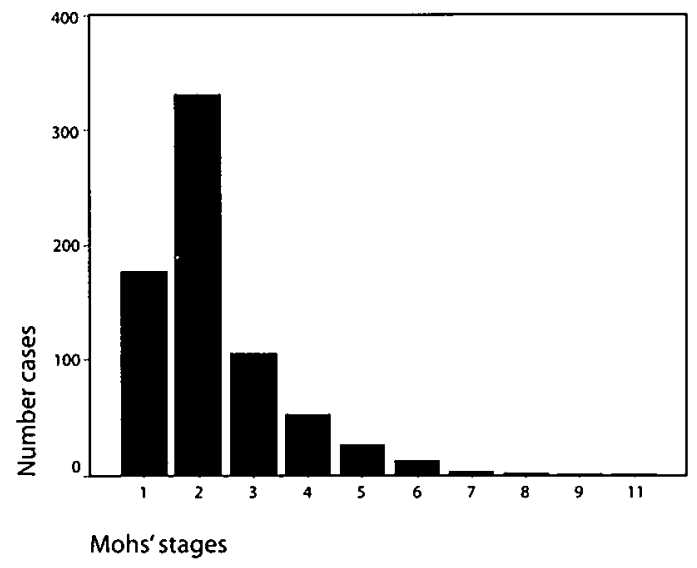

Figure 2.7 Number tumours per Mohs' stage 


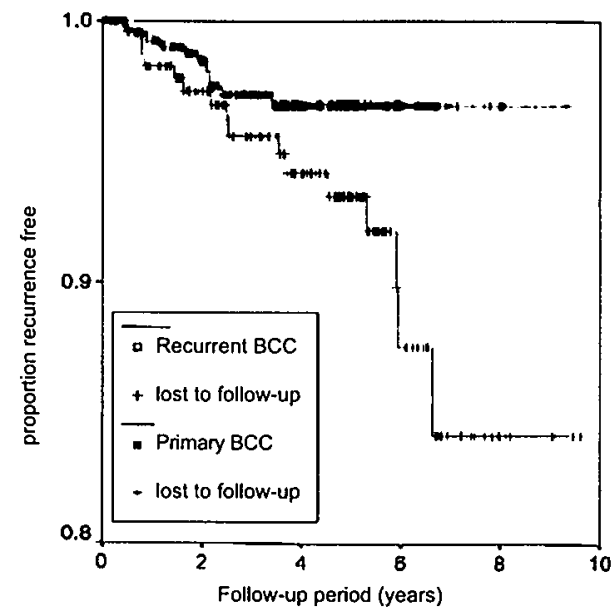

Figure 2.8. Kaplan-Meier survival analysis

Size $(\mathrm{mm})$

- $\leq 10$

- $11-20$

- 21-30

- $31>$

Missing

Total

\section{Histological type}

- non-aggressive

- aggressive

- missing

Total

Primary/ recurrence

- Primary

- 1e recurrence

- 2e recurrence

- 3e or more recurrence

- Incomplete primary

- Incomplete recurrence

- Missing

Total

Previous therapy (recurrence)

- Surgical excision

- Radiotherapy

- Cryosurgery

- Curettage + electrodessication

- MMS

- Others

- Several

Total
Number (\%)

294 (45.4)

214 (33.1)

$84(13.0)$

$55(8.5)$

73

720

325 (45.3)

393 (54.7)

2

720

$365(50.8)$

$142(19.7)$

$46(6.4)$

$38(5.3)$

$100(13.9)$

$28(3.9)$

1

720

$115(45.3)$

$13(5.1)$

$44(17.3)$

$9(3.5)$

$12(4.7)$

$16(6.3)$

45 (17.7)

254
Mean (SD)

$15.78(12.40)$ 


\begin{tabular}{|lll|}
\hline Recurrence & Number (\%) & Mean (SD) \\
- No & $693(96.2)$ & \\
$-\quad$ Yes & $27(3.8)$ & \\
Total & 720 & $3.53(2.10)$ \\
Follow-up time & & \\
-5 or more & $272(37.6)$ & \\
-4 & $94(13.1)$ & \\
-3 & $86(11.9)$ \\
-2 & $133(18.5)$ \\
-1 & $75(10.4)$ \\
- less than 1 year & $60(8.3)$ \\
Total & 720 \\
Reason non-follow-up & $90(19.9)$ \\
- died 1 year after last FU & $4(0.9)$ \\
- illness & $359(79.2)$ \\
- unknown & 453 \\
Total & \\
\hline
\end{tabular}

Table 2.5 Recurrence and follow-up 


\begin{tabular}{|c|c|c|c|}
\hline \multicolumn{2}{|l|}{ Localisation } & $(\%)^{*}$ & test, $p=$ \\
\hline - forehead/temporal & 156 & $10(9.1)$ & \\
\hline - peri-ocular & 73 & $1(1.7)$ & \\
\hline - nose & 212 & $6(2.3)$ & \\
\hline - perinasal & 76 & $4(7.6)$ & \\
\hline - cheek & 63 & $3(3.7)$ & \\
\hline - ear/ peri-auricular & 59 & $3(3.5)$ & \\
\hline - upper lip & 20 & - & \\
\hline - lips & 2 & - & \\
\hline - chin & 10 & - & \\
\hline - vertex & 14 & - & \\
\hline - neck & 4 & - & \\
\hline Total & 689 & $27(4.5)$ & 0.7383 \\
\hline \multicolumn{4}{|l|}{ Size (mm) } \\
\hline$-<20 \mathrm{~mm}$ & 437 & $13(3.7)$ & \\
\hline$-\quad \geq 20 \mathrm{~mm}$ & 186 & $11(7.8)$ & \\
\hline Total & 623 & $24(4.5)$ & 0.0648 \\
\hline \multicolumn{4}{|l|}{ Histological type } \\
\hline - non-aggressive & 319 & $6(2.9)$ & \\
\hline - aggressive & 372 & $21(5.8)$ & \\
\hline Total & 691 & $27(4.5)$ & 0.0150 \\
\hline \multicolumn{4}{|l|}{ Previous therapy (recurrence) } \\
\hline - Surgical excision & 106 & $9(8.7)$ & \\
\hline - Radiotherapy & 13 & $0(0.0)$ & \\
\hline - Cryosurgery & 43 & $1(2.9)$ & \\
\hline - Curettage + electrodessication & 8 & $1(12.5)$ & \\
\hline - MMS & 11 & $1(0.0)$ & \\
\hline - Several & 41 & $4(10.7)$ & \\
\hline - Others & 16 & $0(0.0)$ & \\
\hline Total & 238 & $16(6.7)$ & 0.5034 \\
\hline \multicolumn{4}{|l|}{ Previous BCC } \\
\hline - no BCC & 428 & $20(5.3)$ & \\
\hline - previous BCC & 239 & $7(3.7)$ & \\
\hline - Total & 667 & $27(4.5)$ & 0.3195 \\
\hline \multicolumn{4}{|l|}{ Mean defect size (mm) } \\
\hline$-\leq 20$ & 198 & $5(2.4)$ & \\
\hline$-20-40$ & 275 & $9(3.0)$ & \\
\hline$-\geq 40$ & 123 & $12(12.7)$ & \\
\hline Total & 596 & $26(4.5)$ & 0.0027 \\
\hline \multicolumn{4}{|l|}{ Mohs'stages } \\
\hline-1 & 169 & $8(4.5)$ & \\
\hline-2 & 328 & $3(0.9)$ & \\
\hline-3 & 101 & $5(4.7)$ & \\
\hline - 4 or more rounds & 91 & $11(10.8)$ & \\
\hline Total & 689 & $27(3.8)$ & 0.0013 \\
\hline
\end{tabular}

\section{Recurrence Recurrences} free

Number Number Log rank

$(\%)^{\star} \quad$ test, $p=$

- ear/ peri-auricular

- upper lip

- lips

- chin

- vertex

Size $(\mathrm{mm})$

- $<20 \mathrm{~mm}$

- $\geq 20 \mathrm{~mm}$

Total

Histological type

- non-aggressive

Total

Previous therapy (recurrence)

- Surgical excision

- Radiotherapy

- Cryosurgery

- Curettage + electrodessication

MMS

- Others

Total

Previous BCC

- no BCC

previous BCC

- Total

$-\leq 20$

- $20-40$

275

123

$12(12.7)$

$8(4.5)$

$3(0.9)$

$11(10.8)$

$27(3.8) \quad 0.0013$

Table 2.6 Factors possible influencing recurrence 


\section{Discussion}

Our data show that high cure rates can be obtained when high risk BCCs are treated by MMS. When reviewing the literature it became clear that although much has been written about MMS in the treatment of BCC (479 articles reporting about MMS and BCC), there are only eight studies that report on valid five-year recurrence rates which is a major criterion to judge the effectiveness of any cancer treatment (table 2.7).97.104.106.107.202-205 In addition, there is a great diversity in study design and different studies are not comparable for several reasons. Some (older) articles report recurrence rates of the fixed tissue technique or combine these with the results of the fresh tissue technique. ${ }^{97,107,202,204}$ Recently published studies report on the fresh tissue technique. ${ }^{104,106,203}$ Furthermore, these studies report about different localisations and size of tumours whenever reported. In all studies recurrence rates after treatment of a primary $\mathrm{BCC}$ are lower than after treatment of a recurrent $\mathrm{BCC}$, around $1 \%$ versus approximately $6-7 \%$. Recurrence rates mentioned by Wennberg et al. are much higher than recurrence rates mentioned in other studies. ${ }^{106}$ They explain their higher recurrence rates by being the only Mohs' centre in a large area, so only very large and aggressive $\mathrm{BCCs}$ were treated. Wennberg et al. is the only group reporting recurrence rates by means of a survival analysis. ${ }^{106}$ The previous mentioned authors use the strict five-year recurrence rates, thereby leaving out the results of the patients who did not complete the five-year follow-up period.

This study is the largest study since the reports of recurrence rates by Mohs, with a high percentage of patients with $\mathrm{BCC}$ who completed a five-year follow-up. Although it is a retrospective study, the strength of this study is that the inclusion criteria for MMS did not change during the study period and a large percentage of patients completed the five-year followup period. As mentioned by reviewers previously, the best way to calculate the recurrence rate is by using survival analysis, because it takes all tumours into account for the time they have been in follow-up, even if there was no completed five-year follow-up. ${ }^{95,125,206}$ Thissen et al. concluded in their systematic review of treatment for primary BCC that because of lack in uniformity in calculating the recurrence rates, different treatment modalities are difficult to compare. ${ }^{95}$ However, they proved that the lowest recurrence rates are provided by MMS. They found recurrence rates for other treatment modalities between $5.3 \%$ after surgical excision and $18.8 \%$ after curettage and electrodessication for primary BCCs. Recurrence rates after treatment of a recurrent BCC lie between $9.8 \%$ after radiotherapy and $40 \%$ after curettage and electrodesiccation. ${ }^{105}$ The best way to compare two treatment modalities is by a randomised controlled trial. From this large study we can conclude that low recurrence rates after treatment of facial BCC by MMS can be obtained.We noticed that $75 \%$ of all recurrences appeared within three years after the operation. This matches with the results of other reviewers of recurrence rates. ${ }^{96.206}$ Recurrences after treatment of a primary BCC appear not significantly sooner than after treatment of a recurrent BCC. However, a large 
percentage of recurrent BCCs still recurred after five years of follow-up. For this reason we recommend a follow-up period of five years for single primary BCCs and a period of seven till ten years after treatment of a recurrent $B C C$. A person with a $B C C$ has a $44 \%$ chance of developing a second $\mathrm{BCC} .{ }^{207}$ Because the highest chance is within the first year, this follow-up period will also lead to early recognition of these BCCs.Most BCCs were eradicated within two stages suggesting that most Mohs' procedures were finished in a few hours. Other authors have shown that costs of a Mohs' procedure are comparable to that of a surgical excision and that cost of a Mohs' procedure will rise with increasing stages. ${ }^{109.208}$ So because $71 \%$ of all BCCs was eradicated within two stages, we conclude that most Mohs' procedures are not that time-consuming and therefore, not that expensive as sometimes is suggested. From the fact that only $24.7 \%$ of all BCCs was eradicated in one stage, one could conclude that only tumours with a strict indication (indistinct tumour borders, aggressive histopathologic subtype and/ or large tumours) were included for treatment by MMS. So when these strict selection criteria for MMS are lived up to, MMS is less expensive than a recurrence treated too late. We found that predictive values for recurrence are an aggressive histopathologic subtype, four or more Mohs' stages and a large defect size $>40$ $\mathrm{cm}$ in diameter). We also found a significant difference between recurrence rates after a primary and a recurrent $\mathrm{BCC}$. These predictive values are also known to be of importance in the treatment of BCC by other treatment modalities. ${ }^{94,105,209,210}$ This study is an extension of research in MMS, first described in $1996 .{ }^{201}$ in that study a five-year recurrence rate of $2 \%$ was reported which is less than the recurrence rates in this study. In that group of patients an additional excision margin of 2-3 $\mathrm{mm}$ was taken after the area was tumour free. So perhaps one of the largest advantages of MMS, namely the tissue sparing aspect, is a disadvantage in these large tumours. However, compared to other treatment modalities, recurrence rates are consistently lower. We found significantly higher recurrence rates in the first two treatment years. This might be explained by the fact that in the first years that MMS was practised at the University Hospital of Maastricht, only very large, aggressive and recurrent tumours were treated. Another explanation might be the fact that the surgeons were less experienced indicating that the learning curve is also an important factor. A Mohs surgeon has to control besides the surgical technique also the graphically mapping and histopathological examination. Other authors have tried to explain why recurrences still can occur after treatment with MMS, where theoretically $100 \%$ of the resection margins is viewed. ${ }^{211-214}$ Eliezri and Dzubow both report cases where the scar tissue (in case of biopsy scar or a recurrent $\mathrm{BCC}$ ) is not completely excised and therefore tumour is missed. ${ }^{212,214}$ These cases stress the importance of complete excision of all scar tissue to ensure that all tumour cells are eradicated and to prevent a recurrence. Hruza analysed their recurrences and found an explanation for recurrence in $77 \%$ of cases. ${ }^{214}$ The most common causes for recurrence were due to incomplete Mohs' slides (lacking part of the epidermis or dermis) so 
resection margins were not completely visible and tumour could be missed. We recently reported that there is a $2 \%$ fault in detecting BCC in Mohs' sections stained with haematoxylin and eosin, especially in morpheic BCC. ${ }^{213}$ Another consideration in analysis of recurrence is the possibility of non-contiguous growth of the $\mathrm{BCC}$. There has been some discussion whether, especially the superficially growing $\mathrm{BCC}$, is a non-contiguously growing tumour. However, a three-dimensional study proved that even a superficial growing BCC is a contiguously growing tumour. ${ }^{215}$ So, in our opinion, if the Mohs' procedure is properly performed and all scar tissue is excised, the reason for local recurrence is a technical failure and therefore complete frozen sections, visibly controlled with colour matching of the edges, are mandatory in MMS. As we mentioned before, besides a technical failure, mistakes in colouring or mapping can, especially during the training period, occur and may be a cause of recurrence.

\begin{tabular}{|c|c|c|c|c|c|c|c|c|c|c|c|c|}
\hline Author & $\mathrm{N}$ & $\begin{array}{l}\text { Prim } \\
\text { (n) }\end{array}$ & $\begin{array}{l}\operatorname{Rec} \\
(n)\end{array}$ & Died & $\begin{array}{l}\text { Lost } \\
\mathrm{FU} /<5 \\
\text { years }\end{array}$ & $\begin{array}{l}\text { Prim } \\
\text { 5-yr FU }\end{array}$ & $\begin{array}{c}\operatorname{Rec} \\
5-y r F U\end{array}$ & $\begin{array}{l}\text { Local- } \\
\text { isation }\end{array}$ & $\begin{array}{l}\text { mean } \\
\text { size } \\
(\mathrm{cm})\end{array}$ & $\begin{array}{c}\text { Rec } \\
\% \\
\text { prim }\end{array}$ & $\begin{array}{l}\text { Rec } \\
\% \text { rec }\end{array}$ & $\begin{array}{l}\text { fixed/ } \\
\text { fresh }\end{array}$ \\
\hline $\begin{array}{l}\text { Tromovitch, } \\
1966^{204}\end{array}$ & 102 & & 102 & & & & 102 & $\begin{array}{l}\text { most } \\
\text { face }\end{array}$ & $0.5-12$ & & 6.9 & fixed \\
\hline $\begin{array}{l}\text { Sakura, } \\
1979^{97}\end{array}$ & 40 & & 40 & & & & 40 & head & 1.9 & & 12 & fixed \\
\hline $\begin{array}{l}\text { Mohs, } \\
1981^{202}\end{array}$ & 576 & - & - & 116 & 16 & 385 & 59 & scalp & - & 0 & 6.8 & $\begin{array}{l}\text { fixed } \\
\text { and } \\
\text { fresh }\end{array}$ \\
\hline $\begin{array}{l}\text { Robins, } \\
1985^{205}\end{array}$ & 631 & - & - & & & 318 & 313 & $\begin{array}{l}\text { peri- } \\
\text { ocular }\end{array}$ & - & 1.9 & 6.4 & - \\
\hline $\begin{array}{l}\text { Mohs, } \\
1986^{203}\end{array}$ & 1773 & - & - & 285 & 74 & 1124 & 290 & eye & - & 0.6 & 7.6 & fresh \\
\hline $\begin{array}{l}\text { Mohs, } \\
1988^{107}\end{array}$ & 1213 & - & - & 240 & 44 & 748 & 181 & ear & - & 1.7 & 7.8 & $\begin{array}{l}\text { fixed } \\
\text { and } \\
\text { fresh }\end{array}$ \\
\hline $\begin{array}{l}\text { Julian, } \\
1997^{104}\end{array}$ & 228 & - & - & 19 & 78 & 58 & 83 & - & 1.9 & 1.7 & 4.8 & fresh \\
\hline $\begin{array}{l}\text { Wennberg, } \\
1999^{106} \\
\text { - = not menti }\end{array}$ & $\begin{array}{l}248 \\
\text { oned }\end{array}$ & - & - & & 20 & 87 & 141 & $\begin{array}{c}\text { most } \\
\text { head/ } \\
\text { neck }\end{array}$ & - & 6.5 & 10 & fresh \\
\hline
\end{tabular}

Table 2.7 Studies reporting on five-year recurrence rate for primary and/ or recurrent BCC

\section{Conclusion}

Our data show that very low recurrence rates in treatment of facial BCC can be obtained even in a selected group of large and aggressive BCCs. Comparing our results with literature results MMS consistently continues to provide the lowest recurrence rates. It therefore is the treatment of first choice for primary facial BCCs of an aggressive histopathologic subtype and recurrent BCCs of the face. 


\section{3 \\ Imiquimod 5\% cream and photodynamic therapy as adjuvant treatment after incomplete Mohs' micrographic surgery for large, mixed-type basal cell carcinoma}

Background: Surgical excision, including Mohs' micrographic surgery (MMS), is the treatment of first choice for basal cell carcinoma. Occasionally, the Mohs' procedure has to be discontinued before completion for unforeseen reasons.

Objective: Possibilities were sought for the adjuvant treatment of patients in which superficial basal cell carcinoma) remained after incomplete MMS.

Methods and Materials: We report seven patients in which MMS for their large BCCs was ceased for different unforeseen reasons, with remaining superficial fields of $B C C$ after the aggressive and deeply localised part had been removed. Three weeks after closure of the defect, adjuvant therapy for the superficial remnants of the tumour was given either with imiquimod $5 \%$ cream or with photodynamic therapy (PDT).

Results: The treatments were accepted well with no serious side effects and good cosmetic results. No recurrences were seen after a follow-up period of 13 to 30 months.

Conclusion: A combination of surgical intervention for the central deeper part of the BCC and topical imiquimod $5 \%$ cream or PDT for the superficial part at the border is a therapeutic option for those patients with multiple facial BCCs, severe sun damage and extensive surgery in the past, for whom completion of the Mohs' surgical procedure for the entire tumour is not possible for various reasons. 


\section{Introduction}

Basal cell carcinoma (BCC) is the most common non-melanoma skin cancer (NMSC) worldwide. Incidence rates are still increasing with more and more patients developing multiple and large facial BCCs at younger age. ${ }^{4}$ Mohs' micrographic surgery (MMS) has proven to be a highly effective treatment for many skin cancers and especially NMSC. ${ }^{95}$ MMS is preferred for aggressive, recurrent and large BCCs especially in the face. ${ }^{196}$ However, in cases with multiple facial BCCs surgery can not be performed endlessly without a serious risk of loss of functionality or cosmetic result. Occasionally, the Mohs' procedure has to be discontinued before completion for unforeseen reasons, such as ongoing positive skin margins, bone invasion or inability of the patient to tolerate further surgery. Adjuvant treatments for the remaining tumour such as a second surgical procedure, radiotherapy, dermabrasion or observation have been described. ${ }^{105}$

Topical imiquimod $5 \%$ cream stimulates the induction of monocyte-macrophage derived cytokines such as interferon, which results in a Th1-mediated cellular antiviral and antitumoural immune response and thereby enhances the removal of neoplastic cells. ${ }^{217,218}$ Clinical studies have shown that topical imiquimod $5 \%$ cream is particularly efficacious in clearing the superficial growing subtype of $\mathrm{BCC}$, with complete response rates between 50 and $100 \%{ }^{74,167.170,172}$

There are several histopathological subtypes of $B C C$, which can be subdivided into aggressive (morphea, micronodular and infiltrative types) and non-aggressive (nodular and superficial types) tumours. ${ }^{219}$ Superficial BCC (SBCC) is characterised histologically by small buds of basaloid cells in continuity with the epidermis, protruding into the upper dermis. In the past, this type has been mentioned in literature as multicentric or multifocal superficial BCC. Computerised reconstruction demonstrated anastomosing and interconnecting complexes between the tumour buds, which proves that the names multicentric and multifocal are technically incorrect. ${ }^{215}$ The tumour type will often be determined on the basis of a biopsy. However, this biopsy may be an unrepresentative sample because many BCCs consist of mixed subtypes, combining a nodular or aggressive type in the centre with the superficial type at the borders. ${ }^{220}$ This phenomenon is seen particularly in patients with multiple facial skin cancers and severe sun damage. Surgical excision of all these superficial fields might result in extremely large defects.

Reports are given of seven patients with large facial BCCs that were treated with MMS in which the surgical procedure was discontinued for different unforeseen reasons causing reconsideration as to whether or not to continue the initial treatment.

In three cases, the defects were closed and two weeks after removal of the stitches adjuvant therapy for the superficial remnants of the tumour with imiquimod $5 \%$ cream was started.

In four cases the surroundings of the surgical defect were treated with PDT by using a light 
source of $600-730 \mathrm{~nm}$ (Waldmann PDT 1200 unit), with fluence rates ranging from 90 to $100 \mathrm{~mW} / \mathrm{cm}^{2}$ and light doses from 60 to $100 \mathrm{~J} / \mathrm{cm}^{2}$, after a four-hour application of freshly prepared $20 \% 5$-aminolevulinic acid oil in water.

\section{Patient 1}

A 77 year-old Caucasian male with a history of excessive sun exposure had a proven recurrent, infiltrating nodular $\mathrm{BCC}(13 \times 18 \mathrm{~mm})$ in the right temporal area. The primary tumour had been treated with radiotherapy eight years ago. The medical history revealed multiple facial BCCs of different histological subtypes, one squamous cell carcinoma $\left(T_{1} N_{0} M_{0}\right)$ and solar keratosis all treated by surgical excision and/or radiotherapy or cryotherapy.

The tumour was treated by MMS. After four stages and 24 frozen sections the bulk of the tumour had been removed, but there was still superficial growing BCC in several sections. (Fig.2.9a/b) MMS was terminated then because of the poor clinical demarcation, the size $(54 \times 78 \mathrm{~mm})$ and localisation of the defect in an area treated by surgery and radiotherapy several times in the past. The defect was closed with a rotation flap from the pre-auricular area. The whole scar including a surrounding margin of at least $1 \mathrm{~cm}$ was treated with topical imiquimod $5 \%$ cream three days per week under occlusion for six weeks in order to treat the remaining superficial BCC. Mild erythema and crusts were observed during the second half of the treatment period. Severe adverse events did not occur. After 30 months' follow-up there was still no clinical sign of tumour recurrence.

\section{Patient 2}

A 58 year-old Caucasian woman was sent for MMS because of an incompletely excised morpheic BCC in the left pre-auricular area. Only the scar $(5 \times 30 \mathrm{~mm})$ of the previous excision was visible; tumour could not be detected clinically. After four stages and eighteen frozen sections, there was still superficial $B C C$ in several sections. The surgical procedure was discontinued because of severe backache such that the patient could not lay down any longer. The defect $(18 \times 67 \mathrm{~mm})$ was closed by primary approximation.

Subsequently, the scar and a margin of approximately $1 \mathrm{~cm}$ were treated with topical imiquimod $5 \%$ cream five days per week for six weeks. There were no adverse events other than mild erythema. There was no clinical sign of tumour recurrence during 24 months' follow-up.

\section{Patient 3}

A62 year-old Caucasian man suffering from pulmonary and cardiac disease, had an easily bleeding, recurrent nodular BCC $(18 \times 23 \mathrm{~mm})$ after cryosurgery on the right cheek. His skin revealed typical signs of severe sun damage and he had been treated for multiple BCCs in the head and neck area in the past (surgical excision / cryosurgery). The present tumour was treated with MMS because of the recurrent character and size. After three stages and 
fifteen frozen sections, $S B C C$ was still present in three sections. The Mohs' procedure had to be terminated at that moment due to severe dyspnoea of the patient. The defect of $25 \times 46 \mathrm{~mm}$ was closed by primary approximation.

Imiquimod $5 \%$ cream was applied five days per week for six weeks on and approximately $1 \mathrm{~cm}$ around the scar. After four weeks treatment was interrupted for one week due to short hospitalisation for an unrelated reason. Sixteen months post treatment there was no clinical sign of tumour recurrence.

\section{Patient 4}

A 7 five-year-old male patient with a history of severe sun exposure had a primary tumour of $10 \times 7 \mathrm{~mm}$, located on his glabella. Histopathologic examination performed by punch biopsy showed an infiltrative growing BCC. His medical history revealed Merkel cell carcinoma cranial to his right eye that had been excised in 1997. He had also been treated for multiple actinic keratoses, two prior BCCs on his cheek and forehead and a large, deep infiltrating squamous cell carcinoma $\left(S C C, T_{1} N_{0} M_{0}\right)$ in the left medial canthal region, for which an excenteration of the bulbus oculi and a neck dissection was performed in 2000 . The BCC on his glabella was treated with MMS. After two stages and eight frozen sections, sBCC was seen still to be present on one of the slides corresponding with the right side of the nose. The Mohs' procedure was then terminated, because he had little healthy skin left for reconstruction due to extensive actinic damage in the face. The defect of $15 \times 10 \mathrm{~mm}$ was closed by primary approximation. The residual SBCC was treated after five weeks with PDT $\left(100 \mathrm{~mW} / \mathrm{cm}^{2}, 100 \mathrm{~J} / \mathrm{cm}^{2}\right)$. Sixteen months post treatment there was no clinical sign of a recurrent tumour.

\section{Patient 5}

An otherwise healthy fiftyfive-year old male was seen with a recurrent tumour in the right temporal zone six months after MMS for a mixed nodular, superficial and infiltrative subtype BCC. Histopathologic examination showed a superficial BCC. He had been treated before for multiple BCCs of different subtypes in his face with MMS and surgical excision. This tumour was again treated by MMS because of the recurrent character and after one stage and four frozen sections there was superficial growing BCC apparent in two of four slides. Three punch biopsies taken within the surrounding area of several centimetres, in which tumour tissue was difficult to delineate, also showed sBCC. The Mohs' procedure was terminated because of the superficial aspect of the tumour and the wide area in which the sBCC extended. The size of the defect was $23 \times 18 \mathrm{~mm}$ and this was closed by primary approximation. A five-centimetre surrounding area was treated with PDT after ten weeks as described before. The following settings were used: $90 \mathrm{~mW} / \mathrm{cm}^{2}, 100 \mathrm{~J} / \mathrm{cm}^{2}$ ). After 26 months there was no clinical sign of a recurrence. 


\section{Patient 6}

An 80 years-old, pulmonary and cardiac-compromised male patient with a history of extensive sun exposure was sent to the clinic with a recurrent $B C C$ on the right side of his forehead. The primary tumour had been treated with surgical excision several years before. He had been treated for multiple BCCs in the head and neck region in the previous years by surgical excision and cryotherapy. Histopathologic examination of a punch biopsy showed a nodular subtype BCC.

He was treated with MMS because of the recurrent character of the BCC. Two stages and ten frozen sections later, there was $\mathrm{SBCC}$ remaining in one slide. At this time, the defect measured $40 \times 50 \mathrm{~mm}$. The procedure was terminated because of severe dyspnoea of the patient, the defect was partially closed by a purr string and the remaining defect was left open for secondary intention healing. Eight weeks later, after healing of the wound, PDT was performed as described before $\left(100 \mathrm{~mW} / \mathrm{cm}^{2}, 60 \mathrm{~J} / \mathrm{cm}^{2}\right)$ with a margin of approximately $2 \mathrm{~cm}$. During the follow-up period five new BCCs have been treated, one of which was located on his forehead, but outside the area treated by PDT. No recurrences appeared within the follow-up period of 27 months.

\section{Patient 7}

An 82 year-old man has been treated at the clinic for five SCCs and eleven BCCs of different subtypes in the head and neck region, with surgical excision, MMS and cryosurgery. At a regular oncology check-up two new primary tumours of $21 \times 18 \mathrm{~mm}$ and $8 \mathrm{~mm}$ were noticed in the pre-auricular zone on the left side (Figure 2.10a). Histopathological examination after biopsy of the largest tumour showed a solid growing BCC. The patient was treated with MMS because of the localisation and size of the tumour. After five stages and fourteen frozen sections there was SBCC apparent in three slides (Figures 2.11a/b). The procedure was terminated when a defect of $66 \times 39 \mathrm{~mm}$ remained (Figure 2.10b). The defect was partially closed by primary approximation and the remainder with a full thickness skin graft from his supra-clavicular area. Again, PDT was performed as described before with a $2 \mathrm{~cm}$ margin. Thirteen months later there were still no signs of recurrence of the tumour (Figure 2.10c). Several months after this last physical examination, the patient died of an unrelated cause.

\section{Discussion}

There are many different treatment modalities for BCCs, including surgical excision, MMS, cryosurgery, radiotherapy, and PDT topical immunotherapy. ${ }^{196}$ MMS provides both low recurrence rates and maximal preservation of healthy tissue around the tumour, which is necessary to close the defect and obtain cosmetically and functionally good results. ${ }^{104-106}$ Preservation of healthy tissue is particularly important in patients with multiple skin can- 
cers who are at risk of developing new NMSC in the future, for instance patients with basal cell nevus syndrome, patients with immunosuppressive therapy and patients with a history of extensive sun exposure.

In patients with extensive sun damage and multiple tumours, BCCs often consist of mixed histological subtypes showing the nodular or more aggressive type in the centre and depth and superficial type at the borders. Clearing of these superficial fields with MMS requires several extra stages leading to larger defects and in these cases perhaps to unwanted loss of tissue. Otherwise MMS is a time-consuming intervention and especially for older or physically disabled patients the procedure may become exhausting when multiple stages are necessary.

After the aggressive and deeper localised part of the BCC has been removed by MMS, the remaining superficial fields may be treated effectively by non-invasive therapies. In the cases described here, no recurrences were seen after a follow-up period of 13 to 30 months. The risk of recurrence and the management after incomplete treatment has often been debated. ${ }^{221}$ Recurrence rates in the case of positive margins as quoted in the literature vary from 26 to $100 \% .^{222-224}$ However, as recurrent BCCs are more difficult to treat, we aim at complete eradication of the tumour, consistent with advice found in the literature. ${ }^{222}$

\section{Conclusion}

The patients described here are good examples of those encountered by dermatologists and illustrate the dilemmas that can occur during a Mohs' procedure as to whether or not to continue in cases where there is incompleteness of the sections, as demonstrated in the frozen sections due to superficial tumour tissue which sometimes extends far into the surrounding areas.

These series of cases suggest a potential role for imiquimod and PDT as adjuvant therapy for remnants of superficial BCC after multiple stages of MMS for mixed-type BCC in patients with extensive sun damage, multiple facial BCCs and extensive surgery in the past, or for those cases in which MMS has to be terminated before completion for unforeseen reasons. Accurate follow-up studies are necessary to determine the long-term efficacy of imiquimod $5 \%$ and topical PDT as part of a combination of treatments. 
Figure 2.10 Patient 7

a. Tumour before treatment

b. Final defect after surgery

c. Treated area 13 months after PDT
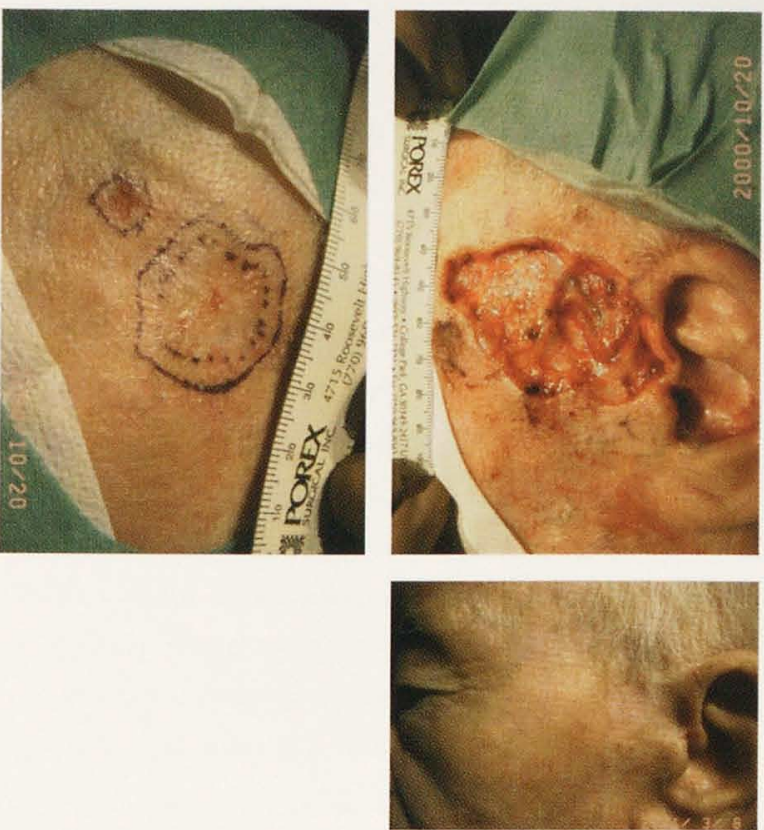

Figure 2.9 Histological aspect of the tumour of patient 1

2.9 a. Histopathological aspect of the BCC in the first slides

$2.9 \mathrm{~b}$. Histopathological appearance of the same BCC with a small superficial tumour bud at time of termination of the Mohs' procedure
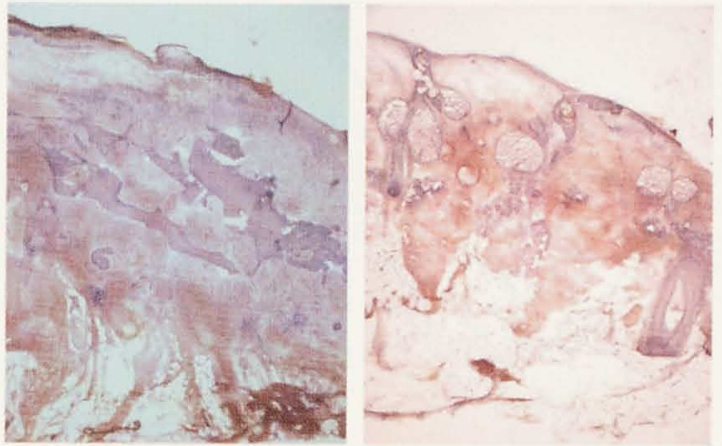

Figure 2.11 Histological aspect of the tumour of patient 7

2.11 a. (Micro-) nodular BCC in early Mohs' stage

2.11 b. Superficial BCC

in latter Mohs' stage.
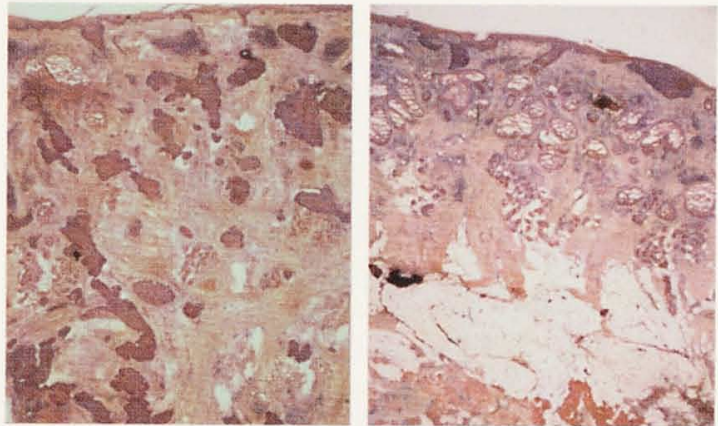


\section{4 \\ Does systemic azithromycin improve the survival of a full thickness graft on the nose?}

Background: The healing of full thickness grafts on the nose does not always proceed without problems. Partial or entire necrosis of the graft is likely to lead to less favourable cosmetic results and prolonged wound care. No consensus exists as to the use of systemic antibiotics to increase the success rate of survival of a full thickness skin graft on the nose after non-melanoma skin cancer surgery.

Objective: The objective of the study was to evaluate the effect of systemic azithromycin on the survival of full thickness grafts on the nose.

Methods: A randomised controlled trial was performed in which the use of azithromycin was compared to no antibiotic treatment in 30 patients who underwent a full thickness graft reconstruction of a surgical defect on the nose after surgery of non-melanoma skin cancer. The percentage survival of the graft was the main outcome measure.

Results: A statistically significant difference in favour of the grafts treated with systemic azithromycin was seen $(p=0.002)$. Of all the variables analysed, only smoking had a significant negative effect on the survival of the skin graft.

Conclusions: Systemic antibiotics with accurate bacterial spectrum such as azithromycin may be advised in full thickness skin graft reconstruction after surgery of non-melanoma skin cancer of the nose. Smoking should be strongly discouraged. 


\section{Introduction}

Surgical defects after skin cancer surgery can be reconstructed in many different ways. The simplest and often the best option is primary closure. If this is not possible due to size or localisation of the defect, a transposition, advancement or rotation flap should be considered. Although cosmetically not always the best option due to skin colour and texture differences, a full thickness graft (FTG) can be a good alternative and is often applied.

Personal experiences within the authors' department are that FTGs on the nose more often lead to necrosis than FTGs elsewhere in the face. A possible explanation for this may be that bacteria in or on the nose, more often lead to a sub-clinical wound infection, or that the circulation is compromised. Success rates of full thickness grafts as mentioned in the literature vary and are difficult to compare due to differences in method and time of evaluation, as well as donor and recipient site characteristics. Robinson and Dillig found that $30 \%$ of approximately 200 nasal FTG treated with topical antibacterial ointment (in a group of non-smokers) showed partial graft loss, mostly confined to less than $25 \%$ surface. ${ }^{231}$ Other authors report of $14 \%$ of the grafts (of which $73 \%$ nasal) showing some degree of necrosis. ${ }^{232}$ Partial or entire necrosis of the graft is likely to lead to less favourable cosmetic results and more problems for the patients, such as prolonged wound care.

No consensus as to the use of systemic antibiotics to increase the success rate of survival of an FTG. To prevent the risk of resistance by unnecessary use of antibiotics, the ratio for the use of the antibiotics should be investigated and proven.

This study was started to evaluate the effect of azithromycin, a broad-spectrum, systemic antibiotic, on the survival percentage of an FTG on the nose. The study also investigated several tumour-, treatment- and patient-related variables, which could influence the survival of the FTG.

\section{Methods}

The study was designed as a randomised controlled trial (RCT). Patients with non-melanoma skin cancer (NMSC) on the nose, who were treated with Mohs' micrographic surgery (MMS) or surgical excision (SE), and for whom the dermatological surgeon decided for reconstruction by FTG, were eligible for randomisation. Randomisation was performed centrally by telephone through a person who was not involved in the trial. This person used a computer-generated random allocation scheme. A total of 30 patients with 30 defects were included. Sixteen patients received antibiotic therapy (group 1) and fourteen received no post-operative antibiotics (group 2). The patients within group 1 received one oral dose of $500 \mathrm{mg}$ azithromycin for three days. Cultures were taken from the nasal cavity as well as the defect before reconstruction. Reconstruction took place immediately after surgery during the same session. Sterile petrolatum dressings were placed on the FTG in all cases, and a standardised pressure bandage or a bandage without pressure was placed on top 
of this. Patient-related information of interest was scored, such as smoking behaviour, concomitant diseases, medication, sun exposure, sex and age. The following tumourand surgery-related variables were scored: localisation, histological diagnosis, defect size $(\mathrm{mm})$, duration of the total procedure, localisation of the donor site, type of bandage used after surgery and outcome of the bacterial cultures.

The patients returned for wound control after five-seven days and ten-fourteen days. During these visits the survival of the FTG was scored as a percentage of the surface, by assessment of the colour, texture, induration, swelling and exudation of the graft and surrounding skin. A physician who was not involved in the trial, but who was not blinded to the treatment groups, performed this assessment. Additional cultures were taken when the FTG appeared to be infected.

\section{Statistical analysis}

Data was analysed using SPSS-PC for Windows. The Independent-Samples T-test was used to determine the difference between both groups. The possible influence on the survival of the FTG was analysed for several determinants using the Independent-Samples T-test and One-Way ANOVA. A p-value of 0.05 or less was considered to indicate statistical significance.

\section{Results}

The mean age of the patients was 69 years (range $48-88$ years). There were more men than women (60\% vs. $40 \%$ ). Five patients were known to smoke (16.7\%), whereas 24 patients were non-smokers ( $80 \%)$. This information was lacking for one patient. Hypertension was a frequent concomitant condition, as were cardiac disease and TIA, related to the relatively high age of the patient population. These conditions were equally distributed over the two groups. Most patients used medication, which was scored and will be discussed later.

Almost all tumours were basal cell carcinomas ( $n=28,93.3 \%$ ); the remaining two concerned squamous cell carcinomas. The mean size of the tumours was $11.2 \mathrm{~mm}$ (range $4-28 \mathrm{~mm})$. Fourteen tumours $(46.7 \%)$ were located on the nasal ala, nine $(30.0 \%)$ on the tip of the nose and seven (23.3\%) on the dorsum of the nose. The mean defect size was $17.5 \mathrm{~mm}$ (range 10-31). The most common donor site was the pre-auricular area $(n=26$, $86.7 \%)$. Other donor sites were retro-auricular $(n=2,6.7 \%)$, medial site upper arm $(n=1$, $3.3 \%)$ and the glabella region $(n=1,3.3 \%)$.

The bacterial cultures of the wound bed showed no growth at all in nineteen cases, commensally bacteria (unspecified) were cultured twice and Proteus mirabilis once. The cultures of the nasal cavity showed no growth at all in nine cases, and commensally bacteria were cultured eleven times. Four patients turned out to be carrier of Staphylococcus 
aureus (S.aureus) intranasal. S.aureus was also found in the wound bed in two of these patients. Three patients showed growth of other specified bacteria in the nose, Moraxella catarrhalis twice and Proteus mirabilis once. Cultures of the nose were not taken three times and from the wound bed six times.

The average duration of the procedure was 150 minutes with a wide range (45-330 min.) due to the difference in surgical procedure (SE or MMS).

A pressure bandage was applied twenty-six times. A bandage without pressure was applied in the remaining three cases. This information was lacking in one case, .

Patient and tumour characteristics per treatment group are shown in Table 2.8. Except for the tumour localisation, all characteristics were distributed equally over both groups. Relatively more tumours were localised on the nasal ala (10 vs. 4) in group 1 whereas more tumours were localised on the back of the nose (5 vs. 2) in group 2.

The mean percentage survival of the grafts after seven days was $83.0 \%$ within group 1 versus $63.8 \%$ of the patients within group $2(p=0.197)$ (Table 2.9). The mean percentage survival of the grafts from group 1 after ten days was $86.6 \%$ versus $36.2 \%$ of the patients within group 2 . This difference is statistically significant $(p=0.002)$. The distribution of survival percentages per treatment group is illustrated in Figures 2.12a-b.

None of the 30 FTGs showed clear signs of infection, such as erythema, induration or pus at any time, therefore no adjuvant cultures were taken during the control visits.

The variables smoking, defect size, duration of the procedure, type of dressing, localisation and bacterial load were analysed to find possible effects on the survival of the FTG (Table 2.10).

The group of smokers $(n=5)$, showed a lower survival percentage of the graft compared to the non-smokers after ten days $(2.0 \%$ vs. $75.9 \%, p<0.001$.) The defect size was dichotomised into defect sizes $2.0 \mathrm{~cm}^{2}$ or smaller and defects larger than $2.0 \mathrm{~cm}^{2}$. A slightly higher survival percentage in the group with the large defects was found at the first followup visit. This difference increased to $56.3 \%$ vs. $72.1 \%$ in favour of the patients with large defects after ten-fourteen days $(p=0.326)$.

A shorter duration of the procedure led to a slightly better survival of the FTG. However, this difference was not significant after ten days $(72.7 \%$ vs. $56.9 \%, p=0.332)$.

The four nasal carriers of S.aureus had no significant worse outcome and a slightly lower survival percentage after five-seven that was no longer apparent after ten-fourteen days $(p=0.541)$.

Localisation and the type of dressing did not influence the graft survival significantly $(p=0.746$ and $p=0.176$, respectively). 


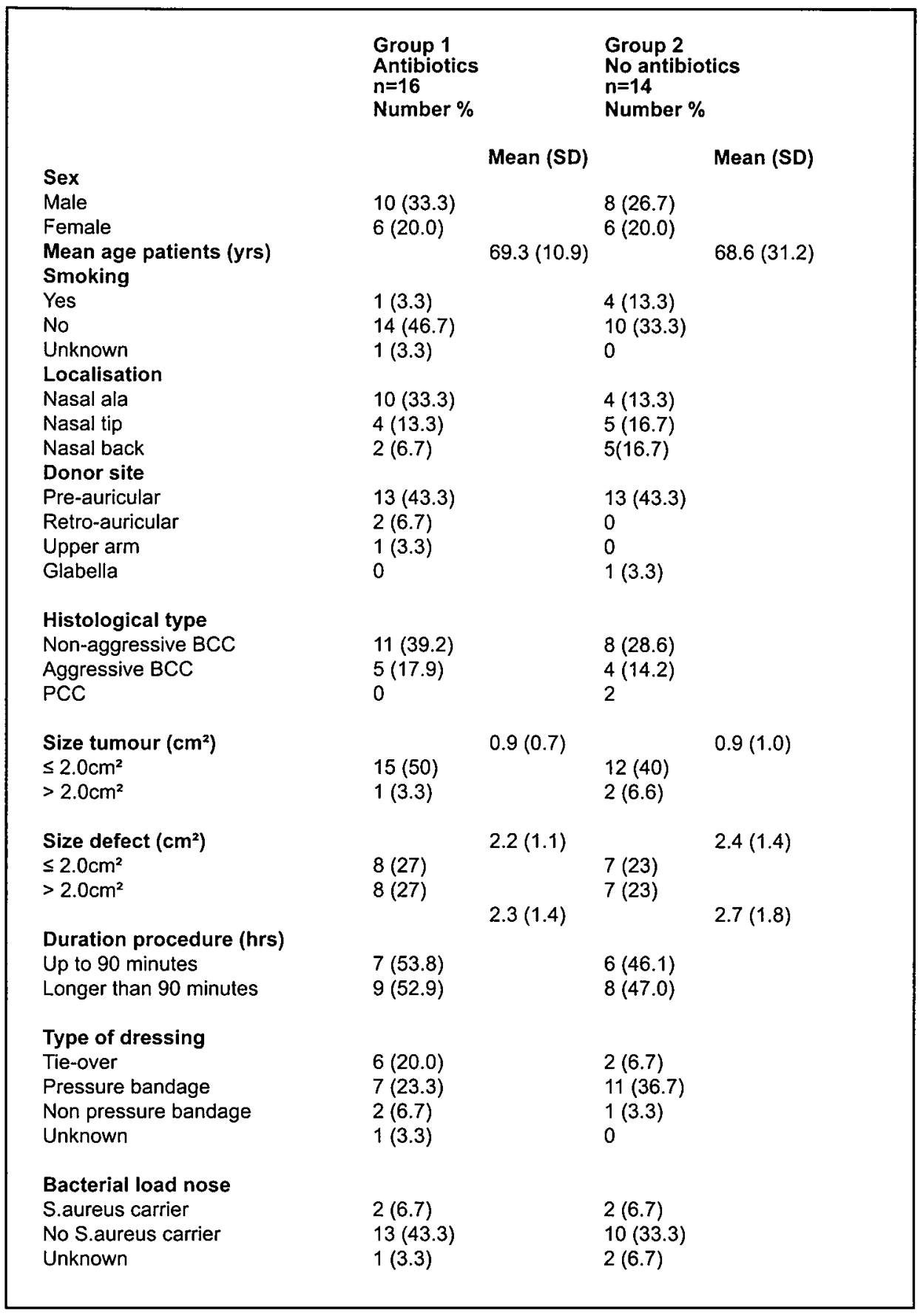

Table 2.8 Tumour- and patients characteristics 
Group $1 \quad$ Group $2 \quad$ Independent

Antibiotics No antibiotics Samples T-test, $n=16$ $n=14$

Number \% Number \% p-value

Survival FTG day 7

Mean survival (\%) (SD)

$83.0(0-100) \quad 63.8(0-100) \quad 0.197$

Survival FTG day 10

Mean survival (\%) (range)

$86.6(0-100)$

$36.2(0-100)$

0.002

Table 2.9 Survival of FTG

\begin{tabular}{|c|c|c|}
\hline & $\begin{array}{l}\text { Mean Survival } \\
\text { FTG }(\%) \\
\text { day } 5-7\end{array}$ & $\begin{array}{l}\text { Mean Survival } \\
\text { FTG }(\%) \\
\text { day } 10-14\end{array}$ \\
\hline \multicolumn{3}{|l|}{ Defect size } \\
\hline$\leq 2.0 \mathrm{~cm}^{2}$ & 71.2 & 56.3 \\
\hline$>2.0 \mathrm{~cm}^{2}$ & 76.7 & 72.1 \\
\hline$p$-value & 0.708 & 0.326 \\
\hline \multicolumn{3}{|l|}{ Smoking } \\
\hline yes & 38.0 & 2.0 \\
\hline no & 81.1 & 75.9 \\
\hline p-value & 0.019 & $<0.001$ \\
\hline \multicolumn{3}{|l|}{ Localisation } \\
\hline Ala & 60.0 & 57.9 \\
\hline Tip & 89.3 & 67.2 \\
\hline Back & 82.9 & 73.3 \\
\hline$p$-value & 0.176 & 0.746 \\
\hline \multicolumn{3}{|l|}{ Duration procedure } \\
\hline$\leq 90$ minutes & 82.9 & 72.7 \\
\hline$>90$ minutes & 67.5 & 56.9 \\
\hline$p$-value & 0.293 & 0.332 \\
\hline \multicolumn{3}{|l|}{ Type of dressing } \\
\hline Tie-over & 98.3 & 82.5 \\
\hline Pressure bandage & 68.6 & 53.8 \\
\hline Non pressure bandage & 63.3 & 93.3 \\
\hline$p$-value & 0.230 & 0.137 \\
\hline \multicolumn{3}{|l|}{ S.aureus carrier } \\
\hline No S.aureus & 81.7 & 67.6 \\
\hline S.aureus & 65.5 & 66.7 \\
\hline$p$-value & 0.998 & 0.541 \\
\hline
\end{tabular}

Table 2.10 Determinants of survival of the FTG 


\section{Discussion}

Although the results are based on a small number of patients, the nasal FTGs in this study showed a better survival rate when treated directly post-operatively with azithromycin.

Guidelines have recently been proposed for the use of antibiotics as prophylaxis for surgical site infection in dermatologic surgery, in which tumour-, procedure- and patient-related risk factors should be taken into account. ${ }^{233}$ According to the authors, prophylactic antibiotics are advised for surgical wounds involving inflamed or infected skin, and in patients with a higher risk, such as diabetes mellitus, obesity, chronic renal insufficiency, malnutrition, immunodeficiency or the use of corticosteroids, tobacco and alcohol. Other risk factors for impaired wound healing are non-sterile nature of the procedure, excessive injury to the tissue, haematoma formation, involvement of mucosa, high-tension closures and flaps and grafts with poor blood supply. ${ }^{233}$

The applied type of dressing was not found to significantly influence the survival in this analysis, but the small number of patients means that the analysis must be interpreted with caution. It is well understandable that pressure can prevent haematoma formation, but too tight a dressing can impair the blood circulation and lead to necrosis.

Medications used that might influence the healing process were colchicines, acenocoumarol (group 1), low dose prednisone, tacrolimus and meloxicam (group 2) and acetylsalicylic acid and beta-blocking agents in multiple patients. The use of these potentially relevant medications was distributed evenly among both groups and the number of patients was too small to analyse their influence statistically.

Because Mohs' surgery may extend for many hours, a higher risk for bacterial contamination and wound infection can be expected. These results show that a longer duration of the procedure does not lead to a significant worse survival of the FTG. As proposed in the literature, Mohs' surgical procedures with primary repairs do not necessarily need prophylaxis, but in case of skin flaps and graft reconstruction on the nose and ears, antibiotic prophylaxis can be considered. ${ }^{233}$

To achieve the maximum effect of the prophylaxis, according to the literature, the use of antibiotics must preferably be started in advance, although the results here showed that starting antibiotics directly post-operative is also adequate. ${ }^{233,234}$ An appropriate selection of antibiotic coverage is important. Since dermatologic surgery mostly involves glabrous skin, antibiotics should be aimed at the eradication of Staphylococcus aureus and Streptococcus pyogenes. S.aureus is known to colonise nasal surfaces, making it the most common pathogen in postoperative surgical site infections. The risk of infection is up to nine times higher in carriers of S.aureus. ${ }^{235}$

Azithromycin is an antibiotic agent from the group of macrolids, which is chemically stable and is generally well tolerated. The antibacterial spectrum of azithromycin is effective against Gram-positive organisms and against skin infections, where it has shown to eradicate Sta- 
phylococcus aureus and Streptococcus pyogenes. ${ }^{236}{ }^{237}$ Additionally, the pharmacokinetics allow short dosing schemes because of prolonged levels in the tissue, which is likely to increase patient compliance. ${ }^{238,239}$ Dosing schemes of azithromycin once daily for three days have proven to be highly effective in skin infections. ${ }^{240}$ Theoretically, usage of topical or intraincisional antibiotics can also be considered in the use of infection prevention. ${ }^{241}$

As expected, the difference in survival of the graft between the groups became greater during the follow-up visits. After five to seven days, grafts are often pale or violaceous and critically perfused. It becomes clear whether the graft will survive or not during the following days when the blood supply is restored. The patients receiving antibiotics showed a slight increase in percentage survival over time while the patients without antibiotics showed a marked decrease in survival of their grafts. This can be explained by the effect of the antibiotics in preventing sub-clinical infections. Other qualities of the macrolid should also be considered in explaining the favourable outcome. Animal studies have found clarithromycin to suppress systemic and local inflammatory responses after surgery. ${ }^{236}$ Good efficacy of azithromycin in acne and rosacea may suggest an anti-inflammatory effect besides the antibacterial working mechanism.

There seemed to be a striking negative effect of smoking on the survival of the graft in the study population. This is well explainable due to the effects of smoking. Nicotine impairs healing through vasoconstriction, leading to a reduction of the blood flow and there is also a higher risk of ischemia due to increased platelet adhesion. In addition, proliferation and migration of blood cells and macrophages is reduced by nicotine, as is the migration of fibroblasts, that are essential for wound healing. ${ }^{242,243}$ Smokers are found to have a higher risk of wound infection, which seems to drop again when the smoking is stopped for several weeks prior to surgery. ${ }^{244}$ Although the number of smokers was small, they had a mean survival of their graft during the second visit of $2.0 \%$ compared to $75.9 \%$ in the non-smoking group. Unfortunately, four out of five smokers were in group 2. Two possible explanations for this outcome can be given: either the negative effect of smoking on the graft has led to a lower outcome in the non- antibiotics group, or the lack of antibacterial effect in group 2 has led to a worse outcome in the smokers group. When the smokers are excluded from the statistical analysis, the difference in survival of the graft remains significant between the two groups $(p=0.025)$. This finding on the negative effect of smoking on full thickness grafts is consistent with earlier reports. ${ }^{245,246}$ Patients should be strongly advised to abstain from smoking, preferably as soon as possible before surgery takes place.

It is presumed that necrosis of the FTG leads to a poorer cosmetic results. A paper has recently been published concerning the cosmetic results of nasal FTGs, where all donor sites in the face led to an excellent cosmetic result. No difference was found by the authors regarding defect size and nasal location. ${ }^{247}$ 


\section{Conclusion}

The results from the present study show that the use of systemic azithromycin in FTGs on the nose after cutaneous surgery for NMSC leads to better survival of the grafts. Further studies with larger numbers are desirable to confirm these findings. Better survival of the graft is likely to lead to better cosmetic results, a lower risk of additional surgery and a lower burden to the patient. Therefore, systemic antibiotics with accurate bacterial spectrum such as azithromycin may be advised in this type of surgery. When reconstruction by means of FTG is planned beforehand, patients can be instructed to start with their prescribed medication the day before surgery. Smoking appeared to result in less survival of the graft, and should, therefore, be strongly discouraged.

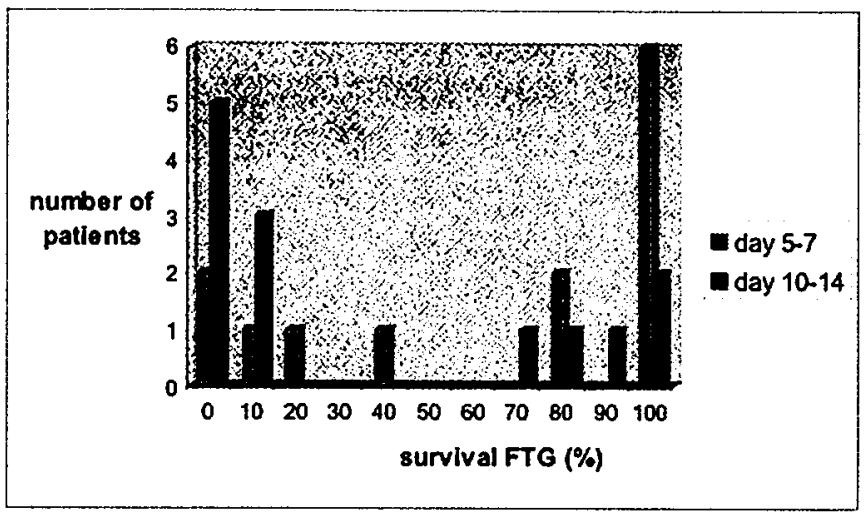

Figure 2.12a Survival of the FTG within group 1 in percentages

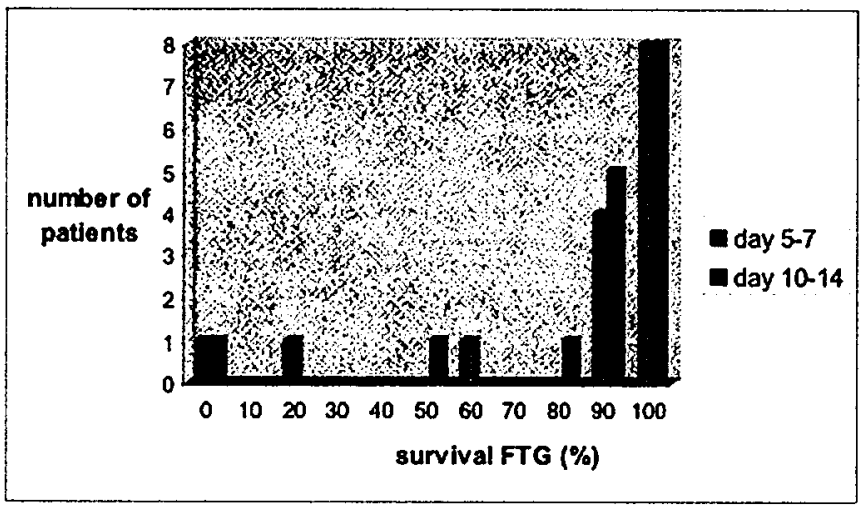

Figure 2.12b Survival of the FTG within group 2 in percentages 



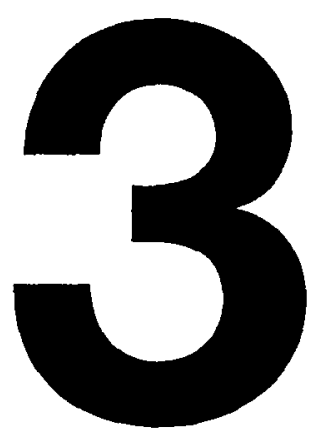

\section{Photodynamic therapy enlightened}

\section{Based on the following publications}

Kuijpers DIM, Mosterd K, Broekhof KGME, Thissen MRTM, Neumann HAM.

Comparison of fractionated photodynamic therapy with surgical excision in the treatment of primary nodular basal cell carcinoma; preliminary results of a randomised controlled trial. Manuscript in preparation

Oosten van EJ, Kuijpers DIM, Thissen MRTM. Different pain sensations in photodynamic therapy of nodular basal cell carcinoma. Results from a prospective trial and a review of the literature. Photodiagnosis and Photodynamic Therapy 2006;3:61-68.

Kuijpers DIM, Thissen MRTM, Thissen CA, Neumann HAM. Similar effectiveness of methyl aminolevulinate and 5-aminolevulinate in topical photodynamic therapy for nodular basal cell carcinoma. J Drugs in Dermatol 2006;5:642-5 


\section{1}

\section{Comparison of fractionated photodynamic therapy with surgical excision in the treatment of primary nodular basal cell carcinoma; preliminary results of a randomised controlled trial}

Background: Fractionated photodynamic therapy (PDT) has proven to be an effective treatment for superficial basal cell carcinoma ( $\mathrm{SBCC}$ ). Its long-term efficacy in nodular basal cell carcinoma ( $\mathrm{BBCC}$ ) has not yet been established.

Objective: The primary objective of the study was to compare the efficacy of PDT and surgical excision (SE) in terms of disease-free rates after five years of follow-up. An interim analysis evaluating the outcome after three years is presented here. Determinants of recurrence were also analysed.

Setting: Department of Dermatology, University Hospital Maastricht, the Netherlands.

Patients and Methods: A randomised controlled trial in 175 primary nBCCs in 150 patients. Primary $n B C C s$ were randomly assigned either to PDT $(n=84)$ group or $S E(n=91)$ group. Tumours treated with PDT were illuminated twice four hours after application of 5-aminolaevulinic acid (ALA) cream. Surgical excision was performed under local anaesthesia with a standard $3 \mathrm{~mm}$ margin followed by histopathological examination.

Main outcome measures: The complete response rate at three months after both the treatments and the disease-free rates at 12, 24 and 36 months of follow-up were assessed.

Results: The median follow-up period was 21.4 months. Incomplete responses or recurrences were observed in $16 \mathrm{BCCs}$ after PDT and in one after SE. The overall 36 months disease-free probability based on the Kaplan-Meier survival analysis was $66.5 \%$ for fractionated PDT and $98.8 \%$ for SE ( $p<0.001)$

Conclusion: The analysis of the results reflects the preliminary outcome comparing fractionated ALA-PDT with SE for treating primary BCCs in a randomised controlled trial. The observed differences in the results were statistically significant with SE being more effective than fractionated ALA-PDT. Although the results of five years of follow-up should be awaited prior to any definite conclusion, in our opinion, SE is preferable to fractionated ALA-PDT for treating primary $n B C C$. 


\section{Introduction}

Although the use of photodynamic therapy (PDT) is rapidly increasing in dermatology, the need for randomised controlled trials (RCTs) to assess its efficacy in basal cell carcinoma (BCC) and other indications is growing. The efficacy of PDT in the treatment of superficial basal cell carcinoma (sBCC) was reported in several RCTs, with complete clearance rates varying from 87 to $94 \%,{ }^{229.248-250}$ The results of PDT treatment in nodular basal cell carcinomas ( $\mathrm{nBCC}$ ) were less promising, with a weighted average complete clearance rates of $53 \%$ in twelve recent studies as reported in a recent update. ${ }^{248}$ Methods to improve the outcome of PDT in $\mathrm{nBCC}$ are debulking of the tumour prior to treatment, repeated treatments and fractionation of the illumination. ${ }^{152-154.251}$

The aim of this study was to compare double illumination ALA-PDT with surgical excision (SE) in nBCC with respect to the efficacy as expressed by long-term disease-free rates. Secondly, determinants of recurrence in the study population were analysed.

The pain that accompanies PDT was also analysed and was been published recently. ${ }^{252}$ The follow-up analysis reflects the preliminary results of this trial with continued followup.

\section{Materials and Methods Patients}

A total of 150 patients with $175 \mathrm{nBCCs}$ were recruited at the outpatient department of dermatology of the University Hospital in Maastricht, the Netherlands between August 2002 and February 2006. All patients older than 18 years, with previously untreated, histopathologically proven BCC of the nodular type were eligible according to the following criteria: localisation anywhere on the body except the eyelids, the lateral corner of the eyes and the ears, clinical size of up to $20 \mathrm{~mm}$ in largest diameter.

Exclusion criteria comprised: recurrent $B C C$, pigmented $B C C$, histological subtypes other than nodular, BCC-nevus syndrome, patients with hypersensitivity to red light or to the 5-ALA cream, patients with protoporphyrea, severe liver/kidney or cardiovascular failure, manifest malignancy, phototoxic/-sensitive drugs and pregnancy. Informed written consent was obtained from all patients. The $\mathrm{nBCC}$ s were randomly assigned to either SE or PDT treatment group once informed consent had been obtained. Randomisation was performed centrally via telephone by a person who was not involved in the trial using a computer-generated random allocation scheme.

Since the practical execution of both treatments differs to a large extent, blinding with respect to the patients or appeared not to be possible. However, when possible, dermatologists other than the research physician conducted the oncological follow-up. 


\section{Treatment procedures}

Photodynamic therapy (PDT)

Three weeks prior to PDT, all patients underwent partial tumour debulking under local anaesthesia. All tumour tissue above the level of the skin was removed by curettage with a Stiefel's sharp curette (no. 4). On the day of the treatment, a felt ring was stuck around the tumour and $20 \%$ 5-aminolaevulinic acid (5-ALA) in Neribas cream was applied including a $5 \mathrm{~mm}$ margin. The area was then covered with occlusive polyurethane dressing (Tegaderm®) and a light protection layer (tinfoil) and was fixed with a bandage. After 4 hours, the area was illuminated with a broadband metal-halogen light source (585-720 $\mathrm{nm})$ either with Medeikonos ${ }^{\star}$ AB (Göteborg, Sweden) or; Waldmann PDT $1200^{\star}$ (Waldmann Medical Technique, Munich, Germany) with an intensity of $100 \mathrm{mwatt} / \mathrm{cm}^{2}$ and a light dose of $75 \mathrm{~J} / \mathrm{cm}^{2}$, after which the treated area was covered again. The area was subsequently re-illuminated after 60 minutes. These two light sources with comparable wavelengths (Medeikonos $^{\star}$ and Waldmann PDT 1200 ) are currently used in daily practice were both used without randomisation. Patients treated with PDT always received both illuminations from the same light source.

\section{Surgical excision (SE)}

Anaesthesia was achieved by a local injection of lidocaine $1 \%$ with epinephrine. The tumour including a $3 \mathrm{~mm}$ margin, which was carefully drawn onto the skin, was excised. ${ }^{82}$ The defect was primareily closed, either by suturing in different layers, or by transposition or transplantation techniques depending on the localisation and the size of the final defect. Routine histopathological examination of Haematoxylin-Eosin stained sections of the lateral and deep margins was performed in the first week to determine whether the excision was complete, taking into account the shortcomings of this so-called bread loaf technique. ${ }^{88}$ In case of positive margins, one or more re-excisions were performed until all margins were free of tumour.

\section{Follow-up}

Follow-up visits took place on day seven in case of SE (removal of the stitches). According to the formal recommendation by the Dutch Society of Dermatology and Venereology, subsequently at $3,6,12,18$ months, and after $2,3,4$ and five years, all patients were examined for recurrent tumours. If necessary, patients had extra check up of the wound in the first month. Incompleteness of PDT was evaluated clinically and confirmed histologically. Incompleteness of SE was evaluated by histological examination. A recurrent tumour was defined as a histologically confirmed $\mathrm{BCC}$ within $5 \mathrm{~mm}$ from the scar. ${ }^{79}$ Areas treated by PDT with no visible tumour tissue during the first control visit after three months, were considered to be treated effectively. Both incomplete responses and recurrences were re- 
treated, either by SE or by Mohs' micrographic surgery (MMS).

Patients lost to follow-up or patients who died during the trial were censored at the date of the last examination.

\section{Statistical analysis}

Data were analysed using SPSS-PC for Windows version 12.0. Assuming that an estimated $95 \%$ of lesions would show complete response to SE, and that the response to PDT would be the same in this study population, it was estimated that approximately 80 lesions per group would be required to demonstrate with $95 \%$ confidence and a power of $80 \%$ that PDT treatment was no more than $10 \%$ inferior to SE treatment. The $10 \%$ difference was agreed on as clinically relevant by the dermatologists who participated in this study. Taking into account an estimated loss of $15 \%$ in the follow-up, we aimed at including 175 tumours.

The interim analysis was performed according to the intention to treat principle.

The cumulative probability of disease-free lesions in both treatment groups were compared using a Kaplan-Meier survival analysis and the log rank test, which computes the number of disease-free lesions at each of the time points. A p-value of 0.05 or less was considered to indicate statistical significance.

The Pearson Chi-Square $\left(X^{2}\right)$ was used to determine a significant difference in the effectiveness of both treatment modalities at three months of follow-up.

A Cox regression model was used to assess the independent effect of treatment modality and other predictors on the recurrence rate. These independent effects are expressed in terms of hazard ratios (HR) and corresponding $95 \%$ confidence intervals $(95 \% \mathrm{Cl})$.

\section{Results}

\section{Patient and tumour characteristics}

A total of 169 primary nBCCs in 147 patients were treated between August 2002 and February 2006 . Men and women were equally randomised (73 vs. 74 ). The mean age of the patients at the time of the treatment was 64.7 years (range 21-91): 63.five years in the ALA-PDT group and 65.8 years in the SE group.

Most of the nBCCs were located on the forehead/temple $(23.4 \%)$, on the dorsum of the trunk $(14.3 \%)$ and on the nose/peri-nasal zone (14.3\%). The tumours had a mean size of $9.1 \mathrm{~mm}$ (SD 3.9, range 2-20) in longest diameter. The distribution and size of the tumours per treatment group are summarised in Table 3.1.

Two BCCs were included from thirteen patients, three BCCs from three patients and four $B C C$ s from one patient were included. The remaining patients all had one single $B C C$ included. Two tumours, allocated to SE group were treated with ALA-PDT and two tumours allocated to ALA-PDT group were treated with SE. 


\begin{tabular}{|c|c|c|c|}
\hline \multicolumn{4}{|c|}{ Number (\%) } \\
\hline & $\begin{array}{l}\text { ALA-PDT } \\
(n=86)\end{array}$ & $\begin{array}{l}S E \\
(n=89)\end{array}$ & $\begin{array}{l}\text { Total no. of lesions } \\
(n=175)\end{array}$ \\
\hline \multicolumn{4}{|l|}{ Location } \\
\hline Forehead/temple & $20(23.3)$ & $21(23.6)$ & $41(23.4)$ \\
\hline Cheek/ chin & $7(8.1)$ & $3(3.4)$ & $10(5.7)$ \\
\hline Nose/ perinasal & $10(11.6)$ & $15(16.9)$ & $25(14.3)$ \\
\hline Lips/ mouth & $1(1.2)$ & $1(1.1)$ & $2(1.1)$ \\
\hline Periocular & $2(2.3)$ & $1(1.1)$ & $3(1.7)$ \\
\hline Periauricular/ ear & $6(7.0)$ & $5(5.6)$ & $11(6.3)$ \\
\hline Neck & $8(9.3)$ & $5(5.6)$ & $13(7.4)$ \\
\hline Trunk ventral & $9(10.5)$ & $11(12.4)$ & $20(11.4)$ \\
\hline Trunk dorsal & $11(12.8)$ & $14(15.7)$ & $25(14.3)$ \\
\hline Extremities & $6(7.0)$ & $7(7.9)$ & $13(7.4)$ \\
\hline Shoulder & $6(7.0)$ & $6(6.7)$ & $12(6.9)$ \\
\hline \multicolumn{4}{|c|}{ Largest diameter (mm) } \\
\hline Mean ((SD)) & $9.1((4.2))$ & $9.0((3.6))$ & $9.1((3.9))$ \\
\hline $2-10$ & $60(69.8)$ & $70(78.7)$ & $130(74.3)$ \\
\hline $11-20$ & $24(27.9)$ & $18(20.2)$ & $42(24)$ \\
\hline Missing & $2(2.3)$ & $1(1.1)$ & $3(1.7)$ \\
\hline
\end{tabular}

Table 3.1 Tumour characteristics

\section{Follow-up}

The median follow-up time was 21.5 months (range 0 - 44), with 118 tumours $(71.1 \%$ ) having a follow-up at least one year. The median follow-up in the ALA-PDT group was 18.5 (range 1-43) months and 24.0 (range 0-44) months in the SE group.

Six tumours dropped out between randomisation and treatment beacause of the following reasons: death which was not related to the skin cancer (1), refusal of treatment after randomisation (2), recurrent $\mathrm{BCC}$ instead of a primary $\mathrm{BCC}(1)$ and double randomisation (2).

At the time of this analysis, three tumours were lost to follow-up directly after treatment and two tumours did not meet the minimum follow-up of three months. Therefore, $164 \mathrm{nBCCs}$ (142 patients) were analysed ( $79 \mathrm{nBBCs}$ treated with ALA-PDT and $85 \mathrm{nBCC}$ s treated with SE). Further losses to follow-up are depicted in the flow chart (Figure 3.1). 


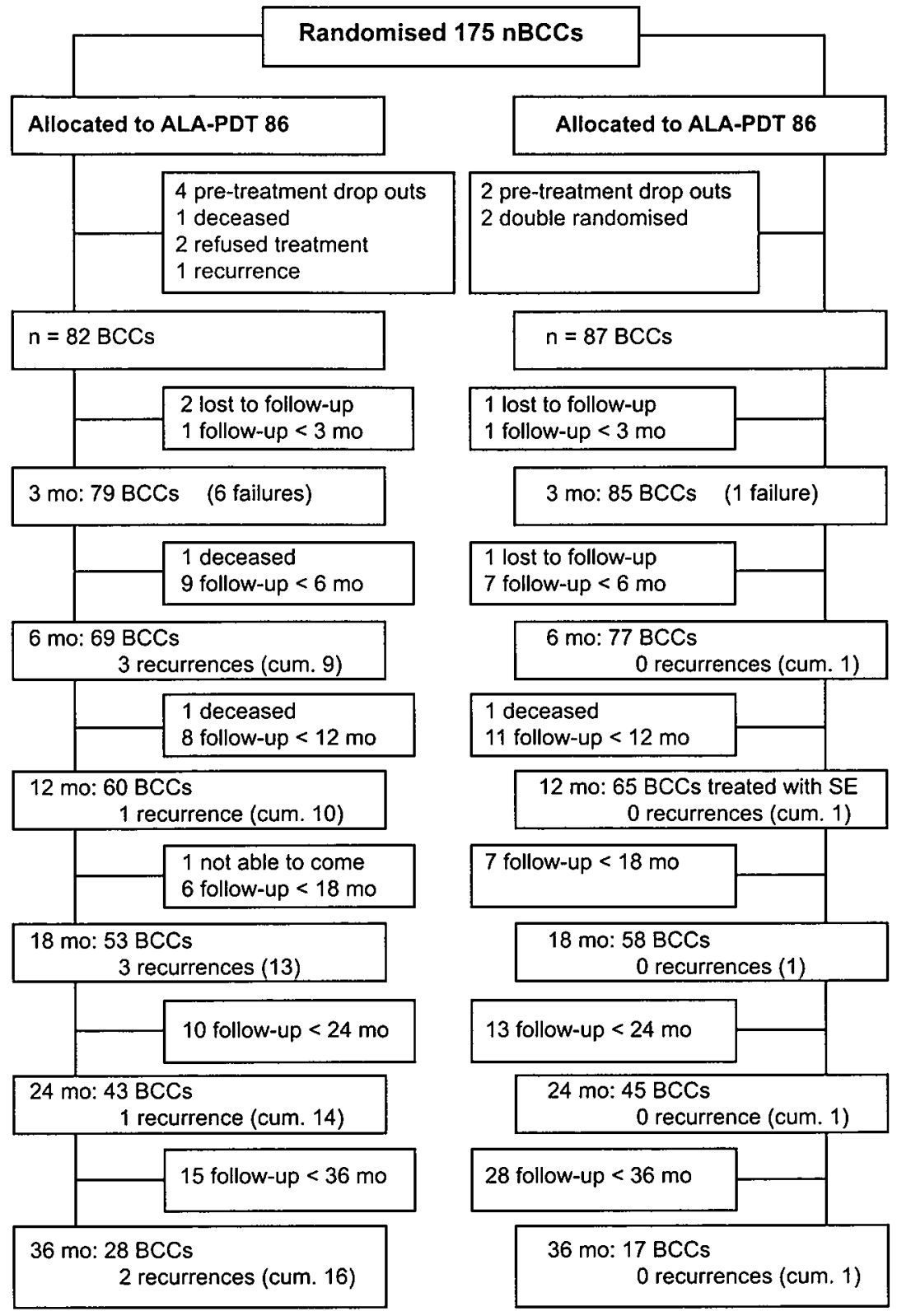

Figure 3.1 Flow chart 


\section{Complete response at 3 months}

At three months 73 out of the $79(92.4 \%)$ nBCCs had resolved completely after treatment with ALA-PDT treatment. In the SE group 84 of the $85(98.8 \%)$ nBCCs had resolved completely $(p=0.042)$. The six lesions, which had resolved incompletely after ALA-PDT treatment were re-treated by SE (4) or MMS (2). The incomplete response after SE was subsequently excised incompletely twice after which MMS was performed.
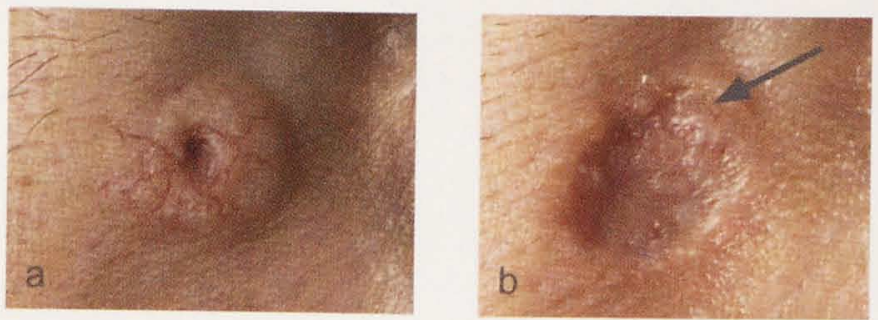

Figure 3.2

a. Clinical appearance of an $\mathrm{nBCC}$ on the nose before treatment with ALA-PDT b. Appearance of the same nBCC 3 months after treatment with residual tumour at the borders (indicated by arrow)

\section{Disease-free rates}

From a total of $125 \mathrm{nBCCs}$ (60 in the ALA-PDT group and 65 in the SE group) that completed one year follow-up, 114 were free of disease. In the ALA-PDT group, four tumours recurred between three months and one year, whereas there were no recurrences in the SE group. The disease-free rate after 12 months was $83.3 \%(50 / 60)$ for PDT vs. 98.5\% $(64 / 65)$ for SE. The disease-free probability rates according to the Kaplan-Meier analysis after 3, 6, 12, 18, 24 and 36 months are shown in Table 3.2.

In total, 16 tumours (of which six incomplete responses) failed to respond to ALA-PDT treatment and SE treatment was unsuccesful in one tumour (incomplete response). The overall 36 months disease-free probability rate based on the Kaplan-Meier survival analysis was $66.5 \%$ for ALA-PDT treatment and $98.8 \%$ for SE treatment $(p<0.001$, Figure 3.3). 


\begin{tabular}{|lcc|}
\hline \multicolumn{3}{|c|}{ Disease-free probability (\%) } \\
$\begin{array}{l}\text { Follow-up time } \\
\text { (mo) }\end{array}$ & PDT & SE \\
3 & & \\
6 & 92.4 & 98.8 \\
12 & 88.0 & 98.8 \\
18 & 86.3 & 98.8 \\
24 & 80.3 & 98.8 \\
36 & 77.6 & 98.8 \\
\hline
\end{tabular}

Table 3.2 Disease-free probability rates (Kaplan-Meier analysis)

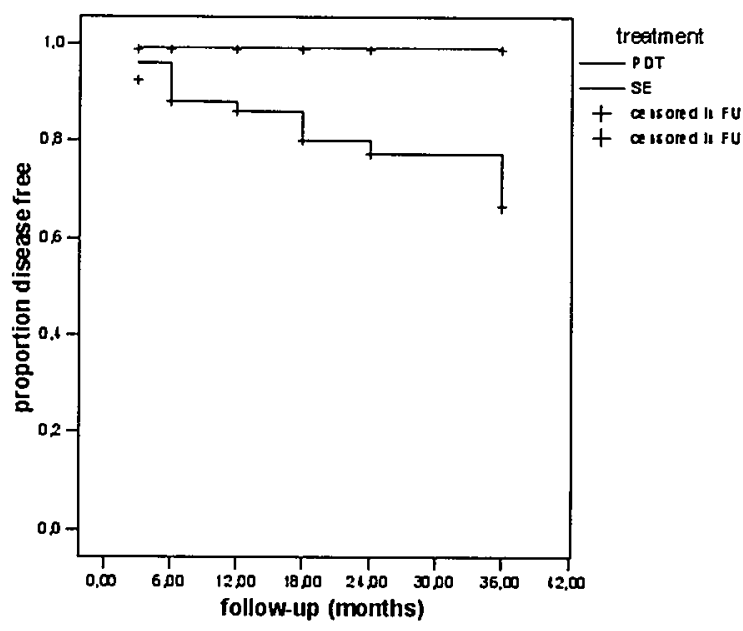

Figure 3.3 Kaplan-Meier survival analysis

\section{Determinants of recurrence}

Using the Cox regression model, without adjustment for prognostic factors, the Hazard Ratio (HR) was 19.07 (95\% $\mathrm{Cl} 2.53-143.90)$, in favour of SE treatment. The HR with adjustment for gender, size, and localisation indicated that the risk of recurrence after ALA-PDT treatment was more than sixteen times higher than after $S E$ treatment $(H R=16.84,95 \%$ Cl: 2.23-127.30).

Location on and around the face led to a HR of 3.13 (95\% $\mathrm{Cl}$ 0.94-9.58). Being male or having a larger size $n B C C$ ( $>10 \mathrm{~mm}$ ) was associated with a two-fold higher risk of recurrence ( $H R=2.69$ and $H R=2.87$, respectively). An age of older than 70 years seemed to be associated with a somewhat lower risk of recurrence $(H R=0.43)$, although it was not statistically significant.

There were no differences in the effectivity of the two light sources that were used. 


\section{Complications}

All side-effects and adverse events of the two treatment modalities were documented, as were the extra visits and measurements that were necessary. Secondary wound infection was observed once after ALA-PDT treatment. No serious complications such as wound dehiscence or necrosis were observed after ALA-PDT or after SE.

\section{Discussion}

To our knowledge, this is a second prospective randomised trial comparing PDT treatment with SE treatment in primary $\mathrm{nBCC}$. Rhodes et al. reported the first prospective, randomised trial in which these treatments were compared for this indication. ${ }^{157}$ However, there were differences in the protocol between their study and this study. These were the use of methyl aminolevulinate (MAL) cream, double treatment with one week interval and wider excision margins. The complete response rates reported by Rhodes et al. did not differ significantly in both tratment groups. At 24 months 19\% (10/53) in the MAL-PDT group and $4 \%(2 / 52)$ in the surgery group had a recurrent BCC. Their results support that MALPDT was as effective as SE for treating $\mathrm{nBCC}$. However, their results after long-term follow-up, may turn in favour of SE. Results of their 36 month follow-up showed an increase in the failure-rate in the MAL-PDT group of up to $21 \%$, including the non-responders. The cosmetic outcome in that study was considerably better in the MAL-PDT-group, which led the authors to recommended MAL-PDT over SE for treating nBCC. ${ }^{253}$

In the present study, at three months follow-up six lesions in the ALA-PDT group showed an incomplete response which was visibly detectable and histologically confirmed. PDT is a non-invasive treatment and therefore may be repeated quite easily. This advantage immediately hosts a disadvantage. In published studies on PDT, many different treatment protocols were used and in several of them the malignancy was re-treated more than once. This makes it difficult, if not impossible to compare the results in these studies. We feel that the intention of all treatments should be complete cure. ${ }^{185}$ Therefore, we chose to treat all nBCCs similarly and only once in this study.

The standard histopathological examination after SE rules out incomplete treatment, whereas after PDT clinicians rely on clinical evaluation. It has been previously reported that this clinical assessment underestimates the number of BCCs with residual tumour. ${ }^{119.250 .254}$ Furthermore, recurrences develop from incompletely responding tumours, which where not recognised at clinical examination. New tumours seldom develop at the treated site. Therefore we choose to approach the incomplete responders and the recurrences as 'failures' and expressed the effectiveness of ALA-PDT as 'disease-free' rates.

In the present study, two different light sources were used. As these were both broadband metal-halogen lamps with a spectral output of between 585 and $720 \mathrm{~nm}$, we did not 


\begin{tabular}{|c|c|c|c|c|c|}
\hline Author & Comparison & $\begin{array}{l}\text { No. of tumours } \\
\text { (No. of patients) }\end{array}$ & Treatment & $\begin{array}{l}\text { follow-up } \\
\text { in months }\end{array}$ & $\begin{array}{l}\text { recurrence } \\
\text { (percentage) }\end{array}$ \\
\hline $\begin{array}{l}\text { Rhodes }{ }^{157} \\
2004\end{array}$ & PDT vs. SE & $118(103)$ & $\begin{array}{l}\text { PDT: } 53 \\
\text { SE: } 52\end{array}$ & 24 & $\begin{array}{l}\text { PDT: } 10(18.9) \\
\text { SE: } 2(3.8)\end{array}$ \\
\hline $\begin{array}{l}\text { Smeets } \\
2004\end{array}$ & MMS vs. SE & $\begin{array}{l}397 \text { primary } \\
201 \text { recurrent }\end{array}$ & $\begin{array}{l}\text { MMS: } 198 \\
\text { SE: } 199 \\
\text { MMS: } 99 \\
\text { SE: } 102\end{array}$ & $\begin{array}{l}30 \\
18\end{array}$ & $\begin{array}{l}\text { MMS: } 3(1.5) \\
\text { SE: } 5(2.5) \\
\text { MMS: } 0(0) \\
\text { SE: } 3(2.9)\end{array}$ \\
\hline $\begin{array}{l}\text { Wang } \\
2001\end{array}$ & PDT vs. CS & $88(77)$ & $\begin{array}{l}\text { PDT: } 44 \\
\text { CS: } 39\end{array}$ & 12 & $\begin{array}{l}\text { PDT: } 11(25) \\
\text { CS: } 6(15.4)\end{array}$ \\
\hline $\begin{array}{l}\text { Avrili255 } \\
1997\end{array}$ & RT vs. SE & $(347)$ & $\begin{array}{l}\text { RT: } 173 \\
\text { SE: } 174\end{array}$ & 48 & $\begin{array}{l}\text { RT: } 11(6.4) \\
\text { SE: } 1(0.6)\end{array}$ \\
\hline $\begin{array}{l}\mathrm{PDT}=\text { Photo } \\
\mathrm{CS}=\text { Cryosu } \\
\text { No.=numbe }\end{array}$ & $\begin{array}{l}\text { mic therapy } \\
y, R T=\text { Radio }\end{array}$ & $\begin{array}{l}=\text { Surgical excis } \\
\text { apy }\end{array}$ & MMS=Moh & rographi & gery, \\
\hline
\end{tabular}

Table 3.3 Randomised controlled trials comparing PDT or SE for BCC to a second treatment

expect to find any differences in the response. In a recently published study on PDT for SBCC ( $n=505)$ where three different light sources were used (of which one diode laser, one broadband metal halogen and one light emitting diode (LED)), no differences in the response were observed. ${ }^{154}$

The efficacy of ALA-PDT and SE in the present study is comparable with that reported in other randomised comtrolled trials in the literature (Table 3.3). The recurrence rates of PDT vary from $18.9 \%$ to $28.6 \%$. Wang et al. compared PDT with cryosurgery. From the patients treated with ALA-PDT 25\% (11 of 44) recurred after one year. ${ }^{119}$ Rhodes et al. reported a disease-free response rate of $83.0 \%$ after treatment with PDT at 12 months follow-up, which is comparable to $86.3 \%$ in the present study ${ }^{157}$

Disease-free probability rate of $66.5 \%$ after 36 months follow-up after ALA-PDT is low compared with that after SE. In our view, the highly significant difference between the two treatment modalities is not expected to alter after follow-up of five years. The difference will also probably not turn in favour of PDT.

In summary, based on the results of this study, we can conclude that ALA-PDT, even with proven effective fractionated illumination ${ }^{154}$, is significant less suitable for treating $n B C C s$. compared with SE. However, we believe that there might be room for ALA-PDT for treating $n B C C s$, for instance in patients who cannot undergo surgery, in patients who desire better cosmetic results of PDT than the risk of incomplete treatment and in basal cell nevus syndrome patients in whom tissue-sparing treatment is essential. ${ }^{256.257}$ Different treatment protocols of PDT may also result in different outcomes. Furthermore, PDT may also be used as a adjuvant or a pre-treatment for surgery or MMS. ${ }^{225,258}$ 


\section{Conclusion}

The preliminary results of this study showed that treatment of $\mathrm{nBCC}$ with SE is significantly more effective than ALA-PDT with double illumination. In our opinion, SE is preferred over ALA-PDT for treating primary $n B C C$ s and we should be reserved with the use of ALA-PDT for this indication based on the large difference in effectivity. Results of five-year follow-up may provide a definite answer. 


\section{Different pain sensations in photodynamic therapy of nodular basal cell carcinoma. Results from a prospective trial and a review of the literature}

Background: Pain is a major side effect of topical photodynamic therapy (PDT), a relatively new and non-invasive treatment for particular types of basal cell carcinoma (BCC). The aim of the study was to characterise in more detail the quality and intensity of pain associated with PDT. Furthermore, the study investigated whether gender, tumour size and localisation as well as different light sources with comparable wavelengths had an influence on the pain.

Methods: A total of 64 nodular BCCs in 55 patients, of which 48 BCCs underwent preceding debulking, were treated with 5-aminolevulinic acid (ALA-PDT). Two light sources, the Medeikonos ${ }^{(8)}$ and the Waldmann PDT $1200^{\star}$, were used at random. Pain assessment was performed using a visual analogue scale (VAS).

Results: All patients experienced pain during illumination and $41.8 \%$ after illumination. The mean pain intensity was 3.88 with most patients experiencing burning $(82.5 \%)$ or stinging $(36.8 \%)$ sensations. Illumination with the Medeikonos ${ }^{\infty}$ light source was experienced as less painful than with the Waldmann ${ }^{\otimes}$ lamp (4.64 vs. 3.40; $p=0.027$ ). Gender as well as tumour localisation and size did not alter the pain scores, and no differences were observed between patients who underwent debulking and those who did not.

Conclusions: Treatment of single BCCs with ALA-PDT rarely resulted in unbearable pain. However, the degree of pain can vary depending on the light source used. Further studies are needed to unravel the patho-mechanisms underlying the development of pain in PDT in order to develop adequate solutions to this undesirable side-effect. 


\section{Introduction}

With the rising incidence of basal cell carcinoma (BCC), the arsenal of treatment modalities is also expanding. Photodynamic therapy (PDT) is a relatively new treatment of basal cell carcinoma. However promising, its position in the spectrum of $\mathrm{BCC}$ treatment has not yet been established.

The therapy is based on activation of a topically applied photosensitiser (light-sensitive molecules). These molecules form various cytotoxic species, which will damage essential cellular components of neoplastic or dysplastic tissue, causing the cells to undergo apoptosis. Other mechanisms of PDT are necrosis by direct damage and targeting of the surrounding vasculature. Furthermore, inflammatory and immune responses contribute to the damaging effect of PDT. ${ }^{138}$ At the cell level, mitochondria, lysosomes, plasma membranes and nuclei of tumour cells have been evaluated as potential PDT targets. ${ }^{259}$

The traditionally most commonly used topical photosensitiser is $20 \% 5$-aminolevulinic acid (ALA) cream that is converted into protoporphyrin IX (PpIX), which is the immediate precursor of haem. The accumulation of $\mathrm{PpIX}$ is increased in tumour tissue, as compared to normal tissue, because of increased permeability in tumour tissue. ${ }^{260-264} \mathrm{~A}$ photosensitiser that is increasingly being used is the commercially available methyl ester of 5-aminolevulinic acid (Metvix ${ }^{\oplus}$, Galderma, Paris).

PDT successfully treats superficial $\mathrm{BCCs}(\mathrm{SBCC})$ with clearance rates ranging from $68 \%$ to $100 \%{ }^{155.230 .249 .265 .266}$ and yields cosmetically excellent results. ${ }^{157,230,263}$ It is a safe procedure with few side effects. One of the advantages of PDT is that large fields of actinic keratosis $(A K)$ or $B C C$ s can be treated in one session, which can be an advantage especially for organ transplant patients, basal cell nevus syndrome patients and patients with multiple BCCs. ${ }^{267,268}$ The use of PDT is also an advantage over surgical treatment in treating areas with poor wound healing, like BCC on the lower extremities and in the elderly. ${ }^{262}$

The photosensitiser is applied with a wide margin around the tumour during PDT, but accumulation of PpIX will take place mainly in the tumour cells. Therefore, in contrast to the more conventional therapies, such as surgical removal, cryotherapy and curettage, there is a selective destruction of the malignant cells with minimal damage to the surrounding healthy tissue. This tumour selectivity is a major advantage in patients with multiple sB$\mathrm{CCs}$, in which as much healthy skin as possible must be spared as it might be needed for future reconstructions. Disadvantages of PDT are the lack of histological control, and the time-consuming aspect. ${ }^{262}$

PDT is contra-indicated in pigmented $\mathrm{BCCs}$, because of diminished penetration of the light in the tumour. However, a recent study has shown that PDT using a wavelength of $390 \mathrm{~nm}$ does penetrate into a pigmented $\mathrm{BCC} .{ }^{269}$ Also contra-indicated is the treatment of $\mathrm{BCCs}$ with histological types other than superficial or nodular $\mathrm{BCC}(\mathrm{nBCC}) .^{262.270}$

The major side effect of PDT, which is described in different studies, is the pain experi- 
enced during treatment. Most patients experience a stinging and/or burning sensation, usually mild or moderate. ${ }^{261.263 .264 .266 .271 .272}$ Occasionally, the pain can be intolerable to certain individuals leading to cessation of the treatment. This is likely to occur more frequently when large fields or facial areas are treated. ${ }^{263}$

Pain can occur both during and after treatment. The underlying mechanism of pain caused by PDT has not been unravelled so far, but several theories have been proposed. These theories will be discussed later in this thesis.

Immediately after illumination, erythema, oedema and crusting are common, which mostly resolves within four weeks after treatment. ${ }^{261,262,270,273}$ Temporary hyperpigmentation is sometimes seen in the illuminated skin. ${ }^{274}$

A literature search has revealed that there are only a limited number of studies concerning the pain experienced during photodynamic therapy. The objective of this study was to learn more about the character and intensity of the pain and to compare this with the reports in the literature. Furthermore, the study was aimed to investigate whether large-size and certain localisations predisposes to more pain. The level of pain experienced from two different light sources with comparable wavelengths, which are both currently in use in PDT practice, was also compared.

\section{Materials and methods Study design}

This study forms part of a randomised controlled trial 'Comparison of fractionated photodynamic therapy with surgical excision in the treatment of basal cell carcinoma; preliminary results of a randomised controlled trial', in which patients with primary nBCCs were randomly treated with either conventional excision or ALA-PDT. The goal of this part of the study was twofold. The primary goal was to obtain the results of the pain assessment that was performed in all BCCs treated with ALA-PDT. Two light sources with comparable wavelengths (Medeikonos ${ }^{\star}$ and Waldmann PDT $1200^{\circledR}$ ), which are currently used in daily practice, were also compred. No formal randomisation was performed.

\section{Patient selection}

Patients between 18 and 85 years of age with previously untreated, histopathologically proven $\mathrm{BCC}$ of the nodular type, were recruited from the outpatient department of Dermatology of the University Hospital in Maastricht, the Netherlands. Patients were selected according to the following criteria: nodular, primary BCC localised anywhere on the body except the eyelids, the lateral and medial corner of the eyes and the ears, with a clinical diameter smaller than $20 \mathrm{~mm}$. The exclusion criteria were recurrent $\mathrm{BCC}$, pigmented $\mathrm{BCC}$, histological subtypes other than nodular, basal cell nevus syndrome, hypersensitivity to red light or to the cream, porphyrea, manifest malignity, phototoxic/photosensitive drugs, 
pregnancy and patients with more than ten BCCs. The medical history of the patient concerning pain medication was carefully noted. Informed written consent was obtained from all patients. Patients were informed about the pain assessment to be performed during treatment.

\section{Treatment procedure}

Partial debulking of the tumour was carried out three weeks before treatment. All tumour tissue apparent above the level of the skin was removed by curettage with a Stiefel ${ }^{\star}$ sharp curette (no. 4). In some cases curettage was not performed if no tumour tissue was observed above the level of the skin.

After sticking a felt ring around the tumour, the 5-ALA cream was applied on the tumour including a 5-mm margin. The whole treated area was then covered with occlusive polyurethane dressing (Tegaderm ${ }^{\circledR}$ ) and a light protection layer (tinfoil). This was then all fixed in place with a bandage. After an incubation time of four hours the bandage and surplus of cream was gently removed after which the area was illuminated with a broadband red light source with an intensity of $100 \mathrm{mWatt} / \mathrm{cm}^{2}$ and a light dose of $75 \mathrm{~J} / \mathrm{cm}^{2}$. Two different broadband light sources that were available in the department (Medeikonos ${ }^{\circledR} \mathrm{AB}$, Göteborg, Sweden; Waldmann PDT $1200^{\circ}$, Waldmann Medical Technique, Munich, Germany), were used. Additional details of these are given in Figure 3.4.

\section{Pain assessment}

A visual analogue scale (VAS) was used with scores from $0-10$, with 0 indicating no pain, and 10 indicating unbearable pain, was used to measure the pain intensity experienced by the patients during the PDT. ${ }^{275}$ During or directly after treatment the patients were asked to score the experienced pain during the illumination as well as the character, duration and radiation of the pain. The character of the pain was rated as burning, stinging, throbbing, tingling or lingering. Additional answers were possible. Duration of the pain was assessed during and after application of the cream, and during and after illumination. Additional answers were also possible for describing the duration. Finally, patients were asked if there was any sign of radiation of the pain into the surrounding tissue.

\section{Statistical analysis}

Data was analysed using SPSS-PC for Windows version 11.0. Mean pain intensity scores between groups were compared using the independent Samples T-test or ANOVA. A pvalue of 0.05 or less was considered to indicate statistical significance. 


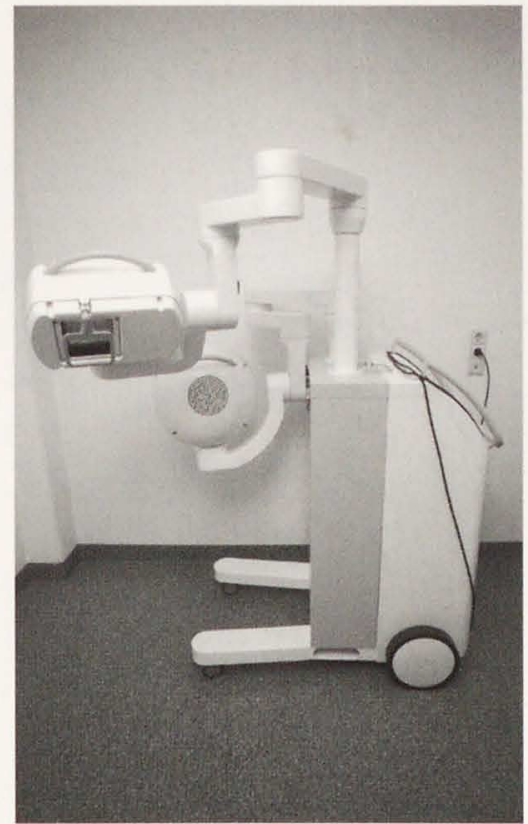

Medeikonos

Source

Treatment dose

Light intensity

Wavelength

Treatment area
Metal halogen

$10-150 \mathrm{~J} / \mathrm{cm}^{2}$

$40-100 \mathrm{~mW} / \mathrm{cm}^{2}$

$580-680 \mathrm{~nm}$

$10-10 \mathrm{~cm}$

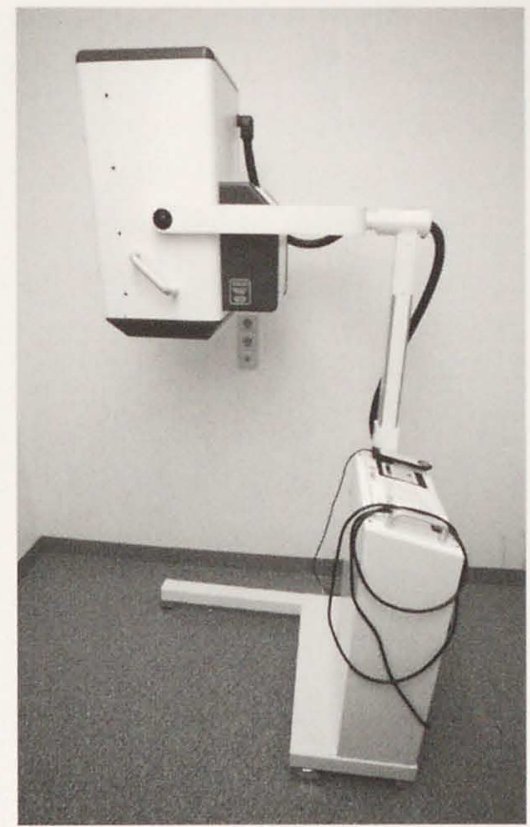

Waldmann PDT 1200

Metal halogen

$10-200 \mathrm{~J} / \mathrm{cm}^{2}$

$10-200 \mathrm{~mW} / \mathrm{cm}^{2}$

$600-750 \mathrm{~nm}$

$\varnothing 15 \mathrm{~cm}$

Figure 3.4 Specifications light sources

\section{Results}

A total of $68 \mathrm{BCCs}$ were treated in 60 patients. More men than women were treated $(33 / 60$; $55 \%$ vs. $27 / 60 ; 45 \%$ ). In total, 64 BCCs were analysed (one patient died before treatment and three patients were excluded from the study before receiving treatment because they were found not to meet the inclusion criteria). The average age of the patients at the time of treatment was 63.3 years (SD 13.8; range 24-83). Two patients used pain medication (Ibuprofen) on daily basis for a medical condition not related to BCC.

The mean size of the tumours was $8.95 \mathrm{~mm}(95 \% \mathrm{Cl} 9.94-7.97$; SD 3.95; range 3-20) by $7.52 \mathrm{~mm}(95 \% \mathrm{Cl}$ 8.33-6.72; SD 3.22; range 3-20). Most of the tumours were located in the face $(33 / 62 ; 53.2 \%)$. Five BCCs were located in the neck area $(8.1 \%)$, eight on the trunk on the anterior side (12.9\%), eleven on the back (17.7\%) and five on the lower extremities (8.1\%) (Figure 3.5). Most BCCs were treated with the Medeikonos lamp $(40 / 64 ; 62.5 \%)$ and 23 tumours were treated with the Waldmann PDT 1200 lamp (23/64; 35.9\%). This information was lacking for one tumour. The distribution and size of the tumours were comparable between these two groups. 
Pain scores (VAS) were completed in 57 cases (89.1\%). The mean pain intensity was 3.88 (SD2.07; range 1-8). There were no patients who discontinued the treatment. Figure 3.6 shows the VAS scores in a histogram.

When describing the character of the pain, 35 patients selected one, 20 patients selected two, and 2 patients selected three possibilities. In most cases the pain was experienced as burning ( $n=47 ; 82.5 \%)$, in 21 cases (36.8\%) as stinging, in 9 cases (15.8\%) as tingling and in only a few cases as lingering $(n=3 ; 5.3 \%)$ or throbbing $(n=1 ; 1.8 \%)$. When describing the duration of pain 30 patients selected one, 24 patients selected two, and 1 patient selected three possibilities. All patients experienced the pain particularly during the illumination, $41.8 \%(n=23)$ of the patients also experienced the pain after illumination and only a few patients also experienced the pain during $(n=1 ; 1.8 \%)$ or after application of the cream $(n=2 ; 3.6 \%)$. The question whether radiation of pain existed was answered by $55(85.9 \%)$ patients. In most cases, no radiation was mentioned, but in some cases $(4 / 55 ; 7.3 \%)$ radiation into the direct surroundings was reported. These tumours were all located in the face, two on the cheek, one on the upper lip and one on the nose.

The mean pain score for men (3.63; SD 2.08) was not statistically significantly different from the mean pain score for women (4.15; SD2.07; $p=0.353$ ).

A difference in favour of the Medeikonos ${ }^{(i)}$ device (3.40; SD 2.19 versus 4.64; SD 1.65) was found when two broadband light sources were compared $(p=0.027)$.

The pain intensity of patients in relation to their age was also considered. The patients were dichotomised by age. The mean pain score for patients under $65(n=30)$ was 3.70 (SD1.78) and the mean pain score for patients older than 65 years $(n=27)$ was 4.07 (SD 2.37). There was no statistically significant difference between the two groups $(p=0.501)$.

No statistically significant difference was found between the mean pain score from patients who were treated in the facial/neck area ( $n=34$; mean 3.97 ; SD 2.21) and patients who were treated on the trunk or extremities $(n=21$; mean $3.67 ;$ SD $1.96 ; p=0.607)$. Of all localisations regarded separately, only the nose seems to be more painful (mean 5.83 ) than other areas.

Patients were also divided into two groups based on the size of the tumour. No statistically significant difference between the mean pain score from patients with small tumours (1-10 $\mathrm{mm} ; \mathrm{n}=40$; mean 4.10; SD2.06) and patients with large tumours ( $>10 \mathrm{~mm} ; \mathrm{n}=15$; mean 3.20; SD 2.15; $p=0.159$ ) was found.

Prior to treatment 48 tumours underwent tumour debulking: this was not performed for eight tumours, and in one case, this information was lacking. The mean pain score for the patients that underwent debulking (3.88; SD 2.12) was not statistically significant different $(p=0.877)$ from the mean pain score for the patients who were not curetted $(3.75 ;$ SD 1.98). In order to investigate whether there is a relation between the pain and the efficacy of the treatment, the pain scores of the patients in which the tumour was not cleared completely 
or had recurred $(n=9)$ was analysed. The mean pain score in these patients was 3.50 (SD 2.33) compared to a mean pain score of 4.07 (SD 2.12) in the patients that had no recurrence. This difference did not reach a statistically significance level $(p=0.495)$.
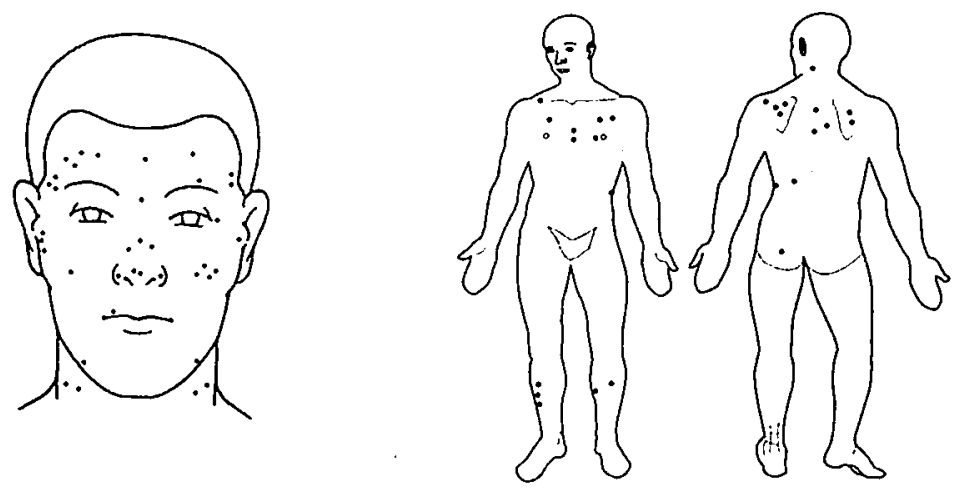

Figure 3.5 Distribution of tumours

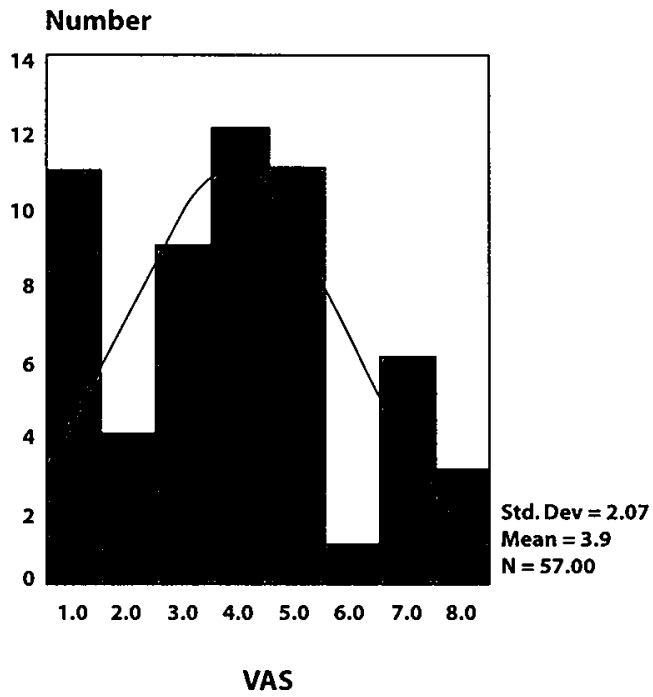

Figure 3.6 Distribution of VAS scores 


\section{Discussion}

Pain is considered to be a major side effect of ALA-PDT for BCCs and AK. The aim of the study was to objectify the pain experienced during ALA-PDT treatment, compare this to the reports in the literature and investigate possible influencing variables. The pain that was experienced in the treatment of $57 \mathrm{BCC}$ was assessed. The pain intensity experienced in this study, on a VAS-scale from 0 to 10 , had a mean value of 3.88. This mean VAS score during irradiation is comparable with rates of 2.30 to 4.38 reported in other studies (Table 3.4). The group of patients who received ALA-PDT in this study was much larger compared to those other studies. It is difficult to interpret the pain experienced by patients during PDT because pain is a rather subjective feeling and because of the large inter-patient variation. Nonetheless it is apparent from this study that ALA-PDT never resulted in unbearable pain when used on single, nodular BCCs.

As described in the literature, most patients experienced the pain as burning and some also as stinging. All patients experienced the pain during illumination and some also did directly after treatment. Only a few patients experienced radiation of the pain, but this was not distributed within a dermatome or in the area of a large nerve.

Interestingly, the results imply that lower pain intensities are experienced when the Medeikonos ${ }^{\text {ight }}$ ligurce is used. This has not been described in the literature before, as different light sources with comparable wavelengths have not been previously compared in pain sensation studies. The difference in pain intensity between the two light sources cannot be explained by differences in wavelength or the size of the illumination field, as they are comparable. The only difference is that Medeikonos ${ }^{5}$ has an internal ventilator for cooling of the apparatus. It would seem valuable in the future, to compare pain induced by PDT with the metal halogen broadband light sources to the newer generation of light emitting diode (LED) -based light sources.

There were also no differences in localisation, size or debulking of the tumour between the two groups.

In contrast to the study of Grapengiesser et al. ${ }^{263}$, no significant difference between the pain experienced during photodynamic treatment of facial lesions compared to PDT of lesions on the trunk or extremities could be detected. Possibly, the number of patients treated with PDT in this study was too small, although it was still larger than in studies where a difference was recorded. It does seem that ALA-PDT is more painful on the nose $(n=6$; mean 5.83 ; SD 2.56) but no strong conclusions can be drawn because the number of patients is too small to perform a proper statistical analysis.

In theory, treatment of large tumour areas with PDT is likely to lead to more pain than treatment of smaller areas, as was found in another study. ${ }^{263}$ No statistically significant difference between treatment of small and larger lesions was found in this study. This may possibly be explained by the fact that in this study only relatively small BCCs (up to 20 
$\mathrm{mm}$ diameter) were treated. More comparative studies are needed to investigate whether treatment of larger lesions is more painful.

There seems to be no difference in pain experience between men and women, in contrast to Grapengiesser et al. ${ }^{263}$ who used Waldmann PDT $1200^{*}$ as well. In their study men experienced more pain than women. In the present study, a somewhat higher pain score was found in women, although not statistically significant.

No statistically significant difference was found when comparing the pain scores of patients who underwent debulking before treatment and those who did not receive curettage. The debulking was performed three weeks before ALA-PDT and in this period the wounds resulting from the debulking procedures are likely to have healed. Therefore, possible differences in pain scores cannot be explained by the application of an acid cream onto a fresh wound.

PDT is often regarded as a painful procedure. In some cases it requires interventions for pain control including application of ice, use of a fan, interruption of treatment, forced cooled air, and topical and oral analgesia. ${ }^{261.272 .273}$ Pain seems to be more of an issue with PDT than it does with other treatments of BCCs. The fact that pain in other treatments occurs at other stages of the procedure is probably often overlooked. Pain is experienced with other treatments, such as conventional excision, while giving the anaesthesia. Some patients also experience the removal of the sutures as painful. Perhaps less pain is expected, and thus more easily accepted, in non-invasive treatments compared to invasive treatments where pain is expected. Rhodes et al. ${ }^{157}$ compared PDT with conventional excision, but did not quantify the intensity of pain on a standardised pain score. They found that, unlike surgery, there was no routine need for local anaesthesia. The pain with PDT was described as mild or moderate and resolving the same day without the need for medical intervention. Comparative studies are needed, in which pain in PDT is compared to pain in other treatments, preferably with intra-patient (left-right) comparisons, in order to compare not only efficacy but also side-effects such as pain.

The experience of the authors is that explanation of the procedure to the patients and reassurance is helpful. Patients may be anxious about a new treatment and therefore more susceptible to pain. It is often stated that patients contributed the pain they experienced to the heat produced by the lamp. A study by Orenstein et al. showed that irradiation of normal skin without ALA application was not accompanied by any pain even when the temperature was $44^{\circ} \mathrm{C}$ to $45^{\circ} \mathrm{C} .^{276}$

Pain during treatment is supposed to be a result of a combination of intense nerve stimulation by reactive oxygen species and hyperthermia. ${ }^{273}$ Another theory is that ALA is taken up by $\gamma$-aminobuturic acid (GABA) transporters into the peripheral nerve endings. ${ }^{264.277}$ The discomfort post-treatment is more typical of an inflammatory process. ${ }^{270}$ Treatment of 
large tumour areas and well-innervated anatomical locations such as the face and hands seems to result in more intense pain than treatment of smaller areas or other locations. Also, treating actinic keratosis is reported to be more painful than treating BCC.

Unlike ALA, methyl ester aminolevulinic acid (MAL) is not transported by GABA transporters into the peripheral nerve endings. This may explain the clinical experience of more intense pain reactions observed during PDT after application of ALA as compared to MAL ${ }^{264}$, which has also been confirmed in a recent study. ${ }^{254}$

More research is required to unravel the mechanism of pain in photodynamic therapy and to search for adequate solutions in combating this problem. Only nBCCs were included in this study, but it may be of great value to examine sBCCs as well in order to unravel the pain mechanism.

\section{Conclusion}

Patients experience pain when treated with PDT. The intensity of the pain experienced by patients in this study ranged from 3.5 to 4.6 on a VAS scale from 0 to 10 . This is comparable with earlier findings reported in the literature.

Single $\mathrm{nBCCs}$ up to $20 \mathrm{~mm}$ with prior tumour debulking can be well treated with photodynamic therapy without cessation of the treatment due to pain.

No statistical difference was found in pain intensity that could be attributed to the size and localisation of the tumours. PDT with the Medeikonos ${ }^{(i)}$ light source appeared to be less painful than with the Waldmann PDT $1200^{\circ}$ light source. More studies are needed to confirm any differences between various light sources.

While PDT is often regarded as a painful treatment, there is not much knowledge how this pain compares to that caused by other treatments. Comparative studies are needed to establish this.

The underlying mechanism of pain caused by PDT has not been elucidated so far. More studies are needed so that more insight into this mechanism may lead to a possible analgesic solution for PDT. 


\begin{tabular}{|c|c|c|c|c|c|c|c|}
\hline Author & $\mathrm{N}$ & $\begin{array}{l}\text { Photo } \\
\text {-sensitiser }\end{array}$ & $\begin{array}{l}\text { Illumination } \\
\left(\mathrm{mW} / \mathrm{cm}^{-2}\right)\end{array}$ & VAS & Lesions & Analgesia & Other findings \\
\hline Grappengiesser ${ }^{263}$ & 31 & ALA & $50-130$ & 3.5 & $\mathrm{BCC}$ & EMLA & $\begin{array}{l}\text { Larger lesions } 2002 \\
\text { more painful } \\
\text { Lesions on head } \\
\text { more painful }\end{array}$ \\
\hline $\begin{array}{l}\text { Wiegel|1264 } \\
2003\end{array}$ & 20 & $\begin{array}{l}\text { ALA } \\
\text { MAL }\end{array}$ & 90 & $\begin{array}{l}4.2 \\
2.7\end{array}$ & $\begin{array}{l}\text { Normal } \\
\text { skin }\end{array}$ & - & $\begin{array}{l}\text { ALA more painful } \\
\text { than MAL }\end{array}$ \\
\hline $\begin{array}{l}\text { Ericson }{ }^{278} \\
2004\end{array}$ & 32 & ALA & $\begin{array}{l}30 \\
45 \\
50 \\
75\end{array}$ & $\begin{array}{l}2.9 \\
3.5 \\
2.8 \\
3.6\end{array}$ & AK & - & $\begin{array}{l}\text { No difference } \\
\text { between fluence } \\
\text { rates }\end{array}$ \\
\hline $\begin{array}{l}\text { Holmes }{ }^{272} \\
2004\end{array}$ & 24 & ALA & $70-90$ & $\begin{array}{l}4 \\
4.5\end{array}$ & $\begin{array}{l}\mathrm{BCC}, \mathrm{AK} \\
\text { and } \mathrm{BD}\end{array}$ & $\begin{array}{l}\text { Tetracain } \\
\text { Placebo }\end{array}$ & $\begin{array}{l}\text { No effect of } \\
\text { tetracain gel }\end{array}$ \\
\hline $\begin{array}{l}\text { Kuijpers } 254 \\
\text { In progress }\end{array}$ & 32 & $\begin{array}{l}\text { ALA } \\
\text { MAL }\end{array}$ & 100 & $\begin{array}{l}4.38 \\
2.84\end{array}$ & $\mathrm{BCC}$ & - & - \\
\hline Present study & 57 & ALA & 100 & 3.88 & $\begin{array}{l}\text { Nodular } \\
\text { BCC }\end{array}$ & - & $\begin{array}{l}\text { Medeikonos light } \\
\text { source less painful } \\
\text { than Waidmann } \\
\text { PDT1200 }\end{array}$ \\
\hline
\end{tabular}

$\mathrm{ALA}=$ Deltaaminolevulinic acid, $\mathrm{MAL}=$ Methylaminolevulinate, $\mathrm{BCC}=\mathrm{Basal}$ cell carcinoma, AK=Actinic keratosis, Emla=Ointment containing prilocain and lidocain

Table 3.4 Studies reporting on pain intensity in PDT 
3.3

\section{Similar effectiveness of methyl aminolevulinate and 5-aminolevulinate in topical photodynamic therapy for nodular basal cell carcinoma}

Background: Photodynamic therapy (PDT) for basal cell carcinoma (BCC) is a treatment modality, the application of which is expanding within the dermato-oncology. Currently, two topical photosensitisers, 5-aminolevulininate (ALA) and methyl aminolevulinate (MAL) are widely used, but their efficacy in $B C C$ has not yet been compared in a clinical setting.

Objective: To evaluate the effect, degree of pain, and costs of ALA-PDT versus MAL-PDT in nodular BCC.

Methods: Patients suffering from BCC were randomly assigned to either ALA-PDT $(n=22)$ or MAL-PDT $(n=21)$ in a pilot study. In each group, half of the tumours were debulked prior to PDT. The therapeutic outcome was checked histologically eight weeks after treatment. Pain scores were assessed using a visual analogue scale.

Results: Residual tumour tissue was detected in six BCCs of each study group. No significant difference between ALA-PDT and MAL-PDT was found with regard to therapeutic efficacy and pain scores. Notable, however, was that the costs of MAL-PDT were six times higher than those of ALA-PDT.

Conclusion: No differences in the short-term efficacy were found in this study between ALA-PDT and MAL-PDT. Therefore, both ALA and MAL can be equally recommended as topical photosensitisers in PDT. 


\section{Introduction}

Photodynamic therapy (PDT) is a relatively novel but promising treatment of basal cell carcinoma $(B C C)$, which generally has little side effects, although pain can be a limiting factor especially when treating large areas.

It has proven effective for superficial BCC (sBCC) with complete response rates of $68 \%$ to $100 \%$, and compared to surgical excision, less invasive and with better cosmetic results. ${ }^{119,155,249,265,266}$ The response of nodular BCC (nBCC) to topical PDT is less favourable, with initial complete responses varying from $50 \%$ to $91 \%$ when treated with double illumination schemes. ${ }^{157.249,279}$ Insufficient penetration of the topically applied 5-aminolevulinic acid (5-ALA) into the tumour has been suggested as a possible explanation for these relatively poor results with nodular $\mathrm{BCC}(\mathrm{nBCC}) .{ }^{152}$ Various methods of dealing with this problem have been proposed, such as the use of tissue enhancers such as dimethylsulfoxide (DMSO), PpIX accumulating edetic acid (EDTA), prior curettage, changes in the formulation of the ALA vehicle and drug delivery via liposomes and macromolecules. ${ }^{153,280,281}$ A different approach is the use of more lipophilic derivates of ALA, such as ALA methyl and alkyl esters. Pre-clinical studies have shown more favourable pharmacological properties with respect to PpIX formation, tissue penetration and tissue selectivity for the esters.

5-ALA is licensed in the USA for the treatment of solar keratosis (Levulan Kerastick ${ }^{\circledR}$, DUSA Pharmaceuticals, Wilmington, USA). The methyl ester ALA (MAL) has become commercially available in Europe $\left(160 \mathrm{mg} / \mathrm{g}\right.$ Metvix ${ }^{\oplus}$, Galderma, Paris, France and Photocure, Oslo, Norway) for the treatment of superficial and nodular BCC and solar keratosis. Worldwide, dermatologists have their preferences for one or other of these sensitisers, based on expectations of efficacy, usefulness, costs and adverse events (e.g. pain). So far, no clinical studies comparing these sensitisers can be found in the literature. This study compares PDT with ALA and MAL for $\mathrm{nBCC}$ with respect to the criteria mentioned above.

\section{Material and methods Study design}

The study was designed as a pilot study, using excision with histopathological control eight weeks after PDT to determine the efficacy of the treatment and to overcome the long waiting time of long-term follow-up after this moment. The tumours were allocated to either ALA-PDT or MAL-PDT. In each group, half of the numbers of tumours were pre-treated with tumour debulking, directly followed by the photodynamic procedure, with similar illumination protocols. 


\section{Patient selection}

Patients were recruited from the outpatient department of dermatology of the University Hospital in Maastricht, the Netherlands, according to the following inclusion criteria: nodular, primary $\mathrm{BCC}$, localised anywhere on the skin but the peri-ocular area and hairy scalp, with a clinical diameter smaller than $20 \mathrm{~mm}$. The exclusion criteria were pigmented BCC, contra-indications to surgery, hypersensitivity to daylight or to either of the creams, patients with any type of porphyrea, and patients with more than five BCCs. After the patients had given informed consent, the BCCs were randomly allocated to either of the precursors. The patients were blinded to the treatment regarding the used precursor. Photographs documented selected tumours and surroundings.

\section{Treatment protocol}

All tumour tissue arising above the level of the skin was removed by curettage with a Stiefel's sharp curette (no.4) after topical anaesthesia with ethyl chloride spray (Coolspray ${ }^{\otimes}$ ) for patients in a debulking group. The visible tumour including a $5 \mathrm{~mm}$ margin was covered by either $16 \%$ MAL cream (Metvix ${ }^{\oplus}$, Galderma, Paris, France), or by $20 \%$ 5-ALA (Sigma, Germany) in cream (w/o) (Neribas, Schering, the Netherlands) in a non-transparent layer with a thickness of approximately $2 \mathrm{~mm}$. The latter was freshly prepared by the hospital pharmacist. The whole was then covered with polyurethane dressing (Tegaderm ${ }^{\odot}$, $3 \mathrm{M}$, Zoeterwoude, the Netherlands) and an opaque dressing. After an incubation time of three hours, any excess cream was removed, and the area was gently cleaned using saline and illuminated with a broadband, metal halogen, light source $(600-730 \mathrm{~nm}$ ) (Waldmann PDT1200, Waldmann Medical Technique, Munich, Germany) with an intensity of $100 \mathrm{mWatt} / \mathrm{cm}^{2}$ and a total, single, light dose of $75 \mathrm{~J} / \mathrm{cm}^{2} .{ }^{282}$ After illumination, the treated area was covered with a light protective dressing during 24 hours, and in case of pain, treated with a topical emollient.

The treatment protocol as described above was repeated after seven days with the same precursors, but this time without tumour debulking. ${ }^{283}$ Eight weeks after the second treatment cycle, the treated tumour area including a safety of $3 \mathrm{~mm}$, was surgically excised. Delineation of this area was performed on visual inspection based on the present erythema in comparison to the photographs. The excised specimen was examined histopathologically with haematoxylin and eosin staining to detect tumour remnants. The pathologist examining the sections was blinded to the treatment.

\section{Pain assessment}

Patients were asked for the intensity, character, duration and radiation of the pain. A visual analogue scale (VAS) with scores ranging from 0-10 - with 0 indicating no pain at all and 10 indicating unbearable pain - was used for the pain intensity. The character of the pain 
was rated as burning, stinging, throbbing, tingling or lingering. Combined answers were possible. Duration of the pain was assessed during and after application of the cream, and during and after illumination. Finally, patients were asked for occurrence of radiation of the pain.

\section{Statistical analysis}

Data was analysed using SPSS-PC for Windows version 11.0. Evaluation took place by comparing the proportions of patients without complete clearance, using the Pearson ChiSquare test. Mean pain intensity scores between groups were compared using the Independent Samples T-test. Pain intensities at two moments within the same patients were compared using the paired Samples T-test. A p-value of 0.05 or less was considered to indicate statistical significance.

\section{Results}

A total of $43 \mathrm{BCCs}$ in 39 patients were treated. More men than women were treated (24 vs. 15). The average age of the patients at the time of treatment was 68.4 years (median 73, range 39-87). The baseline characteristics of the treatment groups were comparable (Table 3.5).

Twenty-two (51\%) tumours were treated with ALA-PDT (group 1) and 21 (49\%) with MALPDT (group 2). Tumours within the ALA and MAL group, $11(50 \%)$ and $10(48 \%)$ respectively, underwent debulking prior to treatment.

In total, tumour remnants were found in twelve cases $(12 / 43,28 \%)$. The incomplete clearance rates per treatment group are shown in Table 3.6.

Pain scores were completed in 32 cases (82\%) during the first treatment cycle (Table $3.7)$. In most cases the character of the pain was assessed as burning (59\%) or stinging $(41 \%)$, less frequently as throbbing $(13 \%)$, lingering $(6 \%)$ or tingling $(3 \%)$. Both the average intensity and character of the pain did not differ significantly between ALA-PDT and MAL-PDT. In general, pain was experienced during illumination ( $84 \%$, first cycle) and only sporadically after illumination. Pain intensity was rated significantly higher during the second cycle in comparison to the first (4.27 vs. $3.47, p=0.011$ ), a difference mostly attributed to MAL (see Table 3.7).

In the first session, application of the cream was painful in twelve cases (38\%, all had undergone prior debulking), whereas in the second session, this was only reported once $(3 \%)$. Radiation of pain off the tumour area was not reported.

The commercial price of a two-gram packing of Metvix ${ }^{\oplus}$ in the Netherlands is currently 297 Euros. The all-in price of a two-gram packing as calculated by the pharmacist would, on approximation, correspond to 49.50 Euros. Inquiries from two other European university hospitals yielded prices of approximately 85 Euros for 5 grams and 80 Euros for 4 grams 
prepared 5-ALA cream (different base and different supplier of the raw material). The costs of the illumination device, dressing material, personnel costs and follow-up are equal for both precursors and were, therefore, not taken into consideration in this calculation.

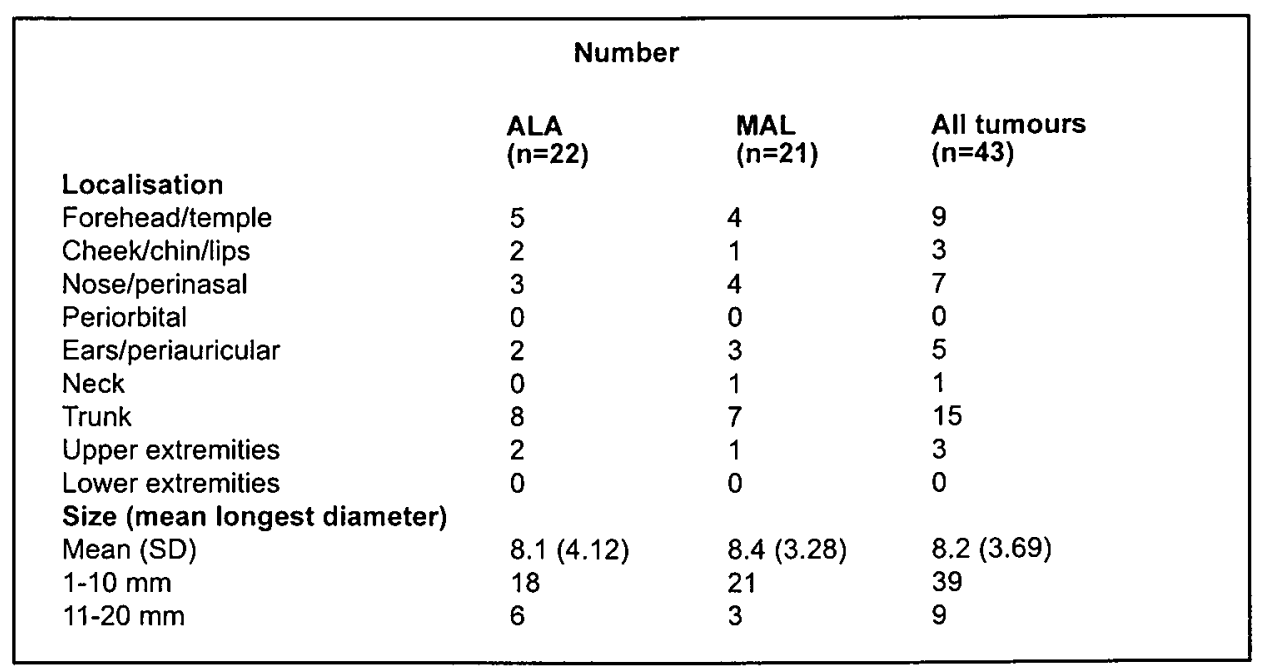

Table 3.5 Tumour characteristics

Incomplete clearance rates (\%)

$\begin{array}{lllll} & \begin{array}{l}\text { ALA } \\ (n=22)\end{array} & \begin{array}{l}\text { MAL } \\ (n=21)\end{array} & \begin{array}{l}\text { All tumours } \\ (\mathbf{n}=43)\end{array} & \text { P-value } \\ \text { Debulking } & 2 / 11(18.2) & 2 / 10(20.0) & 4 / 21(19.0) & \text { n.a. } \\ \text { No debulking } & 4 / 11(36.4) & 4 / 11(36.4) & 8 / 22(36.4) & \text { n.a. } \\ \text { Total } & 6 / 22(27.3) & 6 / 21(28.6) & 12 / 43(27.9) & 0.924 \\ \text { P-value } & \text { n.a. } & \text { n.a. } & 0.206 & \end{array}$

Table 3.6 Histologically incomplete clearance rates. 


\begin{tabular}{|lllll|}
\hline & \multicolumn{3}{c}{ VAS scores } & \\
& ALA & MAL & All tumours & $\begin{array}{l}\text { P-value } \\
\text { (MAL vs. ALA) }\end{array}$ \\
First treatment cycle & $(n=13)$ & $(n=19)$ & $(n=32)$ & \\
VAS score (SD) & $4.38(2.57)$ & $2.84(2.41)$ & $3.47(2.55)$ & 0.093 \\
Second treatment cycle & $(n=12)$ & $(n=18)$ & $(n=30)$ & \\
VAS score (SD) & $4.83(3.21)$ & $3.89(2.83)$ & $4.27(2.97)$ & 0.403 \\
$\begin{array}{l}\text { P-value } \\
\text { (first vs. second cycle) }\end{array}$ & 0.232 & 0.025 & 0.011 & \\
\end{tabular}

VAS=visual analogue scale, $A L A=$ deltaaminolevulinic acid, $M A L=$ methylester aminolevulinate

Table 3.7 Pain assessment

\section{Discussion}

These results show that there is no significant difference in short-term efficacy between topical ALA-PDT and MAL-PDT. Although the results are based on a small number of patients, histologically confirmed clearance rates around $72 \%$ were achieved for both ALAPDT and MAL-PDT in NBCC. Based on the literature and the results of this study it is unlikely that differences in efficacy will be apparent in larger numbers so that a powered randomised controlled trial therefore is not expected to be performed in the future.

The efficacy expressed in clearance rates obtained with histopathological control in this pilot study, are comparable to or even better than long-term results achieved from clinical follow-up, where incomplete response rates including initial non-responders range from $17 \%$ after one year up to $21 \%$ are achieved after three years follow-up. ${ }^{157.253} \mathrm{~A}$ discrepancy between initial clinical clearance and histological clearance has been described before. ${ }^{119}$ Randomised controlled studies with long-term follow-up comparing PDT to surgical excision are therefore essential and are currently being performed. ${ }^{157}$

Irrespective of the precursor used, residual tumour occurred more often in BCCs without prior debulking, although this was not statistically significant. However, this finding may have clinical relevance, and is in accordance with the literature, where clearance rates of $92 \%$ were achieced in topical ALA-PDT in $\mathrm{nBCC}$ with debulking three weeks prior to PDT, and $91 \%$ clearance rate after three months in the case of MAL. ${ }^{152,157}$ Possible explanations for the higher number of incomplete treatments reported here may be found in differences in illumination schemes or the timing and performance of the debulking. In cases where debulking took place immediately before the application of the cream, the cream often tended to lose contact with the skin surface due to protracted bleeding of the curetted surface, even after local haemostasis by pressure. The penetration of the precursor 
therefore cannot be guaranteed, which probably leads to a lower success rate in the first PDT cycle.

The pain experienced in the present study was comparable with rates described in the literature. ${ }^{263}$ Although the differences in pain between ALA and MAL-PDT did not reach statistical significance, there may be a clinically relevant trend. Patients treated with ALA evaluated their pain as slightly more intense than the patients treated with MAL, a finding which has been described before in healthy volunteers and in actinic keratosis. ${ }^{264.284}$ The underlying mechanism of pain caused by PDT has not been elucidated so far, but several theories have been proposed. Research has shown that 5-ALA, but not the ALA-ester, is transported into the cells via the system that normally transports $\gamma$-aminobuturic acid (GABA). ${ }^{277}$ GABA transporters are found in peripheral neurons, thus, ALA is expected to be taken up by peripheral nerve endings more than MAL. This might be an explanation for the difference in pain sensation. Patients rated the pain intensity as being higher during the second cycle compared to the first cycle. Possibly they were more anxious due to negative pain experiences in the first cycle. Also, the treatment areas still showed signs of inflammation and are therefore likely to be more painful when illuminated after one week. In the present trial, pain was often reported during application after debulking. This is well explainable since the cream is acidic to keep it stable and therefore likely to induce pain in open (curetted) wounds. ${ }^{285.286}$

Compared to the commercially available methyl ester-ALA (Metvix ${ }^{\circledR}$ ) 5-ALA cream is six times less expensive. The costs might even be halved if the illumination is not applied twice with an interval of one week as described in the protocol, but applied fractionated with an interval of several hours, as is suggested in (pre-)clinical studies. ${ }^{287,154}$ The cream is applied only once in such protocols. A disadvantage of the use of 5-ALA, however, is the necessity to prepare the cream freshly before use because there is still little known about its pharmacological stability. The advantage of Metvix ${ }^{\circledast}$ lies in the standardised preparation and protocol, its ease in use and the guaranteed availability and delivery. 


\section{Conclusion}

No statistically significant differences in the short-term efficacy and pain sensations between topical ALA-PDT and MAL-PDT were observed in this pilot study.

On the other hand, PDT with 5-ALA is less expensive than the commercially available methyl ester-ALA (Metvix $\left.{ }^{\circledast}\right)$.

It may, therefore, be concluded that both ALA and MAL may equally be recommended as photosensitisers in PDT for $\mathrm{nBCC}$ taking into account the fact that the efficacy of PDT for $\mathrm{nBCC}$ should be examined further in large randomised controlled trials with long-term clinical follow-up. 



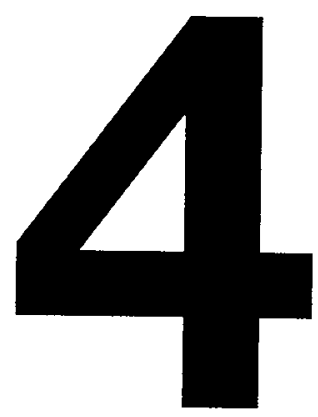

$\frac{O}{D}$
$\frac{D}{0}$
$\stackrel{\mathbb{D}}{D}$
$D$

Summary and general
discussion

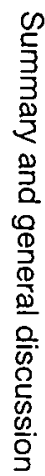




\section{Summary}

Basal cell carcinoma ( $\mathrm{BCC}$ ) is the most common skin malignancy with a strongly increasing incidence. The pathogenesis of $\mathrm{BCC}$ is complex with environmental, in which sunlight plays an important role, and genetic factors playing a crucial role. Different subtypes of $\mathrm{BCC}$ correspond to different growth patterns and different clinical appearances. Although mortality due to $B C C$ is very low, its high incidence and morbidity, leading to a high impact on health services, creates a need for early and effective treatment. Today, many treatment modalities for $\mathrm{BCC}$ exist, but relatively few randomised controlled trials (RCT) have been published. As a result, the decision-making for the treatment of a BCC is mainly based on clinical experience in addition to several tumour- and patient-related factors. The following aims were developed to contribute to a more evidence-based therapeutic choice. The results of the studies designed to answer these aims are summarised in this chapter.

\section{Is cryosurgery for non-aggressive type $\mathrm{BCC}$ as effective as surgical excision?}

Although cryosurgery (CS) is a common therapeutic strategy for BCC, so far the efficacy has never been compared to surgical excision (SE) in a randomised manner. CS was compared to SE in the treatment of mainly nodular BCC in a randomised trial with five years' follow-up. The overall five-year recurrence probability was $19.6 \%$ for CS and $8.4 \%$ for SE. Although no statistically significant difference between the techniques was evident due to underpowered numbers, in the opinion of the authors the large differences observed are of clinical relevance. Besides this, SE has several advantages over CS, as is observed in previous studies, such as a better cosmetic result, a shorte wound care period and histopathological control. Based on these facts combined with the high recurrence percentage after CS, we believe that SE should be preferred to $C S$ in the treatment of primary, non-aggressive $\mathrm{BCC}$ of the head and neck and that the place for CS in BCC should be reserved for patients unable or unwilling to undergo surgery. The difficulty in drawing conclusions from underpowered studies underlines the importance of an adequately performed study design including a power analysis.

\section{Is Mohs' micrographic surgery an effective treatment for BCC?}

Mohs' micrographic surgery (MMS) is an advanced excision technique with intra-operative histopathological examination, which is often used to treat BCC in the USA. It is practised on a smaller scale in Europe. The effectiveness of MMS in the treatment of 720 facial BCCs was evaluated retrospectively in the authors' dermatology department. The five-year recurrence rates (Kaplan-Meier) estimated from this study were $3.2 \%$ for primary $\mathrm{BCC}$ and $6.7 \%$ for recurrent $\mathrm{BCC}$. Prognostic factors for recurrence were an ag- 
gressive histopathologic subtype, more than four Mohs' stages, a large defect size and a recurrent BCC. Based on these low recurrence rates compared to other treatment modalities, the authors consider MMS as the treatment of first choice for primary facial $B C C$ s with an aggressive histopathologic subtype and in recurrent BCCs in the face.

\section{May Mohs' micrographic surgery be combined with other, non-invasive, treatment modalities in patients with large, ill-defined BCC with combined subtype?}

Many BCCs show a combined histopathological subtype, with a partially aggressive often central part and a partially non-aggressive, often periferal part. ${ }^{52}$ Occasionally, Mohs' procedures have to be discontinued prematurely for unforeseen reasons. As the authors are convinced of the need for further treatment after incomplete treatment, possibilities for the adjuvant treatment of patients in which superficial BCC remained after incomplete Mohs' surgery were investigated. Seven cases of patients are reported where MMS for their large BCCs was discontnued for different unforeseen reasons with remaining superficial fields of BCC after the aggressive and deeply localised part had been removed. Three weeks after closure of the defect, adjuvant treatment for the superficial remnants of the tumour with imiquimod $5 \%$ cream or with PDT was started. The treatments were well accepted with good cosmetic results and no recurrences after a follow-up period ranging from 13 to 30 months. This series of cases suggests a place for less invasive treatment modalities such as imiquimod $5 \%$ cream as well as for PDT as adjuvant therapy for remnants of superficial BCC after multiple stages of MMS for mixed-type BCC in patients with extensive sun damage, multiple facial BCCs and extensive surgery in the past or for those cases in which MMS has to be terminated prematurely for unforeseen reasons. Accurate follow-up studies are necessary to determine the efficacy in the long-term.

\section{Does systemic azithromycin improve the survival of full thickness grafts on the nose?}

The most common localisation of BCC for which surgery is performed is the nose. Defects are often closed with full thickness grafts (FTG), which sometimes heal with partial or entire necrosis. In the absence of a proven efficient protocol for the use of systemic antibiotics in cases of FTG on the nose, a RCT was performed in which the use of azithromycin was compared to standard treatment in 30 patients who underwent a FTG reconstruction of a surgical defect on the nose after surgery of non-melanoma skin cancer. A statistically significant difference in favour of the grafts treated with azithromycin was found. Smoking was the only variable of those analysed that had a significant negative effect on the survival of the graft. We therefore advocate the use of systemic antibiotics with accurate bacterial spectrum such as azithromycin, in FTG reconstruction after surgery of 
non-melanoma skin cancer of the nose. Smoking should be strongly discouraged. Future randomised trials are necessary to investigate whether topical antibiotics are as effective as systemic antibiotics.

\section{Is photodynamic therapy for $\mathrm{nBCC}$ equally effective as surgical excision?}

PDT has proven to be an effective treatment for SBCC, but for $n B C C$ the long-term efficacy has not yet been established. An RCT was carried out which included a total of 175 primary $n B C C s$ in 150 patients. Tumours were randomly assigned to either PDT $(n=84)$ or SE $(n=91)$. Fractionated ALA-PDT was performed with two illuminations with equal fluence rates. The results of an interim analysis that has been carried out are described in this thesis. Incomplete responses or recurrences were observed in 17 BCCs, 16 times after PDT and once after SE. The overall 36 months-disease free probability based on the Kaplan-Meier survival analysis was $66.5 \%$ for PDT and $98.8 \%$ for $S E(p<0.001)$.

Although final results can only be drawn from the results after five years, the present results with the relatively high percentage of incomplete treatments and recurrences would appear to make PDT less suitable as a regular treatment for nBCC.

\section{Are photodynamic therapy performed with methylaminole- vulinate and PDT performed with aminolevulinate equally effective with regard to efficacy and pain?}

Two topical photosensitisers, 5-aminolevulininate (ALA) and methyl aminolevulinate (MAL) are widely used at present in dermatology. The degree of pain and the costs of ALA-PDT versus MAL-PDT were evaluated in 43 nodular BCCs (nBCC). Residual tumour tissue was detected in $28 \%$ of the cases. No difference between ALA-PDT and MAL-PDT was found with regard to therapeutic efficacy and pain scores. However, the costs of MAL-PDT were six times higher than those of ALA-PDT. It is, therefore, concluded that both ALA and MAL may be applied equally as topical photosensitisers in PDT for BCC.

\section{How painful is topical PDT in $\mathrm{nBCC}$ ?}

Pain is regarded as a major side effect of topical PDT, particularly when treating large areas of non-melanoma skin cancer. This pain can even be a limiting factor in the treatment of BCC. Understanding the mechanism of pain in PDT will lead to solutions for the better management of this side effect and will result in an expansion of the practical application of the therapy. Further studies are therefore needed to unravel the mechanisms of pain in PDT. The quality and intensity of pain was assessed in $64 \mathrm{nBCC}$ s treated with ALA (ALAPDT), using a visual analogue scale (VAS). The average pain intensity was 3.88 , with most patients experiencing burning $(82.5 \%)$ or stinging $(36.8 \%)$ sensations. 
Treatment of single BCCs with ALA-PDT rarely resulted in unbearable pain and was never a reason to discontinue the treatment. Illumination with the Medeikonos ${ }^{*}$ light source was experienced as being less painful than with the Waldmann lamp. It will be valuable to carry out a comparison with newer light sources based on light emitting diodes (LED)-lights in the future as these lamps are increasingly being used in dermatological practices.

\section{General discussion}

Nowadays, there are many ways to treat a patient with one or more BCCs. The decision to use a particular therapy is based on both tumour characteristics and patient-related aspects. These factors, however, are not treatment-specific and often several treatments with equal effectiveness are available and applicable.

Therefore, it is almost impossible to design a flow chart for the treatment of BCC which guides the clinician through the decision-making process.

There is a great need for effective treatments that are easy to perform due to the rising incidence of BCC. Dermatologists should have knowledge about the treatment modalities available for $\mathrm{BCC}$ and these should be a part of their training.

SE and CS are still the most frequently applied treatments for BCC. Surgical intervention is the golden standard because this technique is the only one offering the possibility of carrying out a histopathological check of resection margins.

MMS is probably the most effective treatment but it is not necessary that this be performed on all BCCs because of the investment of time and higher costs. Smaller and less aggressive tumours can also be treated by standard SE. There is no standard alternative in cases where MMS is not available or not needed, but more treatment modalities can then be taken into consideration.

CS can be applied quickly for a low price. The disadvantages of CS, however, are the relatively higher risk of recurrence and unfavourable scars. Treatment margins can be enlarged to minimise the recurrence risk, but wider margins in CS will certainly lead to unacceptable cosmetic results. In cases where patients cannot be operated upon and the cosmetic result is of lesser importance, CS is a good alternative. Because cartilage is unsusceptible to freezing, CS is also an appropriate treatment for smaller, non-aggressive BCCs on the eyelids and ears.

As the literature of RT in BCC is mainly based on former and already abandoned protocols, there is a need for new studies, preferably RCTs, to determine the present role for $\mathrm{RT}$ in the treatment of BCC. 
Stimulation of the immune system leading to selective tumour regression is a promising future development for the treatment of relatively recent tumours.

In stead of taking wider margins to achieve complete tumour response, and thus increasing the risk for undesired scars, the implementation of the new, highly tumour selective treatment modalities of imiquimod and PDT is a good alternative. The mechanism of action of these therapies is aimed particularly at malignant cells. The healthy surrounding tissue is saved and this will obviously be in favour of the final cosmetic result.

All treatments have advantages as well as disadvantages, and imiquimod and PDT are not applicable for the use with all types of BCC because of the limiting effectiveness in deep and aggressive BCC. Long-term efficacy studies are still running, but the first results are promising for superficial growing tumours. Results of PDT seem less favourable for the nodular tumours. The application of PDT and imiquimod may be considered particularly for patients with multiple superficial BCCs.

With the majority of tumours being small and not aggressive together with the rising incidence in young patients with a longer life expectancy, there is a danger that the sub-optimal treatment of patients in the long-term may lead to severe invasive, recurrent tumours.

In this light, there is a growing need for techniques to visualise tumour cells in the depth, in order to delineate tumour invasion. With a larger number of BCCs consisting of both aggressive and non-aggressive tumour parts, such techniques would aid the clinician in taking the decision to apply a particular treatment.

With the development of new treatment modalities and a continuous stream of new study results becoming available, the treatment of patients with BCC can sometimes deviate from existing guidelines. Combinations of treatment modalities for one patient or for one tumour should be considered in this era where the treatment of patients with BCC is becoming more and more customised. 


\section{Bibliography}

1

2

3

Jacob A. Observations respecting an ulcer of peculiar character, which attacks the eyelids and other parts of the face. Dublin Hospital Rep Commun Med Surg 1827; 4: 232-9.

Rigel DS, Friedman RJ, Kopf AW. Lifetime risk for development of skin cancer in the U.S. population: current estimate is now 1 in 5. J Am Acad Dermatol 1996; 35: 1012-3.

de Vries $E$, Louwman $M$, Bastiaens $M$ et al. Rapid and continuous increases in incidence rates of basal cell carcinoma in the southeast Netherlands since 1973. J Invest Dermatol 2004; 123: 634-8.

de Vries E, van de Poll-Franse LV, Louwman WJ et al. Predictions of skin cancer incidence in the Netherlands up to 2015. Br J Dermatol 2005; 152: 481-8.

Thissen MR, Neumann HA, Berretty PJ et al. [The treatment of basal cell carcinoma patients by dermatologists in the Netherlands]. Ned Tijdschr Geneeskd 1998; 142: 1563-7.

Christenson LJ, Borrowman TA, Vachon CM et al. Incidence of basal cell and squamous cell carcinomas in a population younger than 40 years. Jama 2005; 294: 681-90.

de Vries $E$, Coebergh JW, van der Rhee $H$. [Trends, causes, approach and consequences related to the skin-cancer epidemic in the Netherlands and Europe]. Ned Tijdschr Geneeskd 2006; 150: 1108-15.

Staples MP, Elwood M, Burton RC et al. Non-melanoma skin cancer in Australia: the 2002 national survey and trends since 1985. Med J Aust 2006; 184: 6-10.

Zanetti R, Rosso S, Martinez C et al. The multicentre south European study 'Helios'. I: Skin characteristics and sunburns in basal cell and squamous cell carcinomas of the skin. $\mathrm{Br} \mathrm{J}$ Cancer 1996; 73: 1440-6.

Goldberg LH. Basal cell carcinoma. Lancet 1996; 347: 663-7.

Alexander GA, Henschke UK. Advanced skin cancer in Tanzanian albinos: preliminary observations. J Nat/ Med Assoc 1981; 73: 1047-54.

Lang PG, Maize JC. Basal Cell Carcinoma. In: Cancer of the skin (Rigel D, ed), Vol. 1: Elsevier, 2005: 101-32.

Wei Q, Matanoski GM, Farmer ER et al. DNA repair and aging in basal cell carcinoma: a molecular epidemiology study. Proc Natl Acad Sci U S A 1993; 90: 1614-8.

Kricker A, Armstrong BK, English DR et al. Does intermittent sun exposure cause basal cell carcinoma? A case-control study in Western Australia. Int J Cancer 1995; 60: 489-94.

Zanetti $R$, Rosso S, Martinez $C$ et al. Comparison of risk patterns in carcinoma and melanoma of the skin in men: a multi-centre case-case-control study. Br J Cancer 2006; 94: 743-51.

Karagas MR, Stannard VA, Mott LA et al. Use of tanning devices and risk of basal cell and squamous cell skin cancers. J Natl Cancer Inst 2002; 94: 224-6.

Naldi $L$, DiLandro A, D'Avanzo B et al. Host-related and environmental risk factors for cutaneous basal cell carcinoma: evidence from an Italian case-control study. J Am Acad Dermatol 2000; 42: 446-52.

Gallagher RP, Hill GB, Bajdik CD et al. Sunlight exposure, pigmentary factors, and risk of nonmelanocytic skin cancer. I. Basal cell carcinoma. Arch Dermatol 1995; 131: 157-63.

Strickland FM, Kripke ML. Immune response associated with nonmelanoma skin cancer. Clin Plast Surg 1997; 24: 637-47.

Auepemkiate $S$, Boonyaphiphat $P$, Thongsuksai $P$. $p 53$ expression related to the aggressive infiltrative histopathological feature of basal cell carcinoma. Histopathology 2002; 40: 56873.

Krekels G, Voorter C, Kuik F et al. DNA-protection by sunscreens: Using p53-immunostaining. Eur J Dermatol 1997; 7: 259-62.

Tilli CM, Van Steensel MA, Krekels GA et al. Molecular aetiology and pathogenesis of basal cell carcinoma. Br J Dermatol 2005; 152: 1108-24.

Verhaegh ME, Sanders CJ, Arends JW et al. Expression of the apoptosis-suppressing protein Bcl-2 in non-melanoma skin cancer. Br J Dermatol 1995; 132: 740-4.

Wikonkal NM, Berg RJ, van Haselen CW et al. bcl-2 vs p53 protein expression and apoptotic rate in human nonmelanoma skin cancers. Arch Dermatol 1997; 133: 599-602.

Moloney FJ, Comber H, O'Lorcain $\mathrm{P}$ et al. A population-based study of skin cancer incidence and prevalence in renal transplant recipients. Br J Dermatol 2006; 154: 498-504.

Bavinck JN, Bastiaens MT, Marugg ME et al. Further evidence for an association of HLA-DR7 with basal cell carcinoma on the tropical island of Saba. Arch Dermatol 2000; 136: 1019-22. 

Infect Dis 2002; 15: 101-14.

28 Karagas MR, Nelson $\mathrm{HH}$, Sehr $\mathrm{P}$ et al. Human papillomavirus infection and incidence of squamous cell and basal cell carcinomas of the skin. J Natl Cancer Inst 2006; 98: 389-95. Badri T, Zeglaoui F, Kochbati L et al. [Multiple basal cell carcinomas following radiation therapy for nasopharyngeal cancer]. Presse Med 2006; 35: 55-7. Leisenring W, Friedman DL, Flowers ME et al. Nonmelanoma skin and mucosal cancers after hematopoietic cell transplantation. J Clin Oncol 2006; 24: 1119-26.

31 Centeno JA, Mullick FG, Martinez L et al. Pathology related to chronic arsenic exposure. Environ Health Perspect 2002; 110 Suppl 5: 883-6.

32 Marmelzat WL. Malignant tumors in smallpox vaccination scars: a report of 24 cases. Arch Dermatol 1968; 97: 400-6.

33 Cribier B, Scrivener Y, Grosshans E. Tumors arising in nevus sebaceus: A study of 596 cases. $J$ Am Acad Dermatol 2000; 42: 263-8.

34 Novick M, Gard DA, Hardy SB et al. Burn scar carcinoma: a review and analysis of 46 cases. J Trauma 1977; 17: 809-17.

35 Ozyazgan I. Kontas O. Previous injuries or scars as risk factors for the development of basal cell carcinoma. Scand J Plast Reconstr Surg Hand Surg 2004; 38: 11-5.

36 Telfer NR, Colver GB, Bowers PW. Guidelines for the management of basal cell carcinoma. British Association of Dermatologists. Br J Dermatol 1999; 141: 415-23.

37 Lee DA, Grossman ME, Schneiderman $P$ et al. Genetics of skin appendage neoplasms and related syndromes. J Med Genet 2005; 42: 811-9.

38 Gailani MR, Stahle-Backdahl M, Leffell DJ et al. The role of the human homologue of Drosophila patched in sporadic basal cell carcinomas. Nat Genet 1996; 14: 78-81.

39 Lam CW, Xie J, To KF et al. A frequent activated smoothened mutation in sporadic basal cell carcinomas. Oncogene 1999; 18: 833-6.

40 Bodak N, Queille S, Avril MF et al. High levels of patched gene mutations in basal-cell carcinomas from patients with xeroderma pigmentosum. Proc Natl Acad SciU S A 1999; 96: 511722.

41 Asada M, Schaart FM, de Almeida HL, Jr. et al. Solid basal cell epithelioma (BCE) possibly originates from the outer root sheath of the hair follicle. Acta Derm Venereol 1993; 73: 28692.

42 Kumakiri M, Hashimoto K. Ultrastructural resemblance of basal cell epithelioma to primary epithelial germ. J Cutan Pathol 1978; 5: 53-67.

43 Reidbord HE, Wechsler HL, Fisher ER. Ultrastructural study of basal cell carcinoma and its variants with comments on histogenesis. Arch Dermatol 1971; 104: 132-40. Zackheim HS. Origin of the human basal cell epithelioma. J Invest Dermatol 1963; 40: 28397.

Asplund A, Sivertsson A, Backvall $H$ et al. Genetic mosaicism in basal cell carcinoma. Exp Dermatol 2005; 14: 593-600.

46 Motley RJ, Gould DJ, Douglas WS et al. Treatment of basal cell carcinoma by dermatologists in the United Kingdom. British Association of Dermatologists Audit Subcommittee and the British Society for Dermatological Surgery. Br J Dermatol 1995; 132: 437-40.

Wade TR, Ackerman AB. The many faces of basal-cell carcinoma. J Dermatol Surg Oncol 1978; 4: 23-8.

48 Rippey JJ. Why classify basal cell carcinomas? Histopathology 1998; 32: 393-8.

49 Emmett AJ. Surgical analysis and biological behaviour of 2277 basal cell carcinomas. Aust $N$ Z J Surg 1990; 60: 855-63.

50 Raasch BA, Buettner PG, Garbe C. Basal cell carcinoma: histological classification and bodysite distribution. Br J Dermatol 2006; 155: 401-7.

51 Smeets $\mathrm{N}$. Refining indications for Mohs micrographic surgery in treatment of facial basal cell carcinoma. In: Dermatology, Vol. PhD. Maastricht: University of Maastricht, 2004: 1-138.

Cohen PR, Schulze KE, Nelson BR. Basal cell carcinoma with mixed histology: a possible pathogenesis for recurrent skin cancer. Dermatol Surg 2006; 32: 542-51.

53 Kaur P, Mulvaney M, Andrew Carlson J. Basal Cell Carcinoma Progression Correlates With Host Immune Response and Stromal Alterations: A Histologic Analysis. Am J Dermatopathol 2006; 28: 293-307.

54 Leffell DJ, Headington JT, Wong DS et al. Aggressive-growth basal cell carcinoma in young adults. Arch Dermatol 1991; 127: 1663-7. 
Kopf AW. Computer analysis of 3531 basal-cell carcinomas of the skin. J Dermatol 1979; 6 : 267-81.

Bastiaens MT, Hoefnagel JJ, Bruijn JA et al. Differences in age, site distribution, and sex between nodular and superficial basal cell carcinoma indicate different types of tumors. J Invest Dermatol 1998; 110: 880-4.

McCormack CJ, Kelly JW, Dorevitch AP. Differences in age and body site distribution of the histological subtypes of basal cell carcinoma. A possible indicator of differing causes. Arch Dermatol 1997; 133: 593-6.

Swanson NA. Mohs surgery. Technique, indications, applications, and the future. Arch Dermatol 1983; 119: 761-73.

59 Mohs FE, Lathrop TG. Modes of spread of cancer of skin. AMA Arch Derm Syphilol 1952; 66: 427-39.

Mark GJ. Basal cell carcinoma with intraneural invasion. Cancer 1977; 40: 2181-7.

Ratner D, Lowe L, Johnson TM et al. Perineural spread of basal cell carcinomas treated with Mohs micrographic surgery. Cancer 2000; 88: 1605-13.

Hendrix JD, Jr., Parlette HL. Micronodular basal cell carcinoma. A deceptive histologic subtype with frequent clinically undetected tumor extension. Arch Dermatol 1996; 132: 295-8. tensions in a series of 51 cases. J Dermatol Surg Oncol 1981; 7: 387-94.

Germann G, Bernstein-Sommer B, Petrovici V et al. [Differential, oncologically adequate therapy of basalioma]. Handchir Mikrochir Plast Chir 1992; 24: 151-8.

Miller PK, Roenigk RK, Brodland DG et al. Cutaneous micrographic surgery: Mohs procedure. Mayo Clin Proc 1992; 67: 971-80.

Snow SN, Sahl W, Lo JS et al. Metastatic basal cell carcinoma. Report of five cases. Cancer 1994; 73: 328-35.

Sahl WJ, Jr., Snow SN, Levine NS. Giant basal cell carcinoma. Report of two cases and review of the literature. J Am Acad Dermatol 1994; 30: 856-9.

69 Lang PG, Jr., Maize JC. Histologic evolution of recurrent basal cell carcinoma and treatment implications. J Am Acad Dermatol 1986; 14: 186-96.

Reymann F. Treatment of basal cell carcinoma of the skin with 5-fluorouracil ointment. A 10year follow-up study. Dermatologica 1979; 158: 368-72.

71 Yarosh $\mathrm{D}$, Klein $\mathrm{J}, \mathrm{O}^{\prime}$ Connor $\mathrm{A}$ et al. Effect of topically applied T4 endonuclease $\mathrm{V}$ in liposomes on skin cancer in xeroderma pigmentosum: a randomised study. Xeroderma Pigmentosum Study Group. Lancet 2001; 357: 926-9.

72 Tilli CM, Stavast-Kooy AJ, Vuerstaek JD et al. The garlic-derived organosulfur component ajoene decreases basal cell carcinoma tumor size by inducing apoptosis. Arch Dermatol Res 2003; 295: 117-23.

73 Buechner SA. Intralesional interferon alfa-2b in the treatment of basal cell carcinoma. Immunohistochemical study on cellular immune reaction leading to tumor regression. J Am Acad Dermatol 1991; 24: 731-4.

74 Beutner KR, Geisse JK, Helman D et al. Therapeutic response of basal cell carcinoma to the immune response modifier imiquimod 5\% cream. J Am Acad Dermatol 1999; 41: 1002-7.

75 Levine $N$. Role of retinoids in skin cancer treatment and prevention. J Am Acad Dermatol 1998; 39: S62-6.

76 Taipale J, Chen JK, Cooper MK et al. Effects of oncogenic mutations in Smoothened and Patched can be reversed by cyclopamine. Nature 2000; 406: 1005-9.

77 Bianchi L, Orlandi A, Campione $E$ et al. Topical treatment of basal cell carcinoma with tazarotene: a clinicopathological study on a large series of cases. Br J Dermatol 2004; 151: 14856.

78 Campolmi P, Mavilia L, Bonan $\mathrm{P}$ et al. $595 \mathrm{~nm}$ pulsed dye laser for the treatment of superficial basal cell carcinoma. Lasers Med Sci 2005; 20: 147-8.

79 Smeets NW, Kuijpers DI, Nelemans $P$ et al. Mohs' micrographic surgery for treatment of basal cell carcinoma of the face--results of a retrospective study and review of the literature. $\mathrm{Br} J$ Dermatol 2004; 151: 141-7.

80 Lang PG. Variables to consider in the management of non-melanoma skin cancer. $J$ Geriatr Dermatol 1996; 4: 231-7.

81 Breuninger $\mathrm{H}$, Dietz K. Prediction of subclinical tumor infiltration in basal cell carcinoma. $J$ Dermatol Surg Oncol 1991; 17: 574-8. 
Breuninger $H$, Rassner $G$, Undeutsch $W$. [Surgical treatment of basaliomas with calculated safety margin and control of the histologic borders. Experiences with 355 tumors]. Hautarzt 1984; 35: 303-7.

83 Wolf DJ, Zitelli JA. Surgical margins for basal cell carcinoma. Arch Dermatol 1987; 123: 340 4.

84 Beljaards R, Neumann HAM. Oncological dermatosurgery, University of Maastricht, 1996; chapter 11:135-44.

Neumann HAM. Approach to the oncological patient. In: Oncological dermatosurgery. (RC Beljaards, ed). Maastricht, the Netherlands: University of Maastricht, 1996: Chapter 11,135-46. Kooy AJ, Tank B, de Jong TA et al. Expression of cytokeratin 8 in basal cell carcinoma: a comparative immunohistochemical and immunoelectron microscopy study. Anticancer Res 1996; 16: $277-82$.

87 Thissen $M$, Neumann $H$. The sense and non-sense of immunohistochemical staining in basal cell carcinomas and other cutaneous tumors. Skin Cancer 1997; 12: 37-42.

Abide JM, Nahai F, Bennett RG. The meaning of surgical margins. Plast Reconstr Surg 1984; 73: 492-7.

89 Dellon AL, DeSilva $S$, Connolly $M$ et al. Prediction of recurrence in incompletely excised basal cell carcinoma. Plast Reconstr Surg 1985; 75: 860-71.

90 Richmond JD, Davie RM. The significance of incomplete excision in patients with basal cell carcinoma. Br J Plast Surg 1987; 40: 63-7.

91 Robinson JK, Fisher SG. Recurrent basal cell carcinoma after incomplete resection. Arch Dermatol 2000; 136: 1318-24.

92 Thissen M. Treatment of basal cell carcinoma in the light of photodynamic therapy (Thesis). Maastricht: University of Maastricht, 2000.

93 Krekels G, Verhaegh M, Neumann H. Mohs' micrografische chirurgie. Ned Tijdschr Dermatol Venereol 1996; 6: 90-3.

94 Silverman MK, Kopf AW, Bart RS et al. Recurrence rates of treated basal cell carcinomas. Part 3: Surgical excision. J Dermatol Surg Oncol 1992; 18: 471-6.

95 Thissen MR, Neumann MH, Schouten LJ. A systematic review of treatment modalities for primary basal cell carcinomas. Arch Dermatol 1999; 135: 1177-83.

96 Rowe DE, Carroll RJ, Day CL, Jr. Long-term recurrence rates in previously untreated (primary) basal cell carcinoma: implications for patient follow-up. J Dermatol Surg Oncol 1989; 15: 315-28.

97 Sakura CY, Calamel PM. Comparison of treatment modalities for recurrent basal cell carcinoma. Plast Reconstr Surg 1979; 63: 492-6.

98 Smeets N, Neumann M. Mohs' micrographic surgery for basal-cell carcinoma of the face. Lancet 2005; 365: 1227.

99 Bauer M, Loosli RM, Anderl $\mathrm{H}$ et al. [Surgical treatment of malignant skin epitheliomas. Principles, methods, results]. Chirurg 1977; 48: 170-9.

100 Smeets NW, Krekels GA, Ostertag JU et al. Surgical excision vs Mohs' micrographic surgery for basal-cell carcinoma of the face: randomised controlled trial. Lancet 2004; 364: 1766-72.

101 Avril MF, Auperin A, Margulis A et al. Basal cell carcinoma of the face: surgery or radiotherapy? Results of a randomized study. Br J Cancer 1997; 76: 100-6.

102 Thissen MR, Nieman FH, Ideler AH et al. Cosmetic results of cryosurgery versus surgical excision for primary uncomplicated basal cell carcinomas of the head and neck. Dermatol Surg 2000; 26: 759-64.

103 Mohs FE. Chemosurgery: microscopically controlled surgery for skin cancer--past, present and future. J Dermatol Surg Oncol 1978; 4: 41-54.

104 Julian CG, Bowers PW. A prospective study of Mohs' micrographic surgery in two English centres. Br J Dermatol 1997; 136: 515-8.

105 Rowe DE, Carroll RJ, Day CL, Jr. Mohs surgery is the treatment of choice for recurrent (previously treated) basal cell carcinoma. J Dermatol Surg Oncol 1989; 15: 424-31.

106 Wennberg AM, Larko O, Stenquist B. Five-year results of Mohs' micrographic surgery for aggressive facial basal cell carcinoma in Sweden. Acta Derm Venereol 1999; 79: 370-2.

107 Mohs F, Larson P, Iriondo M. Micrographic surgery for the microscopically controlled excision of carcinoma of the external ear. J Am Acad Dermatol 1988; 19: 729-37.

108 Mohs FE. Micrographic surgery for the microscopically controlled excision of eyelid cancer: history and development. Adv Ophthalmic Plast Reconstr Surg 1986; 5: 381-408. 

698-703.

110 Essers BA, Dirksen CD, Nieman FH et al. Cost-effectiveness of Mohs Micrographic Surgery vs Surgical Excision for Basal Cell Carcinoma of the Face. Arch Dermatol 2006; 142: 18794.

111 Kopke LF, Konz B. [Micrographic surgery. A current methodological assessment]. Hautarzt 1995; 46: 607-14.

112 Allington HV. Liquid nitrogen in the treatment of skin diseases. Calif Med 1950; 72: 153.

113 Zacarian SA. Cryosurgery for skin cancer. In: Zacarian SA, ed. Cryosurgery for skin cancer and cutaneous disorders. St.Louis: CV Mosby 1985; 97: 96-162.

114 Fraunfelder FT, Zacarian SA, Wingfield DL et al. Results of cryotherapy for eyelid malignancies. Am J Ophthalmol 1984; 97: 184-8.

115 Torre D. Cryosurgical instrumentation and depth dose monitoring. Clin Dermatol 1990; 8: 4860.

116 Nordin P, Larko O, Stenquist B. Five-year results of curettage-cryosurgery of selected large primary basal cell carcinomas on the nose: an alternative treatment in a geographical area underserved by Mohs' surgery. Br J Dermatol 1997; 136: 180-3.

117 Lindgren $\mathrm{G}$, Larko $O$. Long-term follow-up of cryosurgery of basal cell carcinoma of the eyelid. J Am Acad Dermatol 1997; 36: 742-6.

118 Anders $\mathrm{M}$, Sporl E, Krantz $\mathrm{H}$ et al. [Cryotherapy of malignant eyelid tumors]. Ophthalmologe 1995; 92: 787-92.

119 Wang I, Bendsoe N, Klinteberg CA et al. Photodynamic therapy vs. cryosurgery of basal cell carcinomas: results of a phase III clinical trial. Br J Dermatol 2001; 144: 832-40.

120 Mallon E, Dawber R. Cryosurgery in the treatment of basal cell carcinoma. Assessment of one and two freeze-thaw cycle schedules. Dermatol Surg 1996; 22: 854-8.

121 Hall VL, Leppard BJ, McGill J et al. Treatment of basal-cell carcinoma: comparison of radiotherapy and cryotherapy. Clin Radiol 1986; 37: 33-4.

122 Kuflik EG. Cryosurgery for skin cancer: 30-year experience and cure rates. Dermatol Surg $2004 ; 30: 297-300$.

123 Thissen $M$. Treatment of basal cell carcinoma in the light of photodynamic therapy (Thesis). Maastricht:Maastricht University ISBN 90-5681-073-1, 2000: 38.

124 Kopf AW, Bart RS, Schrager D et al. Curettage-electrodesiccation treatment of basal cell carcinomas. Arch Dermatol 1977; 113: 439-43.

125 Werlinger KD, Upton G, Moore AY. Recurrence rates of primary nonmelanoma skin cancers treated by surgical excision compared to electrodesiccation-curettage in a private dermatological practice. Dermatol Surg 2002; 28: 1138-42; discussion 42.

126 Scholten AN, Griep C, Davelaar $\mathrm{J}$ et al. [Electron beam irradiation is effective in the treatment of skin carcinomas; a comparison with superficial roentgen therapy]. Ned Tijdschr Geneeskd 1996; 140: 428-31.

127 Olschewski T, Bajor K, Lang B et al. [Radiotherapy of basal cell carcinoma of the face and head: Importance of low dose per fraction on long-term outcome]. J Dtsch Dermatol Ges 2006; 4: 124-30.

128 Silverman MK, Kopf AW, Gladstein $\mathrm{AH}$ et al. Recurrence rates of treated basal cell carcinomas. Part 4: X-ray therapy. J Dermatol Surg Oncol 1992; 18: 549-54.

129 Zagrodnik B, Kempf W, Seifert B et al. Superficial radiotherapy for patients with basal cell carcinoma: recurrence rates, histologic subtypes, and expression of p53 and Bcl-2. Cancer 2003; 98: 2708-14.

130 Caccialanza M, Piccinno $R$, Beretta $M$ et al. Results and side effects of dermatologic radiotherapy: a retrospective study of irradiated cutaneous epithelial neoplasms. J Am Acad Dermatol 1999; 41: 589-94.

131 Reymann F, Kopp H. Treatment of basal cell carcinoma of the skin with ultrasoft X-rays. Dermatologica 1978; 156: 40-7.

132 Schneiter M, Krebs A. [Late therapeutic, functional and cosmetic results in 103 patients treated with soft radiotherapy for 117 cases of basaloma]. Dermatologica 1982; 165: 342-51.

133 Holmes ME, Bomford CK. The use of a short distance cobalt unit in the treatment of primary skin tumours. Br J Radiol 1982; 55: 225-8.

134 Smith SP, Grande DJ. Basal cell carcinoma recurring after radiotherapy: a unique, difficult treatment subclass of recurrent basal cell carcinoma. J Dermatol Surg Oncol 1991; 17: 2630 . 
Davis MM, Hanke CW, Zollinger TW et al. Skin cancer in patients with chronic radiation dermatitis. J Am Acad Dermatol 1989; 20: 608-16.

136 Beswick SJ, Garrido MC, Fryer AA et al. Multiple basal cell carcinomas and malignant melanoma following radiotherapy for ankylosing spondylitis. Clin Exp Dermatol 2000; 25: 381-3.

137 Brown SB, Brown EA, Walker I. The present and future role of photodynamic therapy in cancer treatment. Lancet Oncol 2004; 5: 497-508.

138 Nowis D, Makowski M, Stoklosa T et al. Direct tumor damage mechanisms of photodynamic therapy. Acta Biochim Pol 2005; 52: 339-52.

139 van Duijnhoven FH, Aalbers RI, Rovers JP et al. The immunological consequences of photodynamic treatment of cancer, a literature review. Immunobiology 2003; 207: 105-13.

140 Almeida RD, Manadas BJ, Carvalho AP et al. Intracellular signaling mechanisms in photodynamic therapy. Biochim Biophys Acta 2004; 1704: 59-86.

141 Oleinick NL, Morris RL, Belichenko I. The role of apoptosis in response to photodynamic therapy: what, where, why, and how. Photochem Photobiol Sci 2002; 1: 1-21.

142 Fritsch C, Goerz G, Ruzicka T. Photodynamic therapy in dermatology. Arch Dermatol 1998; 134: 207-14.

143 de Blois AW, Thissen MR, de Bruijn HS et al. In vivo pharmacokinetics of protoporphyrin IX accumulation following intracutaneous injection of 5 -aminolevulinic acid. $J$ Photochem Photobiol B 2001; 61: 21-9.

144 Casas $A$, Fukuda $H$, Meiss $R$ et al. Topical and intratumoral photodynamic therapy with 5aminolevulinic acid in a subcutaneous murine mammary adenocarcinoma. Cancer Lett 1999; 141: 29-38.

145 Gerscher S, Connelly JP, Beijersbergen Van Henegouwen GM et al. A quantitative assessment of protoporphyrin IX metabolism and phototoxicity in human skin following dose-controlled delivery of the prodrugs 5-aminolaevulinic acid and 5-aminolaevulinic acid-n-pentylester. Br J Dermatol 2001; 144: 983-90.

146 Gaullier JM, Berg K, Peng $Q$ et al. Use of 5-aminolevulinic acid esters to improve photodynamic therapy on cells in culture. Cancer Res 1997; 57: 1481-6.

147 Fritsch $C$, Homey B, Stahl $W$ et al. Preferential relative porphyrin enrichment in solar keratoses upon topical application of delta-aminolevulinic acid methylester. Photochem Photobiol 1998; 68: 218-21.

148 Morton CA, Mackie RM, Whitehurst $C$ et al. Photodynamic therapy for basal cell carcinoma: effect of tumor thickness and duration of photosensitizer application on response. Arch Dermatol 1998; 134: 248-9.

149 Szeimies RM, Sassy T, Landthaler M. Penetration potency of topical applied delta-aminolevulinic acid for photodynamic therapy of basal cell carcinoma. Photochem Photobiol 1994; 59 : 73-6.

150 Svanberg $\mathrm{K}$, Andersson T, Killander $\mathrm{D}$ et al. Photodynamic therapy of non-melanoma malignant tumours of the skin using topical delta-amino levulinic acid sensitization and laser irradiation. Br J Dermatol 1994; 130: 743-51.

151 Thissen MR, de Blois MW, Robinson DJ et al. PplX fluorescence kinetics and increased skin damage after intracutaneous injection of 5-aminolevulinic acid and repeated illumination. $J$ Invest Dermatol 2002; 118: 239-45.

152 Thissen MR, Schroeter CA, Neumann HA. Photodynamic therapy with delta-aminolaevulinic acid for nodular basal cell carcinomas using a prior debulking technique. $\mathrm{Br} J$ Dermatol 2000 ; 142: $338-9$.

153 Soler AM, Warloe T, Tausjo J et al. Photodynamic therapy by topical aminolevulinic acid, dimethylsulphoxide and curettage in nodular basal cell carcinoma: a one-year follow-up study. Acta Derm Venereol 1999; 79: 204-6.

154 de Haas ER, Kruijt B, Sterenborg $\mathrm{HJ}$ et al. Fractionated Illumination Significantly Improves the Response of Superficial Basal Cell Carcinoma to Aminolevulinic Acid Photodynamic Therapy. $J$ Invest Dermatol 2006; 126(12): 2679-86.

155 Wennberg AM, Lindholm LE, Alpsten M et al. Treatment of superficial basal cell carcinomas using topically applied delta-aminolaevulinic acid and a filtered xenon lamp. Arch Dermatol Res 1996; 288: 561-4.

156 Fink-Puches R, Soyer HP, Hofer A et al. Long-term follow-up and histological changes of superficial nonmelanoma skin cancers treated with topical delta-aminolevulinic acid photodynamic therapy. Arch Dermatol 1998; 134: 821-6. 
157 Rhodes LE, de Rie $M$, Enstrom $Y$ et al. Photodynamic therapy using topical methyl aminolevulinate vs surgery for nodular basal cell carcinoma: results of a multicenter randomized prospective trial. Arch Dermatol 2004; 140: 17-23.

158 Wennberg AM, Gudmundson $F$, Stenquist $B$ et al. In vivo detection of basal cell carcinoma using imaging spectroscopy. Acta Derm Venereol 1999; 79: 54-61.

159 Fritsch C, Neumann NJ, Ruzicka T et al. [Photodiagnostic tests. 3: Fluorescence diagnosis with delta-aminolevulinic acid-induced porphyrins (FDAP) in dermatology]. Hautarzt 2000; 51: 528-43; quizz 43-5.

160 Arany I, Tyring SK, Stanley MA et al. Enhancement of the innate and cellular immune response in patients with genital warts treated with topical imiquimod cream $5 \%$. Antiviral Res 1999; 43: 55-63.

161 Marini M. Imiquimod 5\% cream: a topical immune response modifier. Int J Dermatol 2002; 41 Suppl 1: 1-2.

162 Stanley MA. Imiquimod and the imidazoquinolones: mechanism of action and therapeutic potential. Clin Exp Dermatol 2002; 27: 571-7.

163 Urosevic $M$, Maier T, Benninghoff $B$ et al. Mechanisms underlying imiquimod-induced regression of basal cell carcinoma in vivo. Arch Dermatol 2003; 139: 1325-32.

164 Dummer R, Urosevic $M$, Kempf $W$ et al. Imiquimod in basal cell carcinoma: how does it work? Br J Dermatol 2003; 149 Suppl 66: 57-8.

165 Berman B, Sullivan T, De Araujo T et al. Expression of Fas-receptor on basal cell carcinomas after treatment with imiquimod 5\% cream or vehicle. Br J Dermatol 2003; 149 Suppl 66: 5961.

166 Wenzel J, Uerlich M, Haller $O$ et al. Enhanced type I interferon signaling and recruitment of chemokine receptor $\mathrm{CXCR} 3$-expressing lymphocytes into the skin following treatment with the TLR7-agonist imiquimod. J Cutan Pathol 2005; 32: 257-62.

167 Geisse J, Caro I, Lindholm J et al. Imiquimod 5\% cream for the treatment of superficial basal cell carcinoma: results from two phase III, randomized, vehicle-controlled studies. J Am Acad Dermatol 2004; 50: 722-33.

168 Geisse JK, Rich P, Pandya A et al. Imiquimod 5\% cream for the treatment of superficial basal cell carcinoma: a double-blind, randomized, vehicle-controlled study. J Am Acad Dermatol 2002; 47: 390-8.

169 Gollnick H, Barona CG, Frank RG et al. Recurrence rate of superficial basal cell carcinoma following successful treatment with imiquimod $5 \%$ cream: interim 2-year results from an ongoing five-year follow-up study in Europe. Eur J Dermatol 2005; 15: 374-81.

170 Gebauer K, Quirk C, De'Ambrosis B. Imiquimod 5\% cream for treatment of superficial basal cell carcinoma: a long-term follow-up study in Australia. J Eur Acad Dermatol Venereol 2003; 17(Suppl.3): 203.

171 Shumack S, Robinson J, Kossard S et al. Efficacy of topical $5 \%$ imiquimod cream for the treatment of nodular basal cell carcinoma: comparison of dosing regimens. Arch Dermatol 2002; 138: $1165-71$.

172 Sterry W, Ruzicka T, Herrera E et al. Imiquimod $5 \%$ cream for the treatment of superficial and nodular basal cell carcinoma: randomized studies comparing low-frequency dosing with and without occlusion. Br J Dermatol 2002; 147: 1227-36.

173 Huber A, Huber JD, Skinner RB, Jr. et al. Topical imiquimod treatment for nodular basal cell carcinomas: an open-label series. Dermatol Surg 2004; 30: 429-30.

174 Vidal D, Matias-Guiu X, Alomar A. Open study of the efficacy and mechanism of action of topical imiquimod in basal cell carcinoma. Clin Exp Dermatol 2004; 29: 518-25.

175 Thissen MR, Kuijpers DI, Krekels GA. Local immune modulator (imiquimod $5 \%$ cream) as adjuvant treatment after incomplete Mohs micrographic surgery for large, mixed type basal cell carcinoma: a report of 3 cases. $J$ Drugs Dermatol 2006; 5 : 461-4.

176 Torres A, Niemeyer A, Berkes B et al. 5\% imiquimod cream and reflectance-mode confocal microscopy as adjunct modalities to Mohs micrographic surgery for treatment of basal cell carcinoma. Dermatol Surg 2004; 30: 1462-9.

177 Sapijaszko MJ. Imiquimod 5\% cream (Aldara) in the treatment of basal cell carcinoma. Skin Therapy Lett 2005; 10: 2-5.

178 Stenquist B, Wennberg AM, Gisslen $\mathrm{H}$ et al. Treatment of aggressive basal cell carcinoma with intralesional interferon: evaluation of efficacy by Mohs surgery. J Am Acad Dermatol 1992; $27: 65-9$. 
179 Cornell RC, Greenway HT, Tucker SB et al. Intralesional interferon therapy for basal cell carcinoma. J Am Acad Dermatol 1990; 23: 694-700.

180 Guthrie TH, Jr., McElveen LJ, Porubsky ES et al. Cisplatin and doxorubicin. An effective chemotherapy combination in the treatment of advanced basal cell and squamous carcinoma of the skin. Cancer 1985; 55: 1629-32.

181 Humphreys TR, Malhotra R, Scharf MJ et al. Treatment of superficial basal cell carcinoma and squamous cell carcinoma in situ with a high-energy pulsed carbon dioxide laser. Arch Dermatol 1998; 134: 1247-52.

182 Brenner S, Wolf R, Dascalu DI. Topical tretinoin treatment in basal cell carcinoma. J Dermatol Surg Oncol 1993; 19: 264-6.

183 Kuflik EG, Gage AA, Lubritz RR et al. Millenium paper: history of dermatologic cryosurgery. Dermatol Surg 2000; 26: 715-22.

184 Kokoszka A, Scheinfeld N. Evidence-based review of the use of cryosurgery in treatment of basal cell carcinoma. Dermatol Surg 2003; 29: 566-71.

185 Nordin P, Stenquist B. Five-year results of curettage-cryosurgery for 100 consecutive auricular non-melanoma skin cancers. J Laryngol Otol 2002; 116: 893-8.

186 Buschmann W. A reappraisal of cryosurgery for eyelid basal cell carcinomas. Br J Ophthalmol 2002; 86: 453-7.

187 Clark TG, Bradburn MJ, Love SB et al. Survival analysis part I: basic concepts and first analyses. Br J Cancer 2003; 89: 232-8.

188 Bradburn MJ, Clark TG, Love SB et al. Survival analysis part II: multivariate data analysis--an introduction to concepts and methods. Br J Cancer 2003; 89: 431-6.

189 Bath-Hextall $F$, Bong J, Perkins $W$ et al. Interventions for basal cell carcinoma of the skin: systematic review. Bmj 2004; 329: 705.

190 Bogdanov-Berezovsky A, Cohen AD, Glesinger $R$ et al. Risk factors for incomplete excision of basal cell carcinomas. Acta Derm Venereol 2004; 84: 44-7.

191 Bumpous JM, Padhya TA, Barnett SN. Basal cell carcinoma of the head and neck: identification of predictors of recurrence. Ear Nose Throat J 2000; 79: 200-2, 4.

192 Rigel DS, Robins P, Friedman RJ. Predicting recurrence of basal-cell carcinomas treated by microscopically controlled excision: a recurrence index score. J Dermatol Surg Oncol 1981; 7: 807-10.

193 Hohmann DH, Milewski C, Krieger $U$ et al. [Esthetic aspects of excision of basaliomas of the area of the head and neck]. Laryngorhinootologie 1992; 71: 311-4.

194 Marghoob AA. Basal and squamous cell carcinomas. What every primary care physician should know. Postgrad Med 1997; 102: 139-42, 46, 52-4 passim.

195 Holme SA, Malinovszky K, Roberts DL. Changing trends in non-melanoma skin cancer in South Wales, 1988-98. Br J Dermatol 2000; 143: 1224-9.

196 Kuijpers DI, Thissen MR, Neumann MH. Basal cell carcinoma: treatment options and prognosis, a scientific approach to a common malignancy. Am J Clin Dermatol 2002; 3: 247-59.

197 Bower CP, Lear JT, de Berker DA. Basal cell carcinoma follow-up practices by dermatologists: a national survey. Br J Dermato/ 2001; 145: 949-56.

198 Mohs F. Chemosurgery: a microscopically controlled method of cancer excision. Archives of surgery 1941; 42: 279-95.

199 Shriner DL, McCoy DK, Goldberg DJ et al. Mohs micrographic surgery. J Am Acad Dermatol 1998; 39: 79-97.

200 Gaston DA, Naugle C, Clark DP. Mohs micrographic surgery referral patterns: the University of Missouri experience. Dermatol Surg 1999; 25: 862-6.

201 Neumann HAM, Krekels GAM, Verheagh MEJM. Treatment of 208 extensive basal cell carcinomas with Mohs micrographic surgery. J Eur Acad Dermatol Venereol 1996; 6: 217-25.

202 Mohs FE, Zitelli JA. Microscopically controlled surgery in the treatment of carcinoma of the scalp. Arch Dermatol 1981; 117: 764-9.

203 Mohs FE. Micrographic surgery for the microscopically controlled excision of eyelid cancers. Arch Ophthalmol 1986; 104: 901-9.

204 Tromovitch TA, Beirne G, Beirne C. Mohs' technique (cancer chemosurgery). Treatment of recurrent cutaneous carcinomas. Cancer 1966; 19: 867-8.

205 Robins $\mathrm{P}$, Rodriguez-Sains $\mathrm{R}$, Rabinovitz $\mathrm{H}$ et al. Mohs surgery for periocular basal cell carcinomas. J Dermatol Surg Oncol 1985; 11: 1203-7.

206 Silverman MK, Kopf AW, Grin CM et al. Recurrence rates of treated basal cell carcinomas. Part 1: Overview. J Dermatol Surg Oncol 1991; 17: 713-8. 
207 Marcil I, Stern RS. Risk of developing a subsequent nonmelanoma skin cancer in patients with a history of nonmelanoma skin cancer: a critical review of the literature and meta-analysis. Arch Dermatol 2000; 136: 1524-30.

208 Welch ML, Anderson LL, Grabski WJ. Evaluation and management of nonmelanoma skin cancer. The military perspective. Dermatol Clin 1999; 17: 19-28, vii.

209 Rodriguez-Sains RS, Robins P, Smith B et al. Radiotherapy of periocular basal cell carcinomas: recurrence rates and treatment with special attention to the medical canthus. $\mathrm{Br} J$ Ophthalmol 1988; 72: 134-8.

210 Silverman MK, Kopf AW, Grin CM et al. Recurrence rates of treated basal cell carcinomas. Part 2: Curettage-electrodesiccation. J Dermatol Surg Oncol 1991; 17: 720-6.

211 Eliezri YD, Cohen PR. Cancer recurrence following Mohs micrographic surgery: a mechanism of tumor persistence. Plast Reconstr Surg 1992; 90: 121-5.

212 Dzubow LM. False-negative tumor-free margins following Mohs surgery. J Dermatol Surg Oncol 1988; 14: 600-2.

213 Smeets NW, Stavast-Kooy AJ, Krekels GA et al. Adjuvant cytokeratin staining in Mohs micrographic surgery for basal cell carcinoma. Dermatol Surg 2003; 29: 375-7.

214 Hruza GJ. Mohs micrographic surgery local recurrences. J Dermatol Surg Oncol 1994; 20: 573-7.

215 Lang PG, Jr., McKelvey AC, Nicholson JH. Three-dimensional reconstruction of the superficial multicentric basal cell carcinoma using serial sections and a computer. Am J Dermatopathol 1987; 9: 198-203.

216 Madani S, Huilgol SC, Carruthers A. Unplanned incomplete Mohs micrographic surgery. J Am Acad Dermatol 2000; 42: 814-9.

217 Testerman TL, Gerster JF, Imbertson LM et al. Cytokine induction by the immunomodulators imiquimod and S-27609. J Leukoc Biol 1995; 58: 365-72.

218 Sidky YA, Borden EC. Weeks CE et al. Inhibition of murine tumor growth by an interferon-inducing imidazoquinolinamine. Cancer Res 1992; 52: 3528-33.

219 Saldanha G, Fletcher A, Slater DN. Basal cell carcinoma: a dermatopathological and molecular biological update. Br J Dermatol 2003; 148: 195-202.

220 Sexton M, Jones DB, Maloney ME. Histologic pattern analysis of basal cell carcinoma. Study of a series of 1039 consecutive neoplasms. J Am Acad Dermatol 1990; 23: 1118-26.

221 Grabski WJ, Salasche SJ. Positive surgical excision margins of a basal cell carcinoma. Dermatol Surg 1998; 24: 921-4.

222 Nagore E, Grau C, Molinero J et al. Positive margins in basal cell carcinoma: relationship to clinical features and recurrence risk. A retrospective study of 248 patients. $J$ Eur Acad Dermatol Venereol 2003; 17: 167-70.

223 Friedman HI, Williams $\mathrm{T}$, Zamora $\mathrm{S}$ et al. Recurrent basal cell carcinoma in margin-positive tumors. Ann Plast Surg 1997; 38: 232-5.

224 Sussman LA, Liggins DF. Incompletely excised basal cell carcinoma: a management dilemma? Aust N Z J Surg 1996; 66: 276-8.

225 Kuijpers DI, Smeets NW, Krekels GA et al. Photodynamic therapy as adjuvant treatment of extensive basal cell carcinoma treated with Mohs micrographic surgery. Dermatol Surg 2004; 30: 794-8.

226 Marks R, Gebauer K, Shumack S et al. Imiquimod 5\% cream in the treatment of superficial basal cell carcinoma: results of a multicenter 6-week dose-response trial. J Am Acad Dermatol 2001; 44: 807-13.

227 Morton CA, Brown SB, Collins S et al. Guidelines for topical photodynamic therapy: report of a workshop of the British Photodermatology Group. Br J Dermatol 2002; 146: 552-67.

228 Pariser DM, Lowe JL, Stewart DM et al. Photodynamic therapy with topical methyl aminolevulinate for actinic keratosis:results of a prospective randomized multicenter trial. J Am Acad Dermatol 2003; 48: 227-32.

229 Morton CA, Whitehurst C, McColl JH et al. Photodynamic therapy for large or multiple patches of Bowen disease and basal cell carcinoma. Arch Dermatol 2001; 137: 319-24.

230 Peng $Q$, Warloe T, Berg $K$ et al. 5-Aminolevulinic acid-based photodynamic therapy. Clinical research and future challenges. Cancer 1997; 79: 2282-308.

231 Robinson JK, Dillig $\mathrm{G}$. The advantages of delayed nasal full-thickness skin grafting after Mohs micrographic surgery. Dermatol Surg 2002; 28: 845-51.

232 Thibault MJ, Bennett RG. Success of delayed full-thickness skin grafts after Mohs micrographic surgery. J Am Acad Dermatol 1995; 32: 1004-9. 
Maragh SL, Otley CC, Roenigk RK et al. Antibiotic prophylaxis in dermatologic surgery: updated guidelines. Dermatol Surg 2005; 31: 83-91.

234 Sebben JE. Prophylactic antibiotics in cutaneous surgery. J Dermato/ Surg Oncol 1985; 11: 901-6.

235 Perl TM, Cullen JJ, Wenzel RP et al. Intranasal mupirocin to prevent postoperative Staphylococcus aureus infections. N Engl J Med 2002; 346: 1871-7.

236 Scheinfeld NS, Tutrone WD, Torres $O$ et al. Macrolides in dermatology. Clin Dermatol 2003; 21: 40-9.

237 Jennings MB, McCarty JM, Scheffler NM et al. Comparison of azithromycin and cefadroxil for the treatment of uncomplicated skin and skin structure infections. Cutis 2003; 72: 240-4.

238 Alvarez-Elcoro S, Enzler MJ. The macrolides: erythromycin, clarithromycin, and azithromycin. Mayo Clin Proc 1999; 74: 613-34.

239 Parsad D, Pandhi R, Dogra S. A guide to selection and appropriate use of macrolides in skin infections. Am J Clin Dermatol 2003; 4: 389-97.

240 Amaya-Tapia G, Aguirre-Avalos $G$, Andrade-Villanueva $J$ et al. Once-daily azithromycin in the treatment of adult skin and skin-structure infections. J Antimicrob Chemother 1993; 31 Suppl E: 129-35.

241 Huether MJ, Griego RD, Brodiand DG et al. Clindamycin for intraincisional antibiotic prophylaxis in dermatologic surgery. Arch Dermatol 2002; 138: 1145-8.

242 Silverstein P. Smoking and wound healing. Am J Med 1992; 93: 22S-4S.

243 Wong LS, Martins-Green M. Firsthand cigarette smoke alters fibroblast migration and survival: implications for impaired healing. Wound Repair Regen 2004; 12: 471-84.

244 Sorensen LT, Karlsmark T, Gottrup F. Abstinence from smoking reduces incisional wound infection: a randomized controlled trial. Ann Surg 2003; 238: 1-5.

245 Hubbard TJ. Leave the fat, skip the bolster: thinking outside the box in lower third nasal reconstruction. Plast Reconstr Surg 2004; 114: 1427-35.

246 Goldminz D, Bennett RG. Cigarette smoking and flap and full-thickness graft necrosis. Arch Dermatol 1991; 127: 1012-5.

247 Silapunt S, Peterson SR, Alam M et al. Clinical appearance of full-thickness skin grafts of the nose. Dermatol Surg 2005; 31: 177-83.

248 Babilas $P$, Karrer S, Sidoroff A et al. Photodynamic therapy in dermatology--an update. Photodermatol Photoimmunol Photomed 2005; 21: 142-9.

249 Soler AM, Warloe T, Berner A et al. A follow-up study of recurrence and cosmesis in completely responding superficial and nodular basal cell carcinomas treated with methyl 5-aminolaevulinate-based photodynamic therapy alone and with prior curettage. $\mathrm{Br} J$ Dermatol 2001; 145 : 467-71.

250 Horn $M$, Wolf $P$, Wulf $\mathrm{HC}$ et al. Topical methyl aminolaevulinate photodynamic therapy in patients with basal cell carcinoma prone to complications and poor cosmetic outcome with conventional treatment. Br J Dermatol 2003; 149: 1242-9.

251 Calzavara-Pinton PG. Repetitive photodynamic therapy with topical delta-aminolaevulinic acid as an appropriate approach to the routine treatment of superficial non-melanoma skin tumours. J Photochem Photobiol B 1995; 29: 53-7.

252 Van Oosten EJ, Kuijpers DIM, Thissen MRTM. Different pain sensations in photodynamic therapy for nodular basal cell carcinoma. Photodiagnosis and Photodynamic Therapy 2006; 3: $61-8$.

253 Rhodes LE, De Rie M, Enstrom $Y$ et al. A randomized European comparison of excision surgery and MAL-PDT in nodular basal cell carcinoma: results from a 36 month follow up. abstract 14th Congress of the European Academy of Dermatology and Venereology. 2005; 12-16 October.

254 Kuijpers DIM, Thissen MRT, Thissen CAC et al. Similar effectiveness of methyl aminolevulinate and 5-aminolevulinate in topical photodynamic therapy for Nodular Basal cell carcinoma. J Drugs Dermatol 2006; 5: 642-5.

255 Avril MF, Auperin A, Margulis A et al. Basal cell carcinoma of the face: surgery or radiotherapy? Results of a randomized study. Br J Cancer 1997; 76: 100-6.

256 Zeitouni NC, Oseroff AR, Shieh S. Photodynamic therapy for nonmelanoma skin cancers. Current review and update. Mol Immunol 2003; 39: 1133-6.

257 Marmur ES, Schmults CD, Goldberg DJ. A review of laser and photodynamic therapy for the treatment of nonmelanoma skin cancer. Dermatol Surg 2004; 30: 264-71. 
Beier C, Kaufmann R. Photodynamic therapy as complementary treatment option for facial basal cell carcinoma. In: The 10th World Congress on Cancers of the Skin. Vienna, 2005.

Peng Q, Moan J, Nesland JM. Correlation of subcellular and intratumoral photosensitizer localization with ultrastructural features after photodynamic therapy. Ultrastruct Pathol 1996; 20: $109-29$.

260 Juzeniene $A$, Juzenas $P, M a L W$ et al. Effectiveness of different light sources for 5-aminolevulinic acid photodynamic therapy. Lasers in Medical Science 2004; 19: 139-49.

261 Touma D, Yaar M, Whitehead $S$ et al. A trial of short incubation, broad-area photodynamic therapy for facial actinic keratoses and diffuse photodamage. Arch Dermatol 2004; 140: 3340.

262 Beljaards RC, S P. Fotodynamische therapie met methylaminolevulinaat: een leidraad voor uitvoering van de behandeling. Nederlands Tijdschrift voor Dermatologie \& Venereologie 2004; 14: 299-306.

263 Grapengiesser S, Ericson M, Gudmundsson F et al. Pain caused by photodynamic therapy of skin cancer. Clin Exp Dermatol 2002; 27: 493-7.

264 Wiegell $S R$, Stender $1 M, N a R$ et al. Pain associated with photodynamic therapy using 5aminolevulinic acid or 5-aminolevulinic acid methylester on tape-stripped normal skin. Arch Dermatol 2003; 139: 1173-7.

265 Basset-Seguin N IS, Emtestam L et al. Photodynamic therapy using Metvix is as efficacious as cryotherapy in BCC, with better cosmetic results. J Eur Acad Dermatol Venereol 2001; 15 Sup.2: 226.

266 Varma S, Wilson H, Kurwa HA et al. Bowen's disease, solar keratoses and superficial basal cell carcinomas treated by photodynamic therapy using a large-field incoherent light source. Br J Dermatol 2001; 144: 567-74.

267 Dragieva G, Prinz BM, Hafner J et al. A randomized controlled clinical trial of topical photodynamic therapy with methyl aminolaevulinate in the treatment of actinic keratoses in transplant recipients. Br J Dermatol 2004; 151: 196-200.

268 Morton CA, Burden AD. Treatment of multiple scalp basal cell carcinomas by photodynamic therapy. Clin Exp Dermatol 2001; 26: 33-6.

269 Nielsen $K$, Juzeniene $A$, Juzenas $P$ et al. Choice of optimal wavelengt for PDT: The significance of oxygen depletion. Photochem Photobiol 2005; Apr 1.

270 Morton CA. Photodynamic therapy for nonmelanoma skin cancer-and more? Arch Dermatol 2004; 140: 116-20.

271 Fritsch $C$, Stege $H$, Saalmann $G$ et al. Green light is effective and less painful than red light in photodynamic therapy of facial solar keratoses. Photodermatol Photoimmunol Photomed 1997; 13: 181-5.

272 Holmes MV, Dawe RS, Ferguson J et al. A randomized, double-blind, placebo-controlled study of the efficacy of tetracaine gel (Ametop) for pain relief during topical photodynamic thrapy. $\mathrm{Br}$ $J$ Dermotol 2004; 150 : 337-40.

273 Pagliaro J, Elliott T, Bulsara $M$ et al. Cold air analgesia in photodynamic therapy of basal cell carcinomas and bowen's disease: An effective addition to treatment: A pilot study. Dermatol Surg 2004; 30: 63-6.

274 Monfrecola G, Procaccini EM, D'Onofrio D et al. Hyperpigmentation induced by topical 5-aminolaevulinic acid plus visible light. $J$ Photochem Photobiol B 2002; 68: 147-55.

275 Scott J, Huskisson EC. Graphic representation of pain. Pain 1976; 2: 175-84.

276 Orenstein A, Kostenich G, Tsur $\mathrm{H}$ et al. Temperature monitoring during photodynamic therapy of skin tumors with topical 5-aminolevulinic acid application. Cancer Lett 1995; 93: 227-32.

277 Rud E, Gederaas O, Hogset A et al. 5-aminolevulinic acid, but not 5-aminolevulinic acid esters, is transported into adenocarcinoma cells by system BETA transporters. Photochem Photobiol 2000; 71: 640-7.

278 Ericson MB, Sandberg C, Stenquist B et al. Photodynamic therapy of actinic keratosis at varying fluence rates: assessment of photobleaching, pain and primary clinical outcome. $\mathrm{Br} J$ Dermatol 2004; 151: 1204-12.

279 Dijkstra AT, Majoie IM, van Dongen JW et al. Photodynamic therapy with violet light and topical 6-aminolaevulinic acid in the treatment of actinic keratosis, Bowen's disease and basal cell carcinoma. J Eur Acad Dermatol Venereol 2001; 15: 550-4.

280 Casas A, Batlle A. Rational design of 5-aminolevulinic acid derivatives aimed at improving photodynamic therapy. Curr Med Chem Anti-Canc Agents 2002; 2: 465-75. 
281 He D, Sassa S, Lim HW. Effect of UVA and blue light on porphyrin biosynthesis in epidermal cells. Photochem Photobiol 1993; 57 : 825-9.

282 Basset-Seguin N BY, Pavel S et al. A dose finding study of photodynamic therapy with Metvix in patients with basal cell carcinoma (abstract). J Eur Acad Dermatol Venereol 2000; 14 Suppl. 1: 39.

283 Foley P. Methyl aminolaevulinate photodynamic therapy in practice: Treatment protocol. Australas J Dermatol 2005; 45: S14.

284 Kasche A, Luderschmidt S, Ring J et al. Photodynamic therapy induces less pain in patients treated with methyl aminolevulinate compared to aminolevulinic acid. J Drugs Dermatol 2006; 5: 353-6.

285 de Blois AW, Grouls RJ, Ackerman EW et al. Development of a stable solution of 5-aminolaevulinic acid for intracutaneous injection in photodynamic therapy. Lasers Med Sci 2002; 17: 208-15.

286 Fukuda $\mathrm{H}$, Casas A, Batlle A. Aminolevulinic acid: from its unique biological function to its star role in photodynamic therapy. Int J Biochem Cell Biol 2005; 37: 272-6.

287 Robinson DJ, de Bruijn HS, Star WM et al. Dose and timing of the first light fraction in two-fold illumination schemes for topical ALA-mediated photodynamic therapy of hairless mouse skin. Photochem Photobiol 2003; 77: 319-23. 


\section{Samenvatting}

Het basaalcelcarcinoom (BCC) is de meest voorkomende vorm van huidkanker met een sterk stijgende incidentie. De pathogenese van BCC is complex waarin omgevingsfactoren (o.a. zonlicht) en patiëntgebonden factoren een belangrijke rol spelen. Er zijn verschillende klinische subtypen van het BCC bekend die corresponderen met verschillende histopathologische groeiwijzen.

Ondanks dat de mortaliteit van BCC laag is, zijn de morbiditeit en de kosten voor de gezondheidszorg bij deze tumor hoog, waardoor het belang van een snelle en effectieve behandeling groot is. Ondanks dat er vandaag de dag een breed scala aan behandelingen (met deels overlappende indicaties) voor het BCC bestaat, zijn er relatief weinig gerandomiseerde, gecontroleerde studies over de behandeling van het $\mathrm{BCC}$ gepubliceerd. Hierdoor wordt de keuze voor behandeling van een patiënt met BCC voornamelijk gebaseerd op klinische ervaring van de behandelend arts, en worden tumor- en patiëntgerelateerde factoren niet altijd ten volle in de overweging betrokken.

Om bij te dragen aan een meer 'evidence-based' besluitvorming, zijn de volgende vraagstellingen van dit proefschrift geformuleerd. In dit hoofdstuk worden de resultaten van de studies die uitgevoerd zijn om deze vraagstellingen te beantwoorden, samengevat.

Is cryochirurgie voor niet-agressieve BCCs even effectief als chirurgische excisie? Cryochirurgie (CS) is een veel gebruikte behandeling voor het $\mathrm{BCC}$ die snel en goedkoop kan worden toegepast. De effectiviteit is echter nog niet eerder gerandomiseerd vergeleken met de standaardbehandeling, chirurgische excisie (SE). In een gerandomiseerde, gecontroleerde opzet met vijf jaar follow-up werd CS vergeleken met SE in de behandeling van voornamelijk nodulaire, het meest voorkomende subtype, BCCs (nBCCs). Het vijfjaars recidiefpercentage berekend middels Kaplan-Meier survivalanalyse bedroeg $19.6 \%$ voor CS en $8.4 \%$ voor SE. Dit verschil bereikte geen statistische significantie bereikte $(p=0.10)$, waarschijnlijk omwille van een te kleine onderzoekspopulatie. Desondanks heeft dit verschil in recidiefpercentage ons inziens wel klinische relevantie. Andere voordelen van SE, zoals gebleken in andere studies, zijn de histologische controle achteraf, het betere cosmetisch resultaat t.o.v. CS en de kortere duur van wondgenezing. Op basis van deze gegevens en het hoge recidiefpercentage na $C S$, verdient in onze opinie SE de voorkeur boven $\mathrm{CS}$ in de behandeling van niet-agressieve BCC in de hoofd-hals regio en dient CS gereserveerd te worden voor patiënten die niet geopereerd kunnen of willen worden.

Is Mohs' micrografische chirurgie een effectieve behandeling voor BCC?

Mohs' micrografische chirurgie (MMS) is een geavanceerde operatietechniek met perop- 
eratief, histopathologisch onderzoek. Deze techniek wordt op grote schaal toegepast bij BCCs in de Verenigde Staten. In Europa wordt het op minder grote schaal toegepast in de behandeling van $\mathrm{BCC}$. Wij onderzochten retrospectief de effectiviteit van MMS in 720 BCCs die op onze afdeling zijn uitgevoerd in de periode april 1992 tot december 1999. Vijf-jaars recidiefpercentages berekend middels Kaplan-Meier survivalanalyse bedroegen $3.2 \%$ voor primaire $\mathrm{BCCs}$ en $6.7 \%$ voor recidief $\mathrm{BCCs}$. Prognostische factoren voor een recidief $B C C$ zijn agressief groeiende $B C C s$, meerdere $(\geq 3)$ rondes, grote ( $>40 \mathrm{~mm}$ diameter) defecten en recidief tumoren. Gebaseerd op deze lage recidiefpercentages vergeleken met andere behandelingen voor BCC, kan MMS beschouwd worden als behandeling van eerste keus voor primaire, agressieve $\mathrm{BBCs}$ in het gelaat en voor recidief BCCs in het gelaat.

Zou Mohs' micrografische chirurgie gecombineerd kunnen worden met andere, niet invasieve behandelingsmodaliteiten bij patiënten met grote, slecht afgrensbare BCC van gecombineerd subtype?

Een groot deel van de BCCs is opgebouwd uit meerdere histopathologische subtypes. Een veel voorkomende combinatie is een BCC met een agressief centraal deel, waarvoor MMS geïndiceerd is en een niet agressief, superficiëel groeiend deel aan de periferie. Met name dit perifere gedeelte kan tegenwoordig ook met niet-invasieve technieken zoals imiquimod en fotodynamische therapie (PDT) worden behandeld waardoor het onnodig opofferen van huid voorkomen kan worden. Mohs' procedures moeten soms voortijdig beëindigd worden om onvoorziene redenen.

Zeven patiënten worden besproken, waarbij de Mohs' procedure om verschillende redenen werd afgebroken en de patiënt drie weken na het sluiten van het defect werd nabehandeld met $5 \%$ imiquimod crème in drie gevallen of met PDT in vier gevallen. De behandelingen werden goed verdragen en er werden geen recidieven waargenomen na een follow-up periode variërend van 13 tot 30 maanden. Deze patiëntenreeks suggereert een plaats voor PDT en imiquimod als adjuvante therapie voor restanten van superficiëel BCC na meerdere rondes van MMS bij patiënten met uitgebreide zonbeschadigde huid, multipele of grote BCCs in het gelaat of bij patiënten bij wie de Mohs' procedure omwille van onvoorziene redenen voortijdig moet worden beëindigd. Studies met lange follow-up en grotere patiëntenaantallen zijn nodig om de effectiviteit van deze combinatietherapie op langere termijn vast te stellen.

Verbetert systemische azithromycine de overleving van volledige-dikte huidtransplantaten op de neus?

De meest voorkomende lokalisatie van BCC in het gelaat is de neus. Defecten na chirurgische behandelingen kunnen op verschillende manieren gesloten worden. Een daarvan 
is het sluiten d.m.v. volle-dikte transplantaten (FTG). De genezing van een wond bedekt met FTG gaat vaak gepaard met gedeeltelijke of gehele necrose. Er is nog geen bewijs dat het gebruik van antibiotica deze necrotisering kan voorkomen en een algemene richtlijn voor het gebruik van systemische antibiotica bij FTG op de neus ontbreekt tot op heden. Wij voerden een gerandomiseerd, gecontroleerd onderzoek uit naar het effect van azithromycine op de overleving van de FTG bij 30 patiënten bij wie een defect op de neus na chirurgische behandeling van niet-melanoom huidkanker werd gesloten met een FTG. Omwille van praktische redenen werd gekozen voor orale toediening van de antibiotica een maal daags gedurende 3 dagen aansluitend aan de ingreep. Een statistisch significant verschil ten gunste van het gebruik van azithromycine werd gevonden. Van alle variabelen die onderzocht zijn, had roken een significant negatief effect op de overleving van het transplantaat. Op basis van deze resultaten kan het gebruik van azithromycine worden geadviseerd bij patiënten met een FTG op de neus na chirurgische behandeling van nietmelanoom huidkanker. In de toekomst zouden studies kunnen worden uitgevoerd om te onderzoeken of lokale antibiotica voor deze indicatie even effectief zijn als systemische antibiotica.

\section{Is fotodynamische therapie voor nodulair BCC even effectief als chirurgische excisie?} PDT heeft in eerdere studies bewezen een effectieve behandeling te zijn voor superficiëel $\mathrm{BCC}(\mathrm{sBCC})$. Of PDT voor nodulaire BCC ( $\mathrm{nBCC}$ ) op lange termijn een effectieve behandeling is, is nog niet vastgesteld. Een gerandomiseerde, gecontroleerde studie met 175 primaire BCCs bij 150 patiënten werd uitgevoerd. BCCs werden toegewezen aan PDT met 5 -aminolevulinezuur (ALA-PDT, $n=84$ ) of chirurgische excisie (SE, $n=91$ ). ALA-PDT werd gefractioneerd uitgevoerd met twee gelijke belichtingen na resp. vier en vijf uur na de applicatie van ALA crème. In dit proefschrift worden de resultaten van een tussentijdse analyse met een follow-up tot 3 jaar beschreven. Incomplete respons of recidief werd gezien bij 17 BCCs, zestien maal na ALA-PDT en een maal na SE. Het ziekte-vrije percentage na 36 maanden, berekend middels Kaplan-Meier survivalanalyse, was met $66.5 \%$ voor PDT en $98.8 \%$ voor SE reeds statistisch significant $(p<0.001)$. Omdat uit de literatuur bekend is dat de meeste recidieven optreden na drie tot vijf jaar, is niet te verwachten dat de uitkomst van deze studie na een follow-up periode van vijf jaar ten gunste van ALA zal keren. Derhalve kan geconcludeerd worden dat gefractioneerde ALA-PDT minder geschikt is als reguliere behandeling voor $\mathrm{nBCC}$ dan SE. Echter, voor speciale gevallen zoals bij patiënten die niet geopereerd kunnen worden of basaalcel-naevus syndroom patiënten, zou er wel een plaats voor deze vorm van PDT mogelijk kunnen zijn. 


\section{Hoe pijnlijk is topicale fotodynamische therapie voor nodulair BCC?}

Pijn wordt vaak beschouwd als een belangrijke bijwerking van topicale PDT voor nietmelanoom huidkanker, vooral wanneer het behandeling van grote oppervlakten betreft. Deze pijn kan zelfs een limiterende factor zijn voor de behandeling. Het begrijpen van het mechanisme van deze pijn zou kunnen leiden tot oplossing van dit probleem wat weer zou kunnen leiden tot een betere toepasbaarheid van de therapie. Verdere studies om het mechanisme van pijn bij PDT te ontrafelen zijn dan ook nodig. Bij 64 nBCCs die behandeld werden met ALA-PDT, werden de intensiteit, duur en karakter van de pijn geanalyseerd. De gemiddelde pijnintensiteit, gemeten op een 'visual analogue scale' (VAS), bedroeg 3.88. De meeste patiënten kwalificeerden de pijn als brandend (82.5\%) of stekend $(36.8 \%)$. Behandeling van BCC met ALA-PDT resulteerde zelden in onhoudbare pijn en was nooit een reden om de behandeling te staken. Bij ALA-PDT met een tweetal metaalhalogeen lichtbronnen bleek belichting met de Medeikonos ${ }^{\circledR}$ lichtbron minder pijnlijk te zijn dan met de Waldmann ${ }^{\circledast}$ lichtbron. Vergelijking met nieuwere lichtbronnen, zoals met light emitting diodes (LED) is waardevol daar deze lampen omwille van praktische redenen steeds vaker worden gebruikt bij de uitvoering van PDT.

\section{Bestaat er een verschil tussen fotodynamische therapie met methylaminolevuline-} zuur en fotodynamische therapie met aminolevulinezuur in effectiviteit, pijnlijkheid en kosten?

Tegenwoordig worden binnen de dermatologie twee topicale fotosensitiserende stoffen veelvuldig toegepast, namelijk 5-aminolevulinezuur (ALA) en methylaminolevulinezuur (MAL). ALA wordt in de ziekenhuizen waar het wordt toegepast bereid tot een crème, MAL is als crème commercieel verkrijgbaar en is geregistreerd voor de behandeling van o.a. BCC. De effectiviteit, pijnbeleving en kosten van ALA-PDT en MAL-PDT werden vergeleken bij 43 nBCCs. Acht weken na behandeling werd het behandelde gebied geëxcideerd en histopathologisch onderzocht. In $28 \%$ van de gevallen werd residuaal tumorweefsel gevonden. Er werd geen verschil gevonden tussen ALA-PDT en MAL-PDT met betrekking tot effectiviteit en pijn. Echter, de kosten van MAL-PDT waren 6 maal hoger dan die van ALA-PDT. Er werd geconcludeerd dat zowel ALA als MAL kunnen worden toegepast als topicale fotosensibiliserende stof bij PDT voor BCC. De vraag of PDT een effectieve behandeling is voor deze indicatie, zal moeten blijken uit gerandomiseerde, gecontroleerde studies met lange follow-up duur. 


\section{Dankwoord}

$\mathrm{Nu}$ het wetenschappelijke gedeelte van mijn boekje gereed is, is de tijd aangebroken om alle mensen te bedanken die mij hebben geholpen met mijn promotie. Buiten dat dit het leukste onderdeel om te schrijven is, is het ook een gevaarlijk onderdeel: niemand mag vergeten worden!

Hier gaat ie dan, te beginnen met de belangrijke personen die van af het begin tot het eind bij mijn onderzoek betrokken zijn geweest.

Monique, mijn copromotor, jij bent voor mij de allerbelangrijkste persoon geweest in mijn promotietraject. Jij hebt me wegwijs gemaakt in de PDT en je hebt me Mohs' chirurgie geleerd. Met jouw ervaring heb je mij goed kunnen helpen met de opzet, uitvoering en met name met het analyseren van het onderzoek en het schrijven van de artikelen, ontzettend bedankt!

Professor Neumann, door menigeen al bejubeld, ik ben ontzettend blij dat ik $u$ heb leren kennen in Maastricht voordat $u$ naar Rotterdam vertrok, en dat u mij de mogelijkheid hebt gegeven om in dit interessante onderzoeksveld promotieonderzoek te doen. Mij iedere zes weken ontbieden op het 'bankje' om te praten over de voortgang van het onderzoek, was voor mij een goede stok achter de deur. Toen $u$ later naar Rotterdam vertrok bleef $u$ altijd beschikbaar om mij verder te helpen met het proefschrift, vaak op zondagochtend om 08.00 uur of feestdagen, in Banholt. Hartelijk bedankt voor uw promotorschap!

Professor Steijlen, toen $u$ in Maastricht arriveerde, werd u mijn opleider en mijn promotor. Niet iedereen heeft het geluk om door twee zo bijzondere hoogleraren begeleid te worden! Met uw weer andere, niet-Neumanniaanse kijk op onderzoek heeft $u$ mijn promotie een extra dimensie gegeven. Ik wil ook $u$ dan ook hartelijk bedanken voor uw interesse en begeleiding en voor het feit dat u mijn promotor wilde zijn.

Nicole, oncomaatje, congresmaatje, sportmaatje, kroegmaatje, maatje; bedankt dat jij een van mijn paranimfen wil zijn!

Adrienne, ik vind het een eer dat $\mathrm{jij}$ als goede vriendin en fantastische collega mijn paranimf wil zijn!

Anja, het is dat twee paranimfen gebruikelijk is, anders had ik graag jou ook nog gevraagd! Je bent een voorbeeld voor me door je professionele veelzijdigheid en wist me altijd moed in te praten als ik het even niet zag zitten. 
Gertruud en Judith, mijn dank dat ik in het laatste traject van mijn promotie de ruimte en tijd heb gekregen om bij jullie in het Catrien nog wat onderzoek kon afronden. Gertruud, ik ben vereerd door jouw deelname in de corona, leuk dat je me "het vuur aan de schenen" wil leggen. Je hebt een belangrijke rol gespeeld in de ontwikkeling en opzet van een aantal onderzoeken uit mijn boekje. Hopelijk kunnen we nog vele jaren de Brabantse dermatooncologie hoog houden!

Paul en Fons, jullie zijn heel belangrijk voor mij en mijn onderzoek geweest. In de tijd dat ik arts-assistent was bij jullie, hebben jullie met veel enthousiasme patiënten voor mij gerekruteerd en altijd veel interesse gehad in de voortgang van het onderzoek. De leuke tijd in jullie praktijk heeft mijn enthousiasme voor het vak alleen maar doen toenemen, bedankt voor deze tijd.

Patty, zonder jou was ik niet uit die lastige analyses gekomen. "Wanneer gebruik je nu welke test, kun je het nog één keer uitleggen?.." Bedankt voor je engelengeduld!

Brit, ontzettend bedankt voor al je vrije en nachtelijke uren die jij in de opmaak en het design van dit boekje hebt willen steken: het is echt super geworden, petje af!

Patricia, Roeland, Martijn, Martina, Carleine, Klara, Ruud, Sadhanna, Anne-moon, Jeroen, Jeroen, Yolande, Karin, Nadia, Heleen, Jaap, Esther, Valerie, Eugene en Ivo: jullie eindeloze interesse en medeleven was een stimulans voor mij. Ook dank voor jullie flexibiliteit in de tijd dat ik aan het schrijven was. Ik zal jullie gaan missen als ik uit Maastricht weg ben!

Een speciaal dankwoord wil ik richten aan Carleine die samen met mij de Metvix-ALA studie heeft uitgevoerd en aan Klara, die een groot deel van de PDT studie heeft gedaan. Klara, in Moniques boekje stond destijds dat ze het onderzoeksstokje aan mij doorgaf, bij deze hoop ik dat jij het promotieonderzoek naar BCC voortzet! Kim, jij natuurlijk ook bedankt voor de opzet en start van de transplantaten studie!

Patricia, jij in het bijzonder bedankt voor je steun, we zaten in het zelfde schuitje. Jij hebt het fantastisch gedaan, ik hoop in de buurt te komen.

Noor en Kirsten, jullie wetenschappelijke stage heeft al geresulteerd in een en binnenkort in twee publicaties. Dat is een fantastisch resultaat en het was ook erg gezellig met jullie! Annette, bedankt voor je inzet. 
Alle stafleden in Maastricht die nog niet de revue zijn gepasseerd dank ik hartelijk voor de interesse, steun en flexibiliteit. Ik ben trots om (inmiddels) jullie collega te zijn.

Kitty, bedankt voor de engelse correcties van een artikel. Bob Tank, bedankt voor hetzelfde bij een ander artikel. Angela, bedankt voor je kritische beoordeling van het radiotherapiestukje. Hopelijk feesten jullie mee!

Reno, bedankt voor het maken van de foto's van de histologische coupes!

Riëtte, ondanks dat ons onderzoek niet heeft geresulteerd in een artikel, toch bedankt voor gezellige samenwerking op al die dagen in jullie lab in Rotterdam.

Alle dames van het secretariaat, ontzettend bedankt voor jullie praktische en emotionele ondersteuning, jullie zijn schatten. Els extra bedankt voor het bijhouden van de indusies; Annelies extra bedankt voor het regelen van de promotie-perikelen.

Alle poli dames, en in het begin ook één heer, Frits, jullie ook bedankt voor de gezellige jaren en de flexibiliteit als ik weer patiënten tussendoor voor PDT ging plannen (en dan ook nog gefractioneerd).

Dan ben ik toe gekomen aan het bedanken van de belangrijkste mensen, en niet alleen in het promotietraject.

Pap en mam, zonder jullie was ik niet geworden wie ik nu ben. Jullie hebben altijd in mij geloofd en me onvoorwaardelijk gesteund, bedankt, bedankt. Simone, grote zus, je bent een voorbeeld voor mij met je hart voor patiënten en je harde werken, bedankt voor je niet aflatende interesse. Hopelijk gaan jullie dit met mij vieren tot in de kleine uurtjes!

Tenslotte Mark, iedereen die gepromoveerd is, weet hoe belangrijk het is dat je partner achter je staat. Jij hebt mij door dik en dun altijd gesteund en geholpen, ik kan je niet genoeg bedanken hiervoor. Lieve Teun, mama heeft nu in de weekeinden weer meer tijd om met papa en jou naar de geitjes te gaan!

Papa, mama en Mark: dit boekje is ook voor jullie! 


\section{List of publications}

Kuijpers DIM, Thissen MRTM, Neumann HAM. Basal cell carcinoma: treatment options and prognosis, a scientific approach to a common malignancy. Am J Clin Dermatol 2002;3(4):247-59.

Kuijpers DIM, Berretty PJM. Diagnose in beeld (102). Een man met jeuk aan de voet. Ned Tijdschr Geneeskd 2002;146:1585.

Kuijpers DIM. Agressief digitaal papillair adenocarcinoom. Ned Tijdschr Dermatol Venereol 2002;12:161-2.

Kuijpers DIM. Familiaire cilindromatosis: behandeling met de erbium-YAG en de CO2-laser. Ned Tijdschr Dermatol Venereol 2002;12:182-5.

Kuijpers DIM, Lücker GPH. Granuloma annulare disseminatum. Ned Tijdschr Dermatol Venereol 2003;13:280-3.

Kuijpers DI, Smeets NW, Krekels GA, Thissen MR. Photodynamic therapy as adjuvant treatment of extensive basal cell carcinoma treated with Mohs' micrographic surgery. Dermatol Surg 2004;30(5):794-8.

Smeets NW, Kuijpers DI, Nelemans P, et al. Mohs' micrographic surgery for treatment of basal cell carcinoma of the face--results of a retrospective study and review of the literature. Br J Dermatol 2004;151(1):141-7.

Thissen MR, Kuijpers DI, Neumann HA. Ruimere toepassing van fotodynamische therapie in de dermatologie. Ned Tijdschr Geneeskd 2005;149(5):232-7.

Oosten van EJ, Kuijpers DIM, Thissen MRTM. Different pain sensations in photodynamic therapy of nodular basal cell carcinoma. Results from a prospective trial and a review of the literature. Photodiagnosis and Photodynamic Therapy 2006;3:61-8.

Thissen MRTM, Kuijpers DIM. Local immune modulator (Imiquimod 5\% cream) as adjuvant treatment after incomplete Mohs' micrographic surgery for large, mixed type basal cell carcinoma. A report of 3 cases. J Drugs Dermatol 2006;5:461-4. 
Kuijpers DIM, Thissen MRTM, Thissen CA, Neumann HAM. Similar effectiveness of methyl aminolevulinate and 5-aminolevulinate in topical photodynamic therapy for nodular basal cell carcinoma. J Drugs Dermatol 2006;5:642-5

Kuijpers DIM, Hillen F, Frank J. Occupational peri-ocular contact dermatitis due to sensitization against black rubber components of a microscope. Contact Dermatitis 2006;55(2):7780.

Kuijpers DIM, Smeets NWJ, Lapière K, Thissen MRTM, Krekels GAM, Neumann HAM. Do systemic antibiotics increase the survival of a full thickness graft on the nose? Eur Acad Dermatol Venereol 2006;20(10):1296-301.

Kuijpers DIM, Kloos RJHM, Thissen MRTM. Merkelcelcarcinoom. Ned Tijdschr Dermatol Venereol 2006;16:202-4.

Kuijpers DI, Thissen MR, Berretty PJ, Ideler AH, Nelemans P, Neumann HA. Surgical excision vs. curettage plus cryosurgery versus in the treatment of basal cell carcinoma in the head and neck area. Results from a randomised controlled trial. Accepted Dermatol Surg. 


\section{Curriculum Vitae}

Daniëlle Irene Maria Kuijpers werd op 22 oktober 1971 geboren in Tilburg. Ze groeide op in Eindhoven en Veldhoven, waar ze in 1990 het VWO diploma behaalde aan het Anton van Duinkerken College. Hierop volgde een jaar gezondheidswetenschappen aan de Rijksuniversiteit Limburg in Maastricht dat resulteerde in een propedeuse. In 1991 startte ze met de studie Geneeskunde eveneens aan de Rijksuniversiteit Limburg. Tijdens de studie heeft zij haar wetenschappelijke stage gedaan in Togo en in Uganda. In 1998 behaalde zij het artsexamen. De interesse voor het vak dermatologie was inmiddels sterk gegroeid. Om algemene ervaring op te doen werkte zij van april 1998 tot juli 1999 als AGNIO algemene heelkunde in het Maasland ziekenhuis te Sittard. Hierna kon zij aan de slag als AGNIO dermatologie in Maastricht, destijds nog bij prof. Neumann. Na een leerzame periode van twee jaar als AGNIO in het Catharina ziekenhuis te Eindhoven bij dr. Berretty en dr. Ideler, begon zij in januari 2002 aan de opleiding tot dermatoloog in het academisch ziekenhuis Maastricht, nu bij prof. Steijlen. De opleiding zal op 1 januari 2007 worden afgerond. 